\title{
A Pd-Catalyzed Site-Controlled Isomerization of Terminal Olefins
}

\author{
Wenlong Ren, ${ }^{\text {a\# }}$ Fei Sun, ${ }^{\text {a\# }}$ Jianxiao Chu, ${ }^{\text {a }}$ and Yian Shi*,a,b
}

${ }^{a}$ Institute of Natural and Synthetic Organic Chemistry, Changzhou University, Changzhou 213164, China;

${ }^{b}$ Department of Chemistry, Colorado State University, Fort Collins, Colorado 80523, United States.

\section{Supporting Information}

\section{Table of Contents}

Table S1

Table S2

Table S3

Experimental procedures

S-6

Characterization data

S-6

NMR spectra

S-16 
Table S1. Studies of the Reaction Conditions ${ }^{a}$

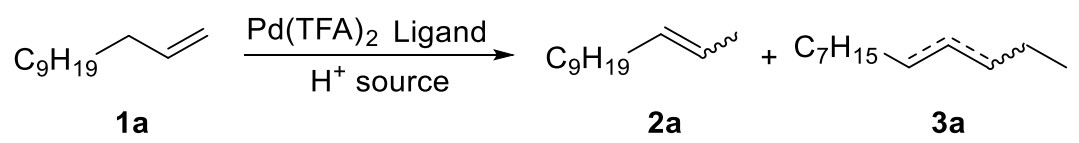

\begin{tabular}{|c|c|c|c|c|c|}
\hline entry & ligand & $\mathrm{H}^{+}$source & conv. $(\%)^{b}$ & $\mathbf{2} \mathbf{a}(\%)(\mathrm{E} / \mathrm{Z})^{b}$ & $\mathbf{3 a}(\%)^{b}$ \\
\hline 1 & $\mathbf{L 1}$ & $\mathrm{PhOH}$ & 44 & $44(13: 1)$ & trace \\
\hline 2 & $\mathrm{PPh}_{3}$ & $\mathrm{PhOH}$ & trace & -- & -- \\
\hline 3 & $\mathbf{L} 2$ & $\mathrm{PhOH}$ & 0 & -- & -- \\
\hline 4 & $\mathbf{L 3}$ & $\mathrm{PhOH}$ & trace & -- & -- \\
\hline 5 & L4 & $\mathrm{PhOH}$ & 17 & $15(2: 3)$ & 2 \\
\hline 6 & dppe & $\mathrm{PhOH}$ & trace & -- & -- \\
\hline 7 & dppp & $\mathrm{PhOH}$ & 0 & -- & -- \\
\hline 8 & $\mathrm{dppb}$ & $\mathrm{PhOH}$ & 0 & -- & -- \\
\hline 9 & xantphos & $\mathrm{PhOH}$ & 20 & $12(1: 1)$ & 8 \\
\hline 10 & BINAP & $\mathrm{PhOH}$ & trace & -- & -- \\
\hline $11^{c}$ & L1 & $\mathrm{PhOH}$ & 0 & -- & -- \\
\hline $12^{d}$ & $\mathbf{L 1}$ & $\mathrm{PhOH}$ & 0 & -- & -- \\
\hline 13 & $\mathbf{L 1}$ & $p-\mathrm{ClPhOH}$ & 59 & $58(13: 1)$ & 1 \\
\hline 14 & L1 & $\mathrm{F}_{5} \mathrm{PhOH}$ & 99 & $87(10: 1)$ & 12 \\
\hline 15 & L1 & $\mathrm{MeOH}$ & 12 & $12(10: 1)$ & -- \\
\hline 16 & $\mathbf{L 1}$ & $\mathrm{EtOH}$ & trace & -- & -- \\
\hline $17^{e}$ & L1 & $\mathrm{FCH}_{2} \mathrm{CH}_{2} \mathrm{OH}$ & 94 & $91(13: 1)$ & 3 \\
\hline 18 & $\mathbf{L 1}$ & $\mathrm{F}_{2} \mathrm{CHCH}_{2} \mathrm{OH}$ & 97 & $89(11: 1)$ & 8 \\
\hline 19 & $\mathbf{L 1}$ & $\mathrm{F}_{3} \mathrm{CCH}_{2} \mathrm{OH}$ & 75 & $74(13: 1)$ & 1 \\
\hline 20 & L1 & $\mathrm{HFIP}^{l}$ & 18 & $18(10: 1)$ & - \\
\hline 21 & L1 & $\mathrm{HCOOH}$ & 87 & $85(13: 1)$ & 2 \\
\hline 22 & L1 & $\mathrm{AcOH}$ & 80 & $79(13: 1)$ & 1 \\
\hline 23 & L1 & TFA & 99 & $80(5: 1)$ & 19 \\
\hline 24 & L1 & $\mathrm{TsOH} \cdot \mathrm{H}_{2} \mathrm{O}$ & 37 & $36(5: 1)$ & 1 \\
\hline $25^{f}$ & $\mathbf{L 1}$ & $\mathrm{FCH}_{2} \mathrm{CH}_{2} \mathrm{OH}$ & 94 & $91(13: 1)$ & 3 \\
\hline $26^{g}$ & $\mathbf{L 1}$ & $\mathrm{FCH}_{2} \mathrm{CH}_{2} \mathrm{OH}$ & 12 & $12(12: 1)$ & -- \\
\hline $27^{h}$ & L1 & $\mathrm{FCH}_{2} \mathrm{CH}_{2} \mathrm{OH}$ & trace & -- & -- \\
\hline $28^{i}$ & L1 & $\mathrm{FCH}_{2} \mathrm{CH}_{2} \mathrm{OH}$ & 0 & -- & -- \\
\hline $29^{j}$ & L1 & $\mathrm{FCH}_{2} \mathrm{CH}_{2} \mathrm{OH}$ & 97 & $89(10: 1)$ & 8 \\
\hline $30^{k}$ & $\mathbf{L 1}$ & $\mathrm{FCH}_{2} \mathrm{CH}_{2} \mathrm{OH}$ & 97 & $82(8: 1)$ & 15 \\
\hline
\end{tabular}


${ }^{a}$ The reactions were carried out with $1 \mathrm{a}(0.50 \mathrm{mmol}), \operatorname{Pd}(\mathrm{TFA})_{2}(0.025 \mathrm{mmol})$, ligand $(0.050$ or $0.10 \mathrm{mmol}, \mathrm{P} / \mathrm{Pd}=4: 1)$, and $\mathrm{H}^{+}$source $(0.25 \mathrm{mmol})$ in DCE $(0.30 \mathrm{~mL})$ at $\mathrm{rt}\left(25-27{ }^{\circ} \mathrm{C}\right)$ for 24 $\mathrm{h}$ unless otherwise stated. ${ }^{b}$ The conversion was determined by the ${ }^{1} \mathrm{H}$ NMR analysis of the crude reaction mixture with BnOMe as internal standard and also confirmed by the ${ }^{1} \mathrm{H}$ NMR analysis of isolated products. The content of $\mathbf{2 a}$ and $\mathbf{3 a}$ as well as the E/Z ratio of $\mathbf{2 a}$ were determined by ${ }^{1} \mathrm{H}$ NMR analysis of isolated products (ref. 13). ${ }^{c}$ With $\mathrm{Pd}(\mathrm{OAc})_{2}(0.025 \mathrm{mmol}) .{ }^{d}$ With $\mathrm{Pd}(\mathrm{dba})_{2}(0.025 \mathrm{mmol}) .{ }^{e} 33 \%$ conversion obtained with $0.10 \mathrm{mmol} \mathrm{FCH}_{2} \mathrm{CH}_{2} \mathrm{OH} .{ }^{f}$ With DCM. ${ }^{g}$ With toluene. ${ }^{h}$ With THF. ${ }^{i}$ At $0{ }^{\circ} \mathrm{C} .{ }^{j}$ At $60{ }^{\circ} \mathrm{C} .{ }^{k}$ At $80{ }^{\circ} \mathrm{C} .{ }^{l} \mathrm{HFIP}=$ hexafluoroisopropanol.

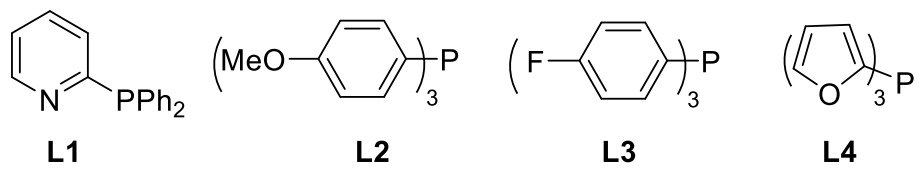


Table S2. Studies of Ligands ${ }^{a}$

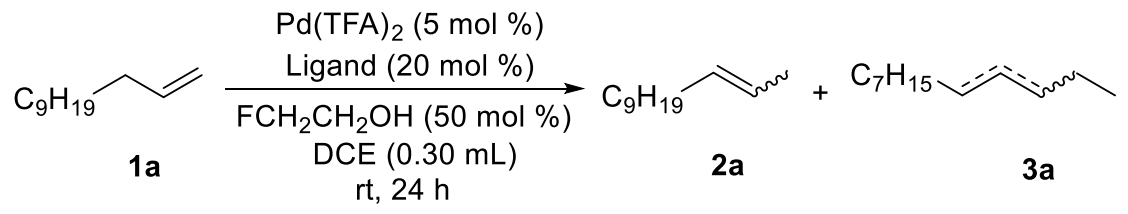

$\begin{array}{lllll}\text { entry } & \text { ligand } & \text { conv. }(\%)^{b} & \mathbf{2 a}(\%)(\mathrm{E} / \mathrm{Z})^{b} & \mathbf{3 a}(\%)^{b} \\ 1 & \text { L1 } & 94 & 91(13: 1) & 3 \\ 2 & \text { L5 } & \text { trace } & - & - \\ 3 & \text { L6 } & \text { trace } & - & - \\ 4 & \text { L7 } & 97 & 74(10: 1) & 23 \\ 5 & \text { L8 } & 100 & 61(4: 1) & 39 \\ 6 & \text { L9 } & 96 & 84(10: 1) & 12 \\ 7 & \text { L10 } & 94 & 88(12: 1) & 6\end{array}$

${ }^{a}$ The reactions were carried out with substrate $1 \mathbf{a}(0.50 \mathrm{mmol}), \mathrm{Pd}(\mathrm{TFA})_{2}(0.025 \mathrm{mmol})$, ligand $(0.10 \mathrm{mmol})$, and $\mathrm{FCH}_{2} \mathrm{CH}_{2} \mathrm{OH}(0.25 \mathrm{mmol})$ in DCE $(0.30 \mathrm{~mL})$ at $\mathrm{rt}$ for $24 \mathrm{~h}$ unless otherwise stated. ${ }^{b}$ The conversion was determined by the ${ }^{1} \mathrm{H}$ NMR analysis of the crude reaction mixture with BnOMe as internal standard. The content of $\mathbf{2 a}$ and $\mathbf{3 a}$ as well as the $\mathrm{E} / \mathrm{Z}$ ratio of $\mathbf{2 a}$ were also determined by ${ }^{1} \mathrm{H}$ NMR analysis of crude reaction mixture

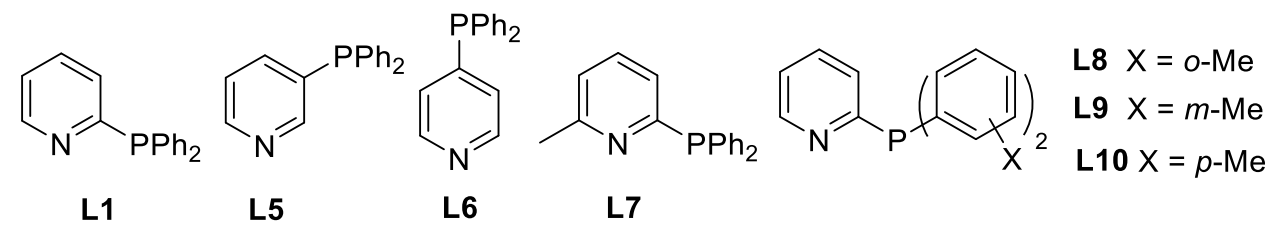


Table S3. Studies of Reaction Parameters ${ }^{a}$

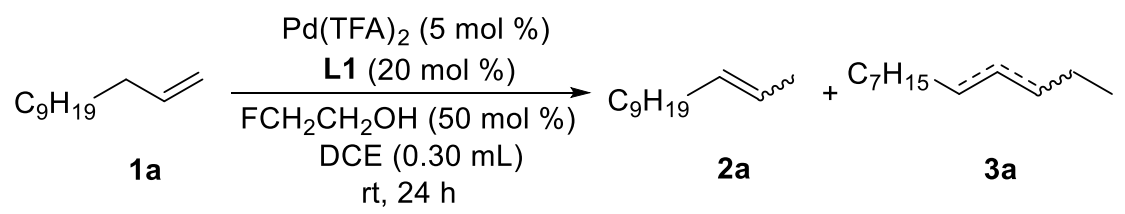

\begin{tabular}{|c|c|c|c|c|}
\hline entry & conditions & conv. $(\%)^{b}$ & $\mathbf{2 a}(\%)(\mathrm{E} / \mathrm{Z})^{b}$ & $\mathbf{3 a}(\%)^{b}$ \\
\hline 1 & standard condition & 94 & $91(13: 1)$ & 3 \\
\hline 2 & $\mathrm{Pd} / \mathrm{P}=1: 1$ & 0 & - & - \\
\hline 3 & $\mathrm{Pd} / \mathrm{P}=1: 2$ & trace & - & - \\
\hline 4 & $\mathrm{Pd} / \mathrm{P}=1: 3$ & 78 & $77(13: 1)$ & 1 \\
\hline 5 & $\mathbf{L} \mathbf{1}+\mathrm{FCH}_{2} \mathrm{CH}_{2} \mathrm{OH}$ & 0 & - & - \\
\hline 6 & $\operatorname{Pd}(\mathrm{TFA})_{2}+\mathbf{L} \mathbf{1}$ & 0 & - & - \\
\hline 7 & $\mathrm{Pd}(\mathrm{TFA})_{2}+\mathrm{FCH}_{2} \mathrm{CH}_{2} \mathrm{OH}$ & 12 & $9(2: 1)$ & 3 \\
\hline 8 & $\operatorname{Pd}(\mathrm{TFA})_{2}$ & 11 & $11(2: 1)$ & trace \\
\hline 9 & $\operatorname{Pd}(\mathrm{TFA})_{2}+\mathrm{TFA}(5 \mathrm{~mol} \%)$ & 53 & $45(3: 1)$ & 8 \\
\hline 10 & $\mathrm{Pd}(\mathrm{TFA})_{2}+\mathrm{TFA}(10 \mathrm{~mol} \%)$ & 94 & $64(3: 1)$ & 30 \\
\hline 11 & $\mathrm{Pd}(\mathrm{TFA})_{2}+\mathrm{TFA}(20 \mathrm{~mol} \%)$ & 100 & $29(3: 1)$ & 71 \\
\hline 12 & TFA $(50 \mathrm{~mol} \%)$ & trace & - & - \\
\hline 13 & $\mathrm{PdCl}_{2}+\mathbf{L} \mathbf{1}+\mathrm{FCH}_{2} \mathrm{CH}_{2} \mathrm{OH}$ & 0 & - & - \\
\hline 14 & $\mathrm{Pd}(\mathrm{OAc})_{2}+\mathbf{L} \mathbf{1}+\mathrm{FCH}_{2} \mathrm{CH}_{2} \mathrm{OH}$ & trace & - & - \\
\hline 15 & $\mathrm{Pd}\left(\mathrm{NO}_{3}\right)_{2}+\mathbf{L} \mathbf{1}+\mathrm{FCH}_{2} \mathrm{CH}_{2} \mathrm{OH}$ & 94 & $88(13: 1)$ & 6 \\
\hline 16 & $\mathrm{Pd}(\mathrm{dba})_{2}+\mathbf{L} \mathbf{1}+\mathrm{FCH}_{2} \mathrm{CH}_{2} \mathrm{OH}$ & 0 & - & - \\
\hline 17 & $\mathrm{Pd}(\mathrm{dba})_{2}+\mathrm{FCH}_{2} \mathrm{CH}_{2} \mathrm{OH}$ & 57 & $47(2: 1)$ & 10 \\
\hline 18 & $\operatorname{Pd}(\mathrm{dba})_{2}+\mathbf{L 1}+\mathrm{TFA}(10 \mathrm{~mol} \%)$ & 92 & $89(12: 1)$ & 3 \\
\hline 19 & $\mathrm{Pd}(\mathrm{dba})_{2}+\mathrm{TFA}(10 \mathrm{~mol} \%)$ & 56 & $51(2: 1)$ & 5 \\
\hline \multicolumn{5}{|c|}{$\begin{array}{l}{ }^{a} \text { The reactions were carried out with } 1(0.50 \mathrm{mmol}), \mathrm{Pd}(\mathrm{TFA})_{2}(0.025 \mathrm{mmol}) \text {, ligand }(0.10 \mathrm{mmol} \text {, } \\
\mathrm{P} / \mathrm{Pd}=4: 1) \text {, and } \mathrm{FCH}_{2} \mathrm{CH}_{2} \mathrm{OH}(0.25 \mathrm{mmol}) \text { in } \mathrm{DCE}(0.30 \mathrm{~mL}) \text { at } \mathrm{rt} \text { for } 24 \mathrm{~h} \text { unless otherwise } \\
\text { stated. }{ }^{b} \text { The conversion was determined by the }{ }^{1} \mathrm{H} \text { NMR analysis of the crude reaction mixture } \\
\text { with BnOMe as internal standard. The content of } \mathbf{2 a} \text { and } \mathbf{3 a} \text { as well as the } \mathrm{E} / \mathrm{Z} \text { ratio of } \mathbf{2 a} \text { were } \\
\text { also determined by }{ }^{1} \mathrm{H} \text { NMR analysis of crude reaction mixture. }\end{array}$} \\
\hline
\end{tabular}


General Methods. All commercially available reagents were used without further purification unless otherwise noted. All solvents used for the reaction were purified with solvent purification system. Column chromatography was performed on silica gel (300-400 mesh). $\quad{ }^{1} \mathrm{H}$ NMR spectra were recorded on a $400 \mathrm{MHz}$ NMR spectrometer, and ${ }^{13} \mathrm{C}$ NMR spectra were recorded on a $100 \mathrm{MHz}$ NMR spectrometer. IR spectra were recorded on a FTIR spectrometer. Melting points were uncorrected. Ligands L5, L6, and L8-L10 were prepared according to the reported procedures. ${ }^{1,2}$

1. Ponsico, S.; Gulyas, H.; Martínez-Belmonte, M.; Escudero-Adán, E.C.; Freixa, Z.; van Leeuwen, P.W.N.M. Dalton Trans. 2011, 40, 10686.

2. Li, Y.; Das, S.; Zhou, S.; Junge, K.; Beller, M. J. Am. Chem. Soc. 2012, 134, 9727.

\section{Representative procedure for isomerization of olefin (Table 2, entry 1)}

To a mixture of Pd(TFA $)_{2}(0.0083 \mathrm{~g}, 0.025 \mathrm{mmol})$ and ligand $\mathbf{L 1}(0.0263 \mathrm{~g}, 0.10 \mathrm{mmol})$ in a vial $(2.0 \mathrm{~mL})$ were added 2-fluoroethanol (0.016 g, $0.25 \mathrm{mmol})$, 1-dodecene (1a) $(0.0842$ g, $0.50 \mathrm{mmol})$, and dry DCE $(0.30 \mathrm{~mL})$ successively via syringe. The vial was purged with Ar to remove the air and tightly sealed with a septum cap. The reaction mixture was stirred at room temperature for $24 \mathrm{~h}$ and purified by flash chromatography (silica gel, eluent: petroleum ether) to give olefin $\mathbf{2 a}$ as a colorless oil (0.0798 g, 95\% yield).

\section{Representative procedure for isomerization of olefin (Table 2, entry 19)}

To a mixture of Pd(TFA $)_{2}(0.0083 \mathrm{~g}, 0.025 \mathrm{mmol})$ and ligand L1 $(0.0263 \mathrm{~g}, 0.10 \mathrm{mmol})$ in a vial $(2.0 \mathrm{~mL})$ were added (4-methylenecyclohexyl)benzene (1s) $(0.0861 \mathrm{~g}, 0.50 \mathrm{mmol})$, $\mathrm{F}_{5} \mathrm{PhOH}(0.092 \mathrm{~g}, 0.50 \mathrm{mmol})$, and dry DCE $(0.30 \mathrm{~mL})$ successively via syringe. The vial was purged with Ar to remove the air and tightly sealed with a septum cap. The reaction mixture was stirred at $50{ }^{\circ} \mathrm{C}$ in oil bath for $24 \mathrm{~h}$ and purified by flash chromatography (silica gel, eluent: petroleum ether) to give olefin $2 \mathrm{~s}$ as a light yellow oil ( $0.0822 \mathrm{~g}, 95 \%$ yield).

\section{Table 2, entry 1}

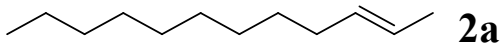

Colorless oil; $0.0798 \mathrm{~g}(95 \%) ; \quad$ IR (film) $1640 \mathrm{~cm}^{-1} ; \quad{ }^{1} \mathrm{H}$ NMR $\left(400 \mathrm{MHz}, \mathrm{C}_{6} \mathrm{D}_{6}\right) \delta 5.53-$ 
$5.37(\mathrm{~m}, 2 \mathrm{H}), 2.08-1.96(\mathrm{~m}, 2 \mathrm{H}), 1.62(\mathrm{~d}, J=4.8 \mathrm{~Hz}, 3 \mathrm{H}), 1.42-1.22(\mathrm{~m}, 14 \mathrm{H}), 0.91(\mathrm{t}, J=6.6$ $\mathrm{Hz}, 3 \mathrm{H}) ; \quad{ }^{13} \mathrm{C} \mathrm{NMR}\left(100 \mathrm{MHz}, \mathrm{C}_{6} \mathrm{D}_{6}\right) \delta 132.0,124.9,33.2,32.4,30.14,30.13,30.1,29.9,29.7$, 23.2, 18.2, 14.4; HRMS (ESI) Calcd for $\mathrm{C}_{12} \mathrm{H}_{28} \mathrm{~N}\left[\mathrm{M}+\mathrm{NH}_{4}\right]^{+}:$186.2216; Found: 186.2217.

Bissember, A.C.; Levina, A.; Fu, G. C. J. Am. Chem. Soc. 2012, 134, 14232.

\section{Table 2, entry 2}

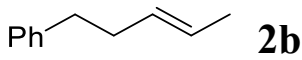

Colorless oil; 0.0656 g (90\%); IR (film) $1646 \mathrm{~cm}^{-1} ; \quad{ }^{1} \mathrm{H}$ NMR (400 MHz, $\left.\mathrm{C}_{6} \mathrm{D}_{6}\right) \delta 7.20-$ $7.13(\mathrm{~m}, 2 \mathrm{H}), 7.10-7.02(\mathrm{~m}, 3 \mathrm{H}), 5.47-5.30(\mathrm{~m}, 2 \mathrm{H}), 2.59-2.52(\mathrm{~m}, 2 \mathrm{H}), 2.31-2.19(\mathrm{~m}, 2 \mathrm{H}), 1.55$ $(\mathrm{d}, J=4.8 \mathrm{~Hz}, 3 \mathrm{H}) ; \quad{ }^{13} \mathrm{C} \mathrm{NMR}\left(100 \mathrm{MHz}, \mathrm{C}_{6} \mathrm{D}_{6}\right) \delta 142.4,131.0,128.8,128.6,126.1,125.5$, 36.5, 34.9, 18.1; HRMS (EI) Calcd for $\mathrm{C}_{11} \mathrm{H}_{14}[\mathrm{M}]^{+}: \quad$ 146.1090; Found: 146.1091 . Wang, Y.; Qin, C.; Jia, X.; Leng, X.; Huang, Z. Angew. Chem. Int. Ed. 2017, 56, 1614.

\section{Table 2, entry 3}

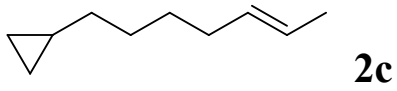

Colorless oil; $0.0618 \mathrm{~g}(89 \%)$; IR (film) $1640 \mathrm{~cm}^{-1} ; \quad{ }^{1} \mathrm{H}$ NMR (400 MHz, $\left.\mathrm{C}_{6} \mathrm{D}_{6}\right) \delta 5.52-$ $5.36(\mathrm{~m}, 2 \mathrm{H}), 2.07-1.94(\mathrm{~m}, 2 \mathrm{H}), 1.62$ (d, $J=4.8 \mathrm{~Hz}, 3 \mathrm{H}), 1.44-1.30$ (m, 4H), 1.20-1.09 (m, 2H), 0.64-0.52 (m, 1H), 0.43-0.32 (m, 2H), 0.02-(-0.04) (m, 2H); ${ }^{13} \mathrm{C}$ NMR (100 MHz, $\left.\mathrm{C}_{6} \mathrm{D}_{6}\right)$ $\delta 132.0,124.9,35.1,33.2,29.9,29.6,18.1,11.3,4.9 ; \quad$ HRMS (ESI) Calcd for $\mathrm{C}_{10} \mathrm{H}_{18} \mathrm{~K}[\mathrm{M}+\mathrm{K}]^{+}$: 177.1040; Found: 177.1045.

\section{Table 2, entry 4}

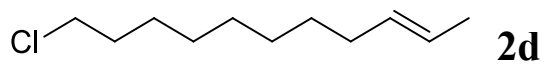

Colorless oil; $0.0894 \mathrm{~g}(95 \%)$; IR (film) $1642 \mathrm{~cm}^{-1} ; \quad{ }^{1} \mathrm{H}$ NMR (400 MHz, $\left.\mathrm{C}_{6} \mathrm{D}_{6}\right) \delta 5.54-$ $5.38(\mathrm{~m}, 2 \mathrm{H}), 3.12(\mathrm{t}, J=6.7 \mathrm{~Hz}, 2 \mathrm{H}), 2.06-1.95(\mathrm{~m}, 2 \mathrm{H}), 1.63(\mathrm{~d}, J=4.5 \mathrm{~Hz}, 3 \mathrm{H}), 1.48-1.39$ $(\mathrm{m}, 2 \mathrm{H}), 1.37-1.27(\mathrm{~m}, 2 \mathrm{H}), 1.25-1.01(\mathrm{~m}, 8 \mathrm{H}) ; \quad{ }^{13} \mathrm{C} \mathrm{NMR}\left(100 \mathrm{MHz}, \mathrm{C}_{6} \mathrm{D}_{6}\right) \delta 131.9,124.9$, 45.0, 33.1, 32.9, 30.0, 29.7, 29.5, 29.2, 27.1, 18.2; $\quad$ HRMS (ESI) Calcd for $\mathrm{C}_{11} \mathrm{H}_{20} \mathrm{Cl}[\mathrm{M}-\mathrm{H}]^{-}$: 
187.1259; Found: 187.1259.

Wang, Y.; Qin, C.; Jia, X.; Leng, X.; Huang, Z. Angew. Chem. Int. Ed. 2017, 56, 1614.

\section{Table 2, entry 5}

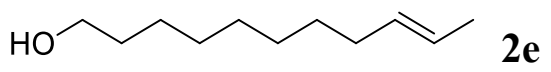

Colorless oil; $\quad 0.0817 \mathrm{~g}$ (96\%); $\quad$ IR (film) 3337, $1658 \mathrm{~cm}^{-1} ;{ }^{1} \mathrm{H}$ NMR (400 MHz, $\left.\mathrm{C}_{6} \mathrm{D}_{6}\right) \delta$ 5.52-5.38 (m, 2H), $3.37(\mathrm{t}, J=6.4 \mathrm{~Hz}, 2 \mathrm{H}), 2.08-1.97(\mathrm{~m}, 2 \mathrm{H}), 1.62(\mathrm{~d}, J=4.7 \mathrm{~Hz}, 3 \mathrm{H}), 1.43-$ $1.18(\mathrm{~m}, 12 \mathrm{H}), 0.88(\mathrm{~s}, 1 \mathrm{H}) ; \quad{ }^{13} \mathrm{C} \mathrm{NMR}\left(100 \mathrm{MHz}, \mathrm{C}_{6} \mathrm{D}_{6}\right) \delta 132.0,124.9,62.7,33.2,33.1,30.1$, 30.0, 29.9, 29.6, 26.3, 18.1; HRMS (ESI) Calcd for $\mathrm{C}_{11} \mathrm{H}_{23} \mathrm{O}[\mathrm{M}+\mathrm{H}]^{+}: \quad$ 171.1743; Found: 171.1742 .

a) Runge, M.B.; Mwangi, M.T.; Bowden, N.B. J. Organomet. Chem. 2006, 691, 5278.

b) Wang, Y.; Qin, C.; Jia, X.; Leng, X.; Huang, Z. Angew. Chem. Int. Ed. 2017, 56, 1614.

\section{Table 2, entry 6}

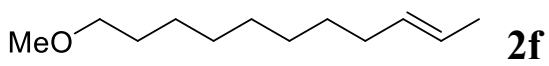

Colorless oil; $0.0866 \mathrm{~g}$ (94\%); IR (film) $1642 \mathrm{~cm}^{-1} ; \quad{ }^{1} \mathrm{H}$ NMR (400 MHz, $\left.\mathrm{C}_{6} \mathrm{D}_{6}\right) \delta 5.54-$ $5.37(\mathrm{~m}, 2 \mathrm{H}), 3.22(\mathrm{t}, J=6.4 \mathrm{~Hz}, 2 \mathrm{H}), 3.15(\mathrm{~s}, 3 \mathrm{H}), 2.07-1.96(\mathrm{~m}, 2 \mathrm{H}), 1.62(\mathrm{~d}, J=4.6 \mathrm{~Hz}, 3 \mathrm{H})$, 1.61-1.52(m, 2H), 1.40-1.20 (m, 10H); $\left.{ }^{13} \mathrm{C} \mathrm{NMR} \mathrm{(100} \mathrm{MHz,} \mathrm{C}_{6} \mathrm{D}_{6}\right) \delta$ 131.8, 124.6, 72.7, 58.1, 32.9, 30.0, 29.8, 29.74, 29.7, 29.4, 26.4, 17.9; HRMS (ESI) Calcd for $\mathrm{C}_{12} \mathrm{H}_{25} \mathrm{O}[\mathrm{M}+\mathrm{H}]^{+}$: 185.1900; Found: 185.1899.

\section{Table 2, entry 7}

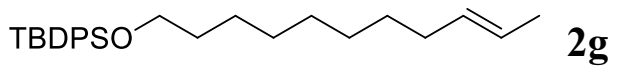

Colorless oil; $0.1998 \mathrm{~g}$ (98\%); IR (film) $1654 \mathrm{~cm}^{-1} ; \quad{ }^{1} \mathrm{H}$ NMR (400 MHz, $\left.\mathrm{C}_{6} \mathrm{D}_{6}\right) \delta 7.84-$ $7.78(\mathrm{~m}, 4 \mathrm{H}), 7.27-7.22(\mathrm{~m}, 6 \mathrm{H}), 5.52-5.38(\mathrm{~m}, 2 \mathrm{H}), 3.69(\mathrm{t}, J=6.4 \mathrm{~Hz}, 2 \mathrm{H}), 2.06-1.96(\mathrm{~m}, 2 \mathrm{H})$, $1.63(\mathrm{~d}, J=4.8 \mathrm{~Hz}, 3 \mathrm{H}), 1.61-1.54(\mathrm{~m}, 2 \mathrm{H}), 1.40-1.16(\mathrm{~m}, 10 \mathrm{H}), 1.20(\mathrm{~s}, 9 \mathrm{H}) ; \quad{ }^{13} \mathrm{C} \mathrm{NMR}(100$ $\left.\mathrm{MHz}, \mathrm{C}_{6} \mathrm{D}_{6}\right) \delta 136.0,134.5,132.0,129.9,128.1,124.8,64.3,33.13,33.1,30.1,29.9,29.8,29.6$, 
27.2, 26.2, 19.5, 18.2; HRMS (ESI) Calcd for $\mathrm{C}_{27} \mathrm{H}_{41} \mathrm{OSi}[\mathrm{M}+\mathrm{H}]^{+}$: 409.2921; Found: 409.2918 .

\section{Table 2, entry 8}

$\mathrm{OHC}$

\section{$2 \mathrm{~h}$}

Colorless oil; $\quad 0.0797 \mathrm{~g}(95 \%)$; IR (film) 1727, $1640 \mathrm{~cm}^{-1} ;{ }^{1} \mathrm{H}$ NMR (400 MHz, $\left.\mathrm{C}_{6} \mathrm{D}_{6}\right) \delta$ $9.32(\mathrm{t}, J=1.9 \mathrm{~Hz}, 1 \mathrm{H}), 5.54-5.38(\mathrm{~m}, 2 \mathrm{H}), 2.06-1.94(\mathrm{~m}, 2 \mathrm{H}), 1.80(\mathrm{td}, J=7.3,1.5 \mathrm{~Hz}, 2 \mathrm{H})$, $1.63(\mathrm{~d}, J=4.0 \mathrm{~Hz}, 3 \mathrm{H}), 1.34-1.24(\mathrm{~m}, 4 \mathrm{H}), 1.22-0.98(\mathrm{~m}, 6 \mathrm{H}) ;{ }^{13} \mathrm{C} \mathrm{NMR}\left(100 \mathrm{MHz}, \mathrm{C}_{6} \mathrm{D}_{6}\right)$ $\delta 200.8,131.9,124.9,43.8,33.1,29.9,29.6,29.4,29.3,22.2,18.1$; HRMS (ESI) Calcd for $\mathrm{C}_{11} \mathrm{H}_{19} \mathrm{O}[\mathrm{M}-\mathrm{H}]^{-}: \quad 167.1441 ;$ Found: 167.1437.

\section{Table 2, entry 9}

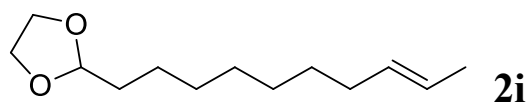

Light yellow oil; $\quad 0.1014 \mathrm{~g}(95 \%) ; \quad$ IR (film) $1640 \mathrm{~cm}^{-1} ; \quad{ }^{1} \mathrm{H}$ NMR $\left(400 \mathrm{MHz}, \mathrm{C}_{6} \mathrm{D}_{6}\right) \delta 5.50-$ $5.36(\mathrm{~m}, 2 \mathrm{H}), 4.84(\mathrm{t}, J=4.8 \mathrm{~Hz}, 1 \mathrm{H}), 3.60-3.50(\mathrm{~m}, 2 \mathrm{H}), 3.44-3.34(\mathrm{~m}, 2 \mathrm{H}), 2.05-1.93(\mathrm{~m}, 2 \mathrm{H})$, 1.81-1.74 (m, 2H), $1.62(\mathrm{~d}, J=4.7 \mathrm{~Hz}, 3 \mathrm{H}), 1.57-1.47(\mathrm{~m}, 2 \mathrm{H}), 1.37-1.17(\mathrm{~m}, 8 \mathrm{H}) ; \quad{ }^{13} \mathrm{C} \mathrm{NMR}$ $\left(100 \mathrm{MHz}, \mathrm{C}_{6} \mathrm{D}_{6}\right) \delta 132.0,124.8,105.0,64.9,34.6,33.1,30.02,30.0,29.9,29.5,24.6,18.1$; HRMS (ESI) Calcd for $\mathrm{C}_{13} \mathrm{H}_{24} \mathrm{NaO}_{2}[\mathrm{M}+\mathrm{Na}]^{+}$: 235.1669; Found: 235.1665.

\section{Table 2, entry 10}

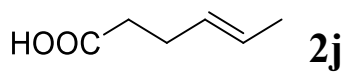

Light yellow oil; $\quad 0.0550 \mathrm{~g}(96 \%) ; \quad$ IR (film) 1711, $1642 \mathrm{~cm}^{-1} ; \quad{ }^{1} \mathrm{H}$ NMR (400 MHz, $\left.\mathrm{C}_{6} \mathrm{D}_{6}\right)$

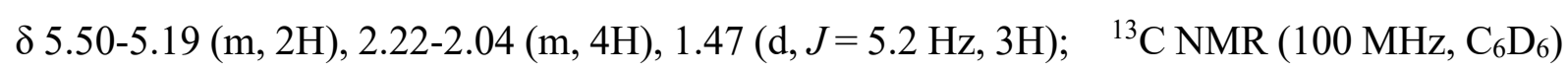
$\delta 180.5,129.3,126.4,34.2,27.8,17.9 ; \quad$ HRMS (ESI) Calcd for $\mathrm{C}_{6} \mathrm{H}_{9} \mathrm{O}_{2}[\mathrm{M}-\mathrm{H}]^{-}: \quad 113.0608$; Found: 113.0605 .

Blenderman, W.G.; Joullié, M.M. J. Org. Chem. 1983, 48, 3206. 


\section{Table 2, entry 11}

EtOOC $2 k$

Colorless oil; $\quad 0.1163 \mathrm{~g}(98 \%)$;R (film) 1722, $1655 \mathrm{~cm}^{-1} ; \quad{ }^{1} \mathrm{H}$ NMR (400 MHz, $\left.\mathrm{C}_{6} \mathrm{D}_{6}\right) \delta$ 7.07 (dt, $J=15.6,7.0 \mathrm{~Hz}, 1 \mathrm{H}), 5.90$ (d, $J=15.6 \mathrm{~Hz}, 1 \mathrm{H}), 5.53-5.38$ (m, 2H), 4.06 (q, $J=7.1$ Hz, 2H), 2.06-1.95 (m, 2H), 1.87-1.78 (m, 2H), 1.63 (d, $J=4.1 \mathrm{~Hz}, 3 \mathrm{H}), 1.36-1.26(\mathrm{~m}, 2 \mathrm{H})$, 1.25-1.04 (m, 8H), $1.00(\mathrm{t}, J=7.1 \mathrm{~Hz}, 3 \mathrm{H}) ; \quad{ }^{13} \mathrm{C} \mathrm{NMR}\left(100 \mathrm{MHz}, \mathrm{C}_{6} \mathrm{D}_{6}\right) \delta 166.2,149.1,131.9$, 124.9, 121.9, 60.0, 33.1, 32.3, 30.0, 29.6, 29.4, 28.3, 18.1, 14.4; HRMS (ESI) Calcd for $\mathrm{C}_{15} \mathrm{H}_{27} \mathrm{O}_{2}[\mathrm{M}+\mathrm{H}]^{+}$: 239.2006; Found: 239.2001.

\section{Table 2, entry 12}

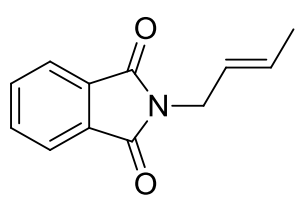

21

White solid; $\quad 0.0971 \mathrm{~g}(97 \%) ; \quad \mathrm{mp} .61 .1-63.6{ }^{\circ} \mathrm{C}$; IR (film) $1712,1674 \mathrm{~cm}^{-1} ; \quad{ }^{1} \mathrm{H}$ NMR $\left(400 \mathrm{MHz}, \mathrm{C}_{6} \mathrm{D}_{6}\right) \delta 7.43(\mathrm{dd}, J=5.4,3.0 \mathrm{~Hz}, 2 \mathrm{H}), 6.85(\mathrm{dd}, J=5.4,3.0 \mathrm{~Hz}, 2 \mathrm{H}), 5.66-5.50$ (m, $1 \mathrm{H}), 5.49-5.40(\mathrm{~m}, 1 \mathrm{H}), 4.08-4.03(\mathrm{~m}, 2 \mathrm{H}), 1.41-1.34(\mathrm{~m}, 3 \mathrm{H}) ;{ }^{13} \mathrm{C} \mathrm{NMR}\left(100 \mathrm{MHz}, \mathrm{C}_{6} \mathrm{D}_{6}\right) \delta$ 167.6, 133.5, 132.7, 129.6, 125.2, 123.0, 39.6, 17.6; HRMS (ESI) Calcd for $\mathrm{C}_{12} \mathrm{H}_{12} \mathrm{NO}_{2}$ $[\mathrm{M}+\mathrm{H}]^{+}: \quad 202.0863 ; \quad$ Found: 202.0864 .

\section{Table 2, entry 13}<smiles>C/C=C/c1ccccc1</smiles>

Colorless oil; $\quad 0.0502 \mathrm{~g}(85 \%) ; \quad$ IR (film) $1660 \mathrm{~cm}^{-1} ;{ }^{1} \mathrm{H}$ NMR (400 MHz, $\left.\mathrm{C}_{6} \mathrm{D}_{6}\right) \delta 7.24$ (d, $J=7.4 \mathrm{~Hz}, 2 \mathrm{H}), 7.14(\mathrm{t}, J=7.7 \mathrm{~Hz}, 2 \mathrm{H}), 7.04(\mathrm{t}, J=7.2 \mathrm{~Hz}, 1 \mathrm{H}), 6.30(\mathrm{~d}, J=15.8 \mathrm{~Hz}, 1 \mathrm{H})$, $6.02(\mathrm{dq}, J=15.6,6.6 \mathrm{~Hz}, 1 \mathrm{H}), 1.64(\mathrm{dd}, J=6.6,1.4 \mathrm{~Hz}, 3 \mathrm{H}) ;{ }^{13} \mathrm{C} \mathrm{NMR}\left(100 \mathrm{MHz}, \mathrm{C}_{6} \mathrm{D}_{6}\right) \delta$ 138.4, 131.7, 128.8, 127.1, 126.3, 125.5, 18.5; HRMS (ESI) Calcd for $\mathrm{C}_{9} \mathrm{H}_{14} \mathrm{~N}\left[\mathrm{M}+\mathrm{NH}_{4}\right]^{+}$: 136.1121; Found: 136.1125.

Wang, Y.; Qin, C.; Jia, X.; Leng, X.; Huang, Z. Angew. Chem. Int. Ed. 2017, 56, 1614. 


\section{Table 2, entry 14}

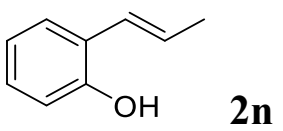

Light yellow oil; $0.0604 \mathrm{~g}(90 \%) ; \quad$ IR (film) 3415, $1658 \mathrm{~cm}^{-1} ;{ }^{1} \mathrm{H}$ NMR (400 MHz, $\mathrm{C}_{6} \mathrm{D}_{6}$ ) $\delta 7.30(\mathrm{dd}, J=7.6,1.7 \mathrm{~Hz}, 1 \mathrm{H}), 6.93(\mathrm{td}, J=7.6,1.8 \mathrm{~Hz}, 1 \mathrm{H}), 6.79(\mathrm{td}, J=7.5,1.2 \mathrm{~Hz}, 1 \mathrm{H})$, $6.70(\mathrm{dq}, J=15.9,2.0 \mathrm{~Hz}, 1 \mathrm{H}), 6.37$ (d, $J=8.0 \mathrm{~Hz}, 1 \mathrm{H}), 6.07(\mathrm{dq}, J=15.9,6.6 \mathrm{~Hz}, 1 \mathrm{H}), 4.43$ (s, 1H), $1.65(\mathrm{dd}, J=6.6,1.8 \mathrm{~Hz}, 3 \mathrm{H}) ;{ }^{13} \mathrm{C} \mathrm{NMR}\left(100 \mathrm{MHz}, \mathrm{C}_{6} \mathrm{D}_{6}\right) \delta 153.2,127.8,127.4$, 127.1, 126.1, 125.6, 121.0, 116.0, 18.9; $\quad$ HRMS (ESI) Calcd for $\mathrm{C}_{9} \mathrm{H}_{11} \mathrm{O}[\mathrm{M}+\mathrm{H}]^{+}: \quad$ 135.0804; Found: 135.0807.

Razzaq, T.; Glasnov, T.N.; Kappe, C.O. Chem. Eng. Technol. 2009, 32, 1702.

\section{Table 2, entry 15}<smiles>C/C=C/c1ccc(OC(C)=O)c(OC)c1</smiles>

White solid; $0.0998 \mathrm{~g}$ (97\%); mp. 75.3-77.3 ${ }^{\circ} \mathrm{C}$; $\quad$ IR (film) 1765, $1658 \mathrm{~cm}^{-1} ;{ }^{1} \mathrm{H}$ NMR $\left(400 \mathrm{MHz}, \mathrm{C}_{6} \mathrm{D}_{6}\right) \delta 6.96(\mathrm{~d}, J=8.1 \mathrm{~Hz}, 1 \mathrm{H}), 6.79(\mathrm{dd}, J=8.1,1.6 \mathrm{~Hz}, 1 \mathrm{H}), 6.72(\mathrm{~d}, J=1.6 \mathrm{~Hz}$, $1 \mathrm{H}), 6.25$ (dq, $J=15.8,1.3 \mathrm{~Hz}, 1 \mathrm{H}), 5.97$ (dq, $J=15.7,6.6 \mathrm{~Hz}, 1 \mathrm{H}), 3.30$ (s, 3H), 1.91 (s, 3H), $1.68(\mathrm{dd}, J=6.6,1.4 \mathrm{~Hz}, 3 \mathrm{H}) ; \quad{ }^{13} \mathrm{C} \mathrm{NMR}\left(100 \mathrm{MHz}, \mathrm{C}_{6} \mathrm{D}_{6}\right) \delta 168.3,151.8,139.6,137.1,131.1$, 125.6, 123.2, 118.6, 110.1, 55.3, 20.3, 18.4; HRMS (ESI) Calcd for $\mathrm{C}_{12} \mathrm{H}_{15} \mathrm{O}_{3}[\mathrm{M}+\mathrm{H}]^{+}$: 207.1016; Found: 207.1013.

Chakraborti, A.K.; Chankeshwara, S.V. J. Org. Chem. 2009, 74, 1367.

\section{Table 2, entry 16}

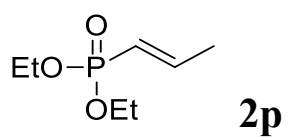

Light yellow oil; $0.0803 \mathrm{~g}(90 \%) ; \quad$ IR (film) $1637 \mathrm{~cm}^{-1} ; \quad{ }^{1} \mathrm{H}$ NMR (400 MHz, $\left.\mathrm{C}_{6} \mathrm{D}_{6}\right) 6.79$ (ddq, $J=21.5,17.0,6.6 \mathrm{~Hz}, 1 \mathrm{H}), 5.63-5.51(\mathrm{~m}, 1 \mathrm{H}), 4.02-3.85(\mathrm{~m}, 4 \mathrm{H}), 1.36(\mathrm{dt}, J=6.6,2.0$ $\mathrm{Hz}, 3 \mathrm{H}), 1.07$ (t, $J=7.0 \mathrm{~Hz}, 6 \mathrm{H}) ; \quad{ }^{13} \mathrm{C} \mathrm{NMR}\left(100 \mathrm{MHz}, \mathrm{C}_{6} \mathrm{D}_{6}\right) \delta 148.4$ (d, J=5.3 Hz), 120.1 $(\mathrm{d}, J=187.1 \mathrm{~Hz}), 61.3(\mathrm{~d}, J=5.3 \mathrm{~Hz}), 19.7(\mathrm{~d}, J=23.9 \mathrm{~Hz}), 16.5(\mathrm{~d}, J=6.1 \mathrm{~Hz}) ; \quad$ HRMS (ESI) Calcd for $\mathrm{C}_{7} \mathrm{H}_{16} \mathrm{O}_{3} \mathrm{P}[\mathrm{M}+\mathrm{H}]^{+}: \quad$ 179.0832; Found: 179.0830 . 
Teulade, M.-P.; Savignac, P.; Aboujaoude, E.E.; Liétge, S.; Collignon, N. J. Organomet. Chem. 1986, 304, 283.

\section{Table 2, entry 17}<smiles>[14CH3]/C=C/C(O)c1ccccc1</smiles>

Light yellow oil; $\quad 0.0601 \mathrm{~g}(81 \%) ; \quad$ IR (film) 3362, $1672 \mathrm{~cm}^{-1} ; \quad{ }^{1} \mathrm{H}$ NMR (400 MHz, $\left.\mathrm{C}_{6} \mathrm{D}_{6}\right)$ $\delta 7.31(\mathrm{~d}, J=7.5 \mathrm{~Hz}, 2 \mathrm{H}), 7.14(\mathrm{t}, J=7.4 \mathrm{~Hz}, 2 \mathrm{H}), 7.04(\mathrm{t}, J=7.3 \mathrm{~Hz}, 1 \mathrm{H}), 5.58-5.42(\mathrm{~m}, 2 \mathrm{H})$, $4.93(\mathrm{~d}, J=5.9 \mathrm{~Hz}, 1 \mathrm{H}), 2.55(\mathrm{br} \mathrm{s}, 1 \mathrm{H}), 1.44(\mathrm{~d}, J=5.6 \mathrm{~Hz}, 3 \mathrm{H}) ;{ }^{13} \mathrm{C} \mathrm{NMR}\left(100 \mathrm{MHz}, \mathrm{C}_{6} \mathrm{D}_{6}\right)$ $\delta 144.3,134.8,128.5,127.4,126.6,126.3,75.1,17.7$; HRMS (ESI) Calcd for $\mathrm{C}_{10} \mathrm{H}_{16} \mathrm{NO}$ $\left[\mathrm{M}+\mathrm{NH}_{4}\right]^{+}: \quad 166.1226$; Found: 166.1223 .

Akai, S.; Hanada, R.; Fujiwara, N.; Kita, Y.; Egi, M. Org. Lett. 2010, 12, 4900.

\section{Table 2, entry 18}<smiles>CC=CC1CCCCC1=O</smiles>

Colorless oil; $\quad 0.0630 \mathrm{~g}$ (91\%); IR (film) $1711 \mathrm{~cm}^{-1} ; \quad{ }^{1} \mathrm{H}$ NMR (400 MHz, $\left.\mathrm{C}_{6} \mathrm{D}_{6}\right) \delta 5.79$ (ddq, $J=15.4,7.1,1.7 \mathrm{~Hz}, 1 \mathrm{H}), 5.30(\mathrm{dqd}, J=15.5,6.5,1.2 \mathrm{~Hz}, 1 \mathrm{H}), 2.70-2.62(\mathrm{~m}, 1 \mathrm{H}), 2.21$ $(\mathrm{dtd}, J=13.6,4.7,1.4 \mathrm{~Hz}, 1 \mathrm{H}), 1.86(\mathrm{dddd}, J=13.6,11.6,5.7,1.2 \mathrm{~Hz}, 1 \mathrm{H}), 1.75-1.67(\mathrm{~m}, 1 \mathrm{H})$, $1.60(\mathrm{ddd}, J=6.4,1.7,0.9 \mathrm{~Hz}, 3 \mathrm{H}), 1.52-1.43$ (m, $1 \mathrm{H}), 1.41-1.21(\mathrm{~m}, 3 \mathrm{H}), 1.20-1.09(\mathrm{~m}, 1 \mathrm{H})$; ${ }^{13} \mathrm{C}$ NMR $\left(100 \mathrm{MHz}, \mathrm{C}_{6} \mathrm{D}_{6}\right) \delta 208.8,129.9,126.3,53.7,41.6,34.5,27.6,24.4,18.3 ; \quad$ HRMS (ESI) Calcd for $\mathrm{C}_{9} \mathrm{H}_{15} \mathrm{O}[\mathrm{M}+\mathrm{H}]^{+}:$139.1117; Found: 139.1113.

1) Larsen, C.R.; Grotjahn, D.B. J. Am. Chem. Soc. 2012, 134, 10357.

2) Erdogan, G.; Grotjahn, D.B. Org. Lett. 2014, 16, 2818.

\section{Table 2, entry 19}<smiles>CC1=CCC(c2ccccc2)CC1</smiles>

Light yellow oil; $\quad 0.0822 \mathrm{~g}(95 \%) ; \quad$ IR (film) $1678 \mathrm{~cm}^{-1} ; \quad{ }^{1} \mathrm{H}$ NMR $\left(400 \mathrm{MHz}, \mathrm{C}_{6} \mathrm{D}_{6}\right) \delta 7.23-$ 
$7.17(\mathrm{~m}, 2 \mathrm{H}), 7.15-7.07(\mathrm{~m}, 3 \mathrm{H})$, 5.46-5.40 (m, 1H), 2.69-2.59 (m, 1H), 2.26-2.17 (m, 1H), 2.17-2.06 (m, 1H), 2.03-1.91 (m, 1H), 1.88-1.77 (m, 2H), 1.73-1.65 (m, $1 \mathrm{H}), 1.63(\mathrm{~s}, 3 \mathrm{H}) ; \quad{ }^{13} \mathrm{C}$ NMR (100 MHz, $\left.\mathrm{C}_{6} \mathrm{D}_{6}\right) \delta 147.5,133.7,128.7,127.2,126.3,121.4,40.4,34.0,30.9,30.5,23.8$; HRMS (ESI) Calcd for $\mathrm{C}_{13} \mathrm{H}_{16} \mathrm{Na}[\mathrm{M}+\mathrm{Na}]^{+}:$195.1144; Found: 195.1146 .

Liu, X.; Zhang, W.; Wang, Y.; Zhang, Z.-X.; Jiao, L.; Liu, Q. J. Am. Chem. Soc. 2018, 140, 6873.

\section{Table 2, entry 20}

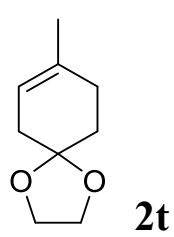

Light yellow oil; $\quad 0.0686 \mathrm{~g}(89 \%) ; \quad$ IR (film) $1660 \mathrm{~cm}^{-1} ; \quad{ }^{1} \mathrm{H}$ NMR (400 MHz, $\left.\mathrm{C}_{6} \mathrm{D}_{6}\right) \delta 5.29$ $5.23(\mathrm{~m}, 1 \mathrm{H}), 3.55(\mathrm{~s}, 4 \mathrm{H}), 2.32(\mathrm{~s}, 2 \mathrm{H}), 2.11(\mathrm{t}, J=6.9 \mathrm{~Hz}, 2 \mathrm{H}), 1.77(\mathrm{t}, J=6.6 \mathrm{~Hz}, 2 \mathrm{H}), 1.59$ $(\mathrm{s}, 3 \mathrm{H}) ; \quad{ }^{13} \mathrm{C}$ NMR $\left(100 \mathrm{MHz}, \mathrm{C}_{6} \mathrm{D}_{6}\right) \delta 133.5,119.3,108.2,64.3,36.2,31.8,29.6,23.3$; HRMS (ESI) Calcd for $\mathrm{C}_{9} \mathrm{H}_{18} \mathrm{NO}_{2}\left[\mathrm{M}+\mathrm{NH}_{4}\right]^{+}: \quad$ 172.1332; Found: 172.1334 .

\section{Table 2, entry 21}

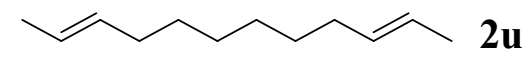

Colorless oil; $0.0782 \mathrm{~g}(94 \%)$; IR (film) $1644 \mathrm{~cm}^{-1} ; \quad{ }^{1} \mathrm{H}$ NMR (400 MHz, $\left.\mathrm{C}_{6} \mathrm{D}_{6}\right) \delta 5.52-$ $5.36(\mathrm{~m}, 4 \mathrm{H}), 2.06-1.95(\mathrm{~m}, 4 \mathrm{H}), 1.62(\mathrm{~d}, J=4.6 \mathrm{~Hz}, 6 \mathrm{H}), 1.40-1.20(\mathrm{~m}, 8 \mathrm{H}) ; \quad{ }^{13} \mathrm{C} \mathrm{NMR}(100$ $\left.\mathrm{MHz}, \mathrm{C}_{6} \mathrm{D}_{6}\right) \delta 132.0,124.9,33.1,30.0,29.5,18.1$; HRMS (ESI) Calcd for $\mathrm{C}_{13} \mathrm{H}_{27} \mathrm{O}$ $[\mathrm{M}+\mathrm{MeOH}+\mathrm{H}]^{+}: \quad$ 199.2056; Found: 199.2053 .

\section{Table 2, entry 22}

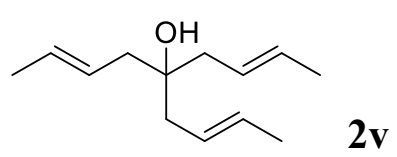

Light yellow oil; $0.0903 \mathrm{~g}(93 \%) ; \quad$ IR (film) 3470, $1666 \mathrm{~cm}^{-1} ; \quad{ }^{1} \mathrm{H}$ NMR (400 MHz, $\left.\mathrm{C}_{6} \mathrm{D}_{6}\right)$

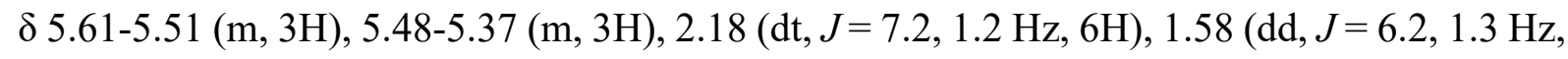
9H), $1.38(\mathrm{~s}, 1 \mathrm{H}) ; \quad{ }^{13} \mathrm{C} \mathrm{NMR}\left(100 \mathrm{MHz}, \mathrm{C}_{6} \mathrm{D}_{6}\right) \delta 128.6,127.2,73.5,43.0,18.3 ; \quad$ HRMS (ESI) Calcd for $\mathrm{C}_{13} \mathrm{H}_{22} \mathrm{KO}[\mathrm{M}+\mathrm{K}]^{+}$: 233.1302; Found: 233.1298 . 


\section{Table 2, entry 23}

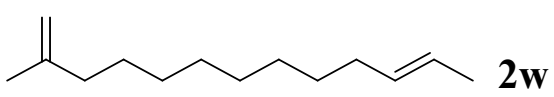

Colorless oil; $\quad 0.0921 \mathrm{~g}(95 \%)$; IR (film) $1652 \mathrm{~cm}^{-1} ; \quad{ }^{1} \mathrm{H}$ NMR (400 MHz, $\left.\mathrm{C}_{6} \mathrm{D}_{6}\right) \delta 5.53-$ $5.37(\mathrm{~m}, 2 \mathrm{H}), 4.81(\mathrm{~s}, 2 \mathrm{H}), 2.10-1.94(\mathrm{~m}, 4 \mathrm{H}), 1.66(\mathrm{~s}, 3 \mathrm{H}), 1.62$ (d, J=4.8 Hz, 3H), 1.46-1.22 $(\mathrm{m}, 12 \mathrm{H}) ; \quad{ }^{13} \mathrm{C} \mathrm{NMR}\left(100 \mathrm{MHz}, \mathrm{C}_{6} \mathrm{D}_{6}\right) \delta$ 146.0, 132.0, 124.9, 110.2, 38.2, 33.2, 30.1, 30.0, 29.8, 29.7, 28.1, 22.5, 18.2; HRMS (ESI) Calcd for $\mathrm{C}_{15} \mathrm{H}_{31} \mathrm{O}[\mathrm{M}+\mathrm{MeOH}+\mathrm{H}]^{+}: \quad$ 227.2369; Found: 227.2367

\section{Table 2, entry 24}

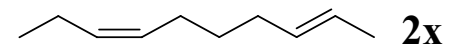

Colorless oil; $\quad 0.0632 \mathrm{~g}(91 \%) ; \quad$ IR (film) $1672 \mathrm{~cm}^{-1} ; \quad{ }^{1} \mathrm{H}$ NMR (400 MHz, $\left.\mathrm{C}_{6} \mathrm{D}_{6}\right) \delta 5.50-$ $5.34(\mathrm{~m}, 4 \mathrm{H}), 2.10-1.93(\mathrm{~m}, 6 \mathrm{H}), 1.63-1.58(\mathrm{~m}, 3 \mathrm{H}), 1.45-1.36(\mathrm{~m}, 2 \mathrm{H}), 0.92(\mathrm{t}, J=7.5 \mathrm{~Hz}, 3 \mathrm{H})$; ${ }^{13} \mathrm{C}$ NMR (100 MHz, $\left.\mathrm{C}_{6} \mathrm{D}_{6}\right) \delta 132.0,131.7,129.3,125.1,32.6,30.1,27.0,21.0,18.1,14.6$; HRMS (ESI) Calcd for $\mathrm{C}_{10} \mathrm{H}_{18} \mathrm{~K}[\mathrm{M}+\mathrm{K}]^{+}: \quad 177.1040 ; \quad$ Found: $\quad 177.1040$.

\section{Scheme 3, 2y}

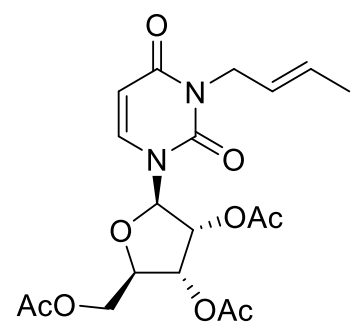

Colorless oil; $0.1918 \mathrm{~g}$ (90\%); IR (film) 1750, 1712, $1671 \mathrm{~cm}^{-1} ;{ }^{1} \mathrm{H}$ NMR (400 MHz, $\left.\mathrm{C}_{6} \mathrm{D}_{6}\right) \delta 7.12(\mathrm{~d}, J=8.2 \mathrm{~Hz}, 1 \mathrm{H}), 6.10(\mathrm{~d}, J=4.9 \mathrm{~Hz}, 1 \mathrm{H}), 5.79-5.68(\mathrm{~m}, 1 \mathrm{H}), 5.63(\mathrm{~d}, J=8.1$ $\mathrm{Hz}, 1 \mathrm{H}), 5.52(\mathrm{t}, J=5.9 \mathrm{~Hz}, 2 \mathrm{H}), 5.41(\mathrm{t}, J=5.6 \mathrm{~Hz}, 1 \mathrm{H}), 4.44(\mathrm{~d}, J=6.3 \mathrm{~Hz}, 2 \mathrm{H}), 4.21(\mathrm{dd}, J$ $=12.4,4.4 \mathrm{~Hz}, 1 \mathrm{H}), 4.17(\mathrm{dd}, J=12.4,3.3 \mathrm{~Hz}, 1 \mathrm{H}), 4.12-4.05(\mathrm{~m}, 1 \mathrm{H}), 1.71(\mathrm{~s}, 3 \mathrm{H}), 1.68(\mathrm{~s}$, $3 \mathrm{H}), 1.67(\mathrm{~s}, 3 \mathrm{H}), 1.42(\mathrm{dd}, J=6.5,1.6 \mathrm{~Hz}, 3 \mathrm{H}) ; \quad{ }^{13} \mathrm{C} \mathrm{NMR}\left(100 \mathrm{MHz}, \mathrm{C}_{6} \mathrm{D}_{6}\right) \delta 169.9,169.44$, $169.4,161.7,151.0,137.4,130.3,125.1,102.9,89.0,80.0,73.4,70.4,63.1,42.7,20.2,20.0$, 19.9, 17.7; HRMS (ESI) Calcd for $\mathrm{C}_{19} \mathrm{H}_{25} \mathrm{~N}_{2} \mathrm{O}_{9}[\mathrm{M}+\mathrm{H}]^{+}: \quad 425.1555 ; \quad$ Found: 425.1551 . 


\section{Scheme 3, 2z}

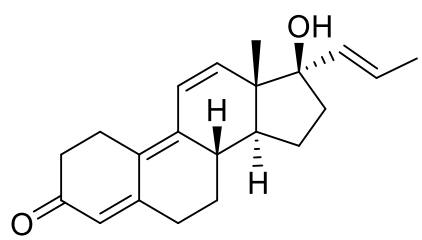

Light yellow oil; $\quad 0.1283 \mathrm{~g}(83 \%) ; \quad$ IR (film) 3425, 1641, $1570 \mathrm{~cm}^{-1} ; \quad{ }^{1} \mathrm{H}$ NMR (400 MHz, $\left.\mathrm{C}_{6} \mathrm{D}_{6}\right) \delta 6.22(\mathrm{~d}, J=10.0 \mathrm{~Hz}, 1 \mathrm{H}), 6.15(\mathrm{~d}, J=10.0 \mathrm{~Hz}, 1 \mathrm{H}), 5.89(\mathrm{~s}, 1 \mathrm{H}), 5.62-5.46(\mathrm{~m}, 2 \mathrm{H})$, 2.35-2.28 (m, 2H), 2.26-2.20 (m, 2H), 2.16-2.05 (m, 3H), 1.95-1.85 (m, 1H), 1.83-1.73 (m, 1H), $1.63-1.55(\mathrm{~m}, 1 \mathrm{H}), 1.53(\mathrm{~d}, J=5.9 \mathrm{~Hz}, 3 \mathrm{H}), 1.49-1.41(\mathrm{~m}, 1 \mathrm{H}), 1.41-1.33(\mathrm{~m}, 1 \mathrm{H}), 1.26-1.12$ (m, 2H), $1.02(\mathrm{~s}, 3 \mathrm{H}), 1.00-0.91(\mathrm{~m}, 1 \mathrm{H}) ;{ }^{13} \mathrm{C} \mathrm{NMR}\left(100 \mathrm{MHz}, \mathrm{C}_{6} \mathrm{D}_{6}\right) \delta$ 198.0, 155.6, 142.0, $141.6,137.0,127.2,124.2,124.0,123.9,81.6,49.4,48.1,38.5,36.9,36.5,31.5,27.3,24.5$, 23.1, 18.1, 16.8; HRMS (ESI) Calcd for $\mathrm{C}_{21} \mathrm{H}_{27} \mathrm{O}_{2}[\mathrm{M}+\mathrm{H}]^{+}: \quad 311.2006 ; \quad$ Found: 311.1998 .

Procedure for the gram scale of the isomerization of 1-dodecene (1a) (Scheme 4)

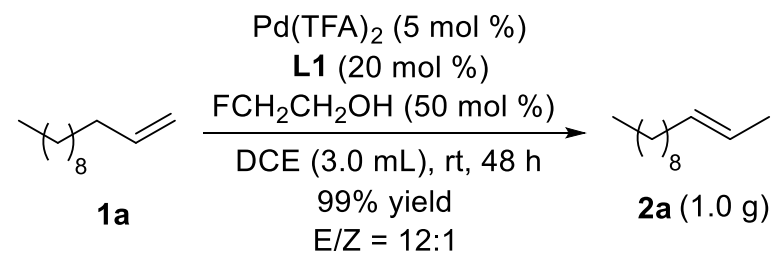

To a mixture of Pd(TFA $)_{2}(0.0997 \mathrm{~g}, 0.30 \mathrm{mmol})$ and ligand $\mathbf{L 1}(0.316 \mathrm{~g}, 1.20 \mathrm{mmol})$ in a vial (4.0 mL) were added 2-fluoroethanol (0.192 g, $3.0 \mathrm{mmol})$, 1-dodecene (1a) (1.01 g, $6.0 \mathrm{mmol})$, and dry DCE $(3.0 \mathrm{~mL})$ successively via syringe. The vial was purged with Ar to remove the air and tightly sealed with a septum cap. The reaction mixture was stirred at room temperature for $48 \mathrm{~h}$ and purified by flash chromatography (silica gel, eluent: petroleum ether) to give olefin 2a as a colorless oil (1.0 g, 99\% yield). 


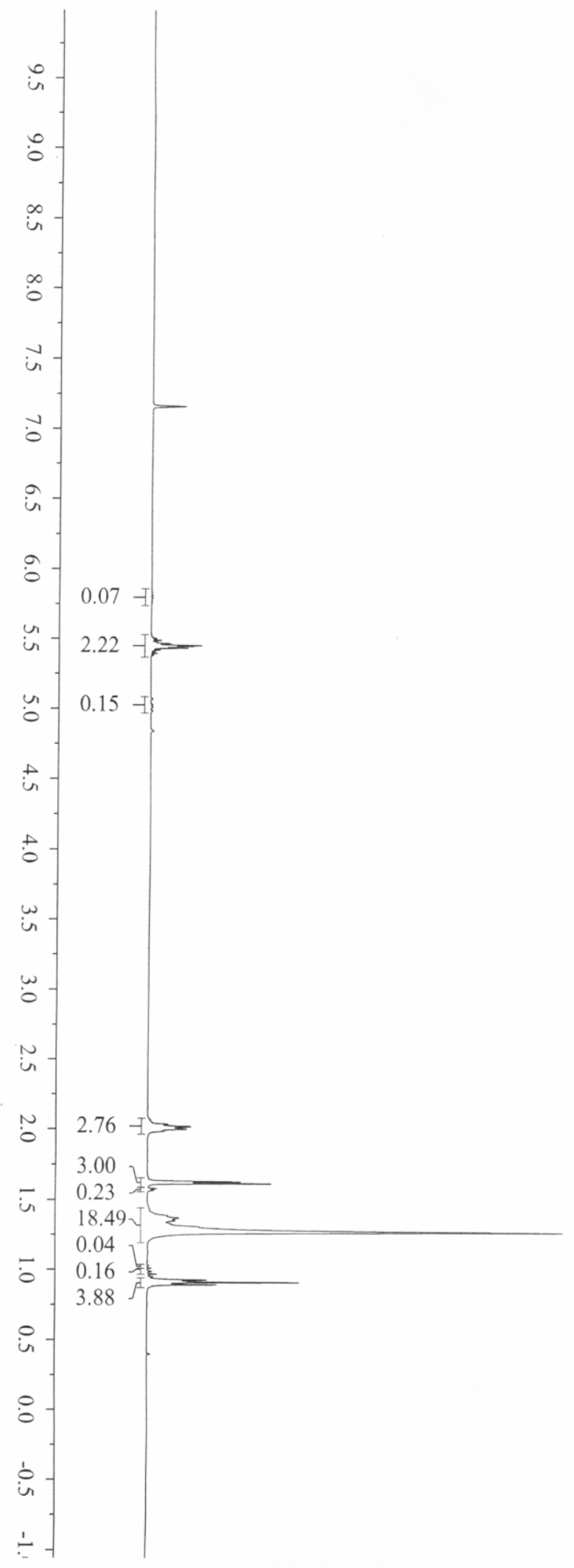




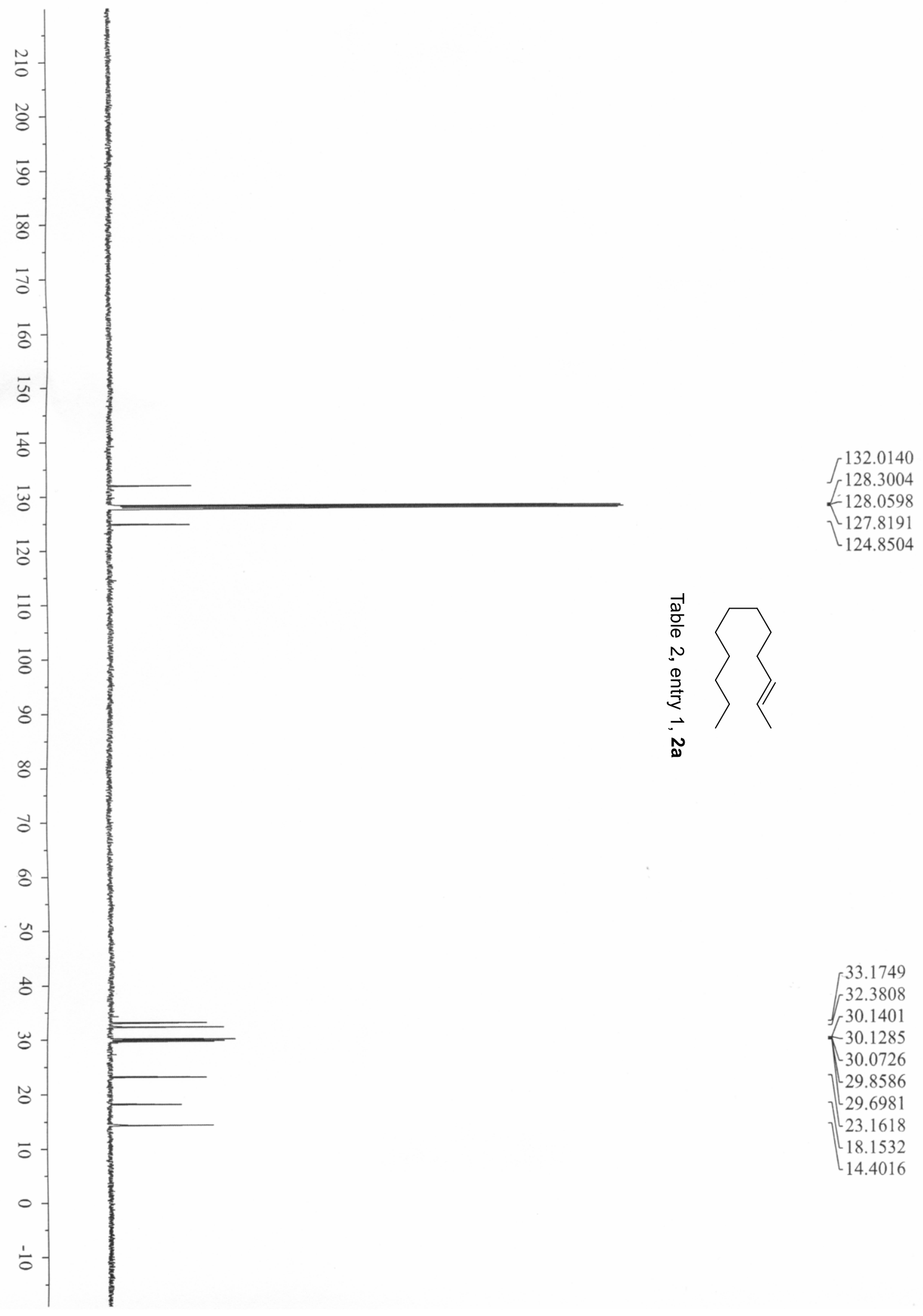




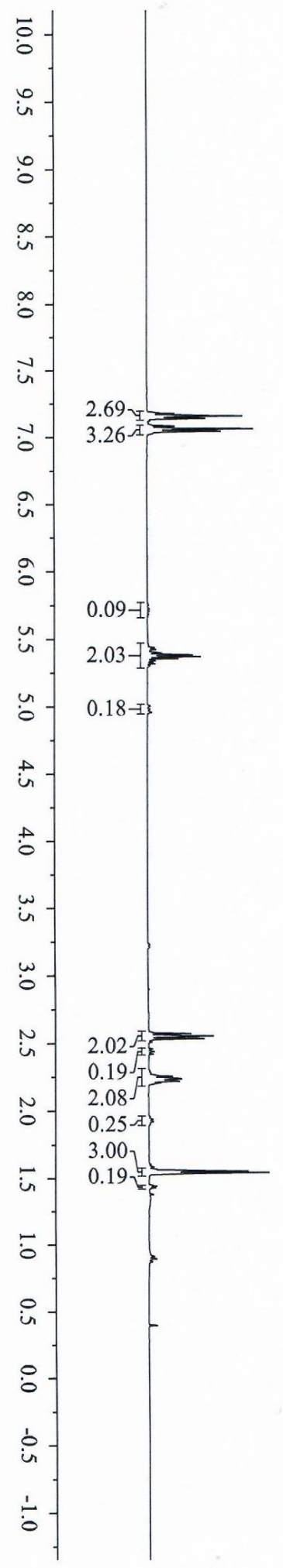

7.1818

$-7.1791$

$-7.1649$

$-7.1598$

$-7.1468$

$-7.1421$

$-7.0884$

$-7.0845$

7.0812

$-7.0655$

7.0612

$-7.0491$

7.0446

$-7.0312$

5.4382

5.4297

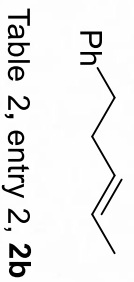

5.4252
5.4169

5.4122

$-5.4068$

$-5.4034$

$-5.4003$

$-5.3866$

$-5.3787$

$-5.3726$

$-5.3589$

$-5.3468$

$-5.3444$

$-5.3413$

$-5.3340$

$-5.3213$

$-5.3189$

5.3064

2.5731

$-2.5547$

$-2.5491$

$-2.5339$

$-2.2815$

$-2.2585$

$-2.2432$

$-2.2398$

$-2.2364$

$-2.2259$

$-2.2209$

$-2.2174$

$-2.2070$

$-2.2025$

$-2.1983$

$-1.5532$

$-1.5413$

$-1.4426$

1 12ก5 


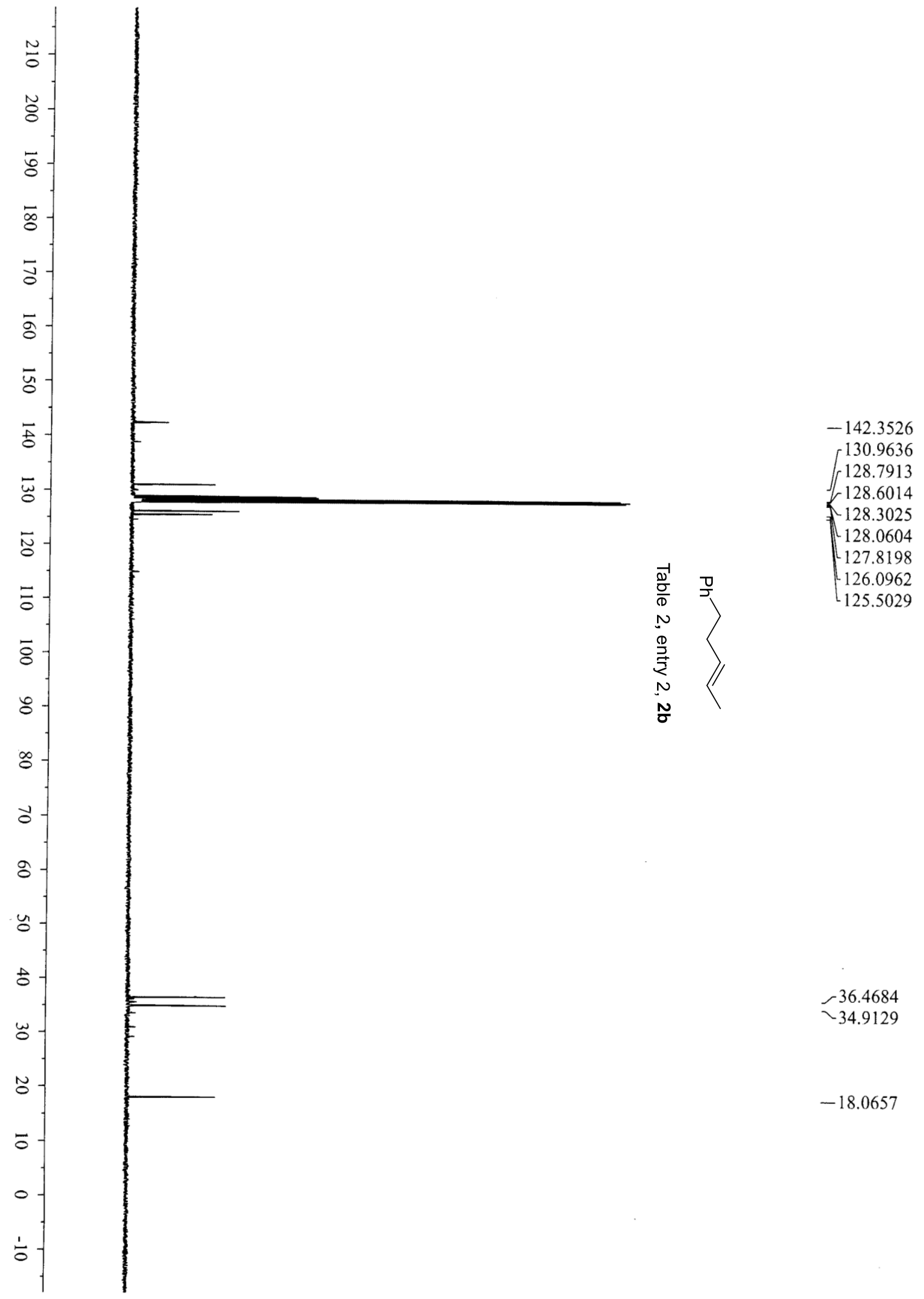



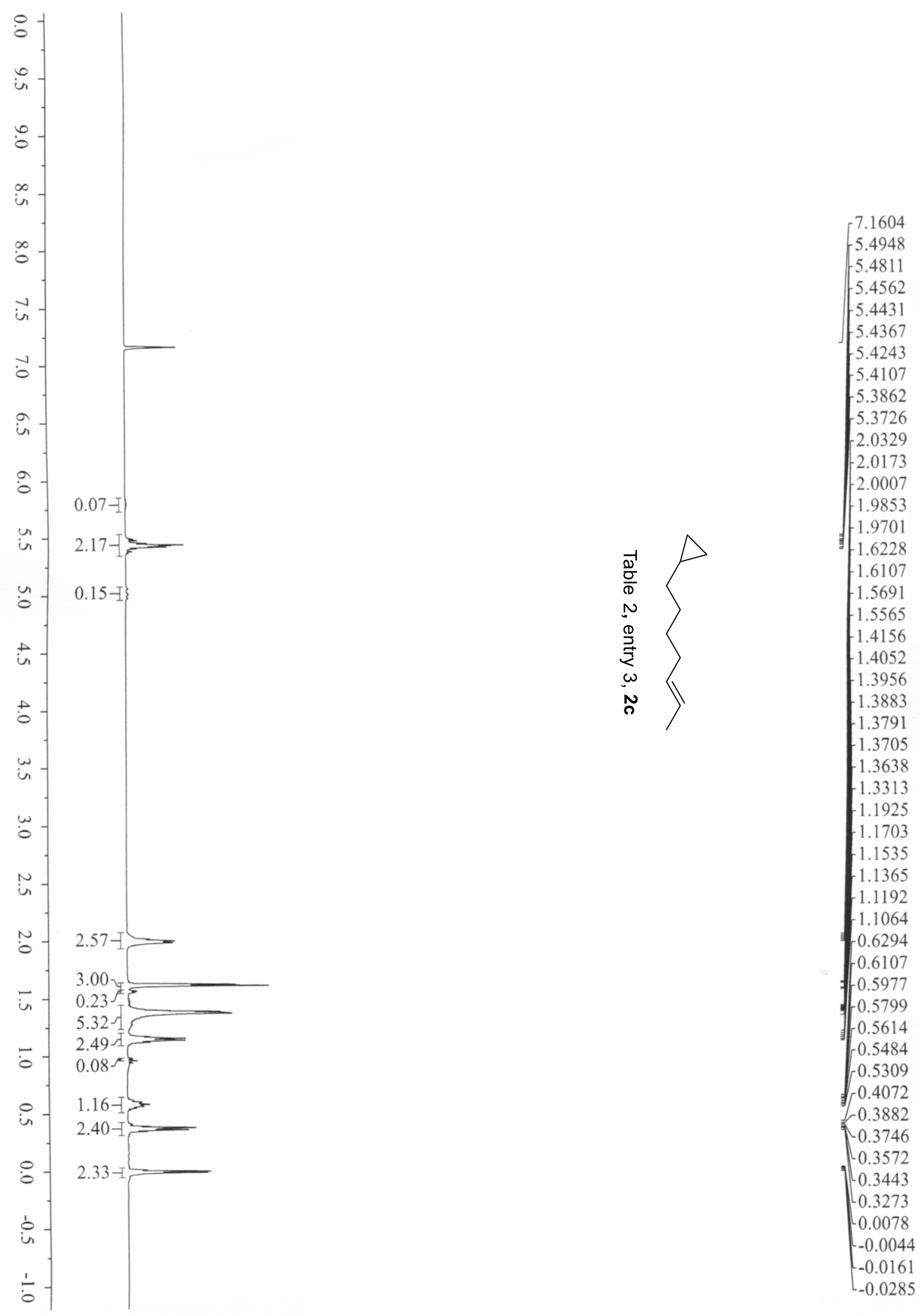


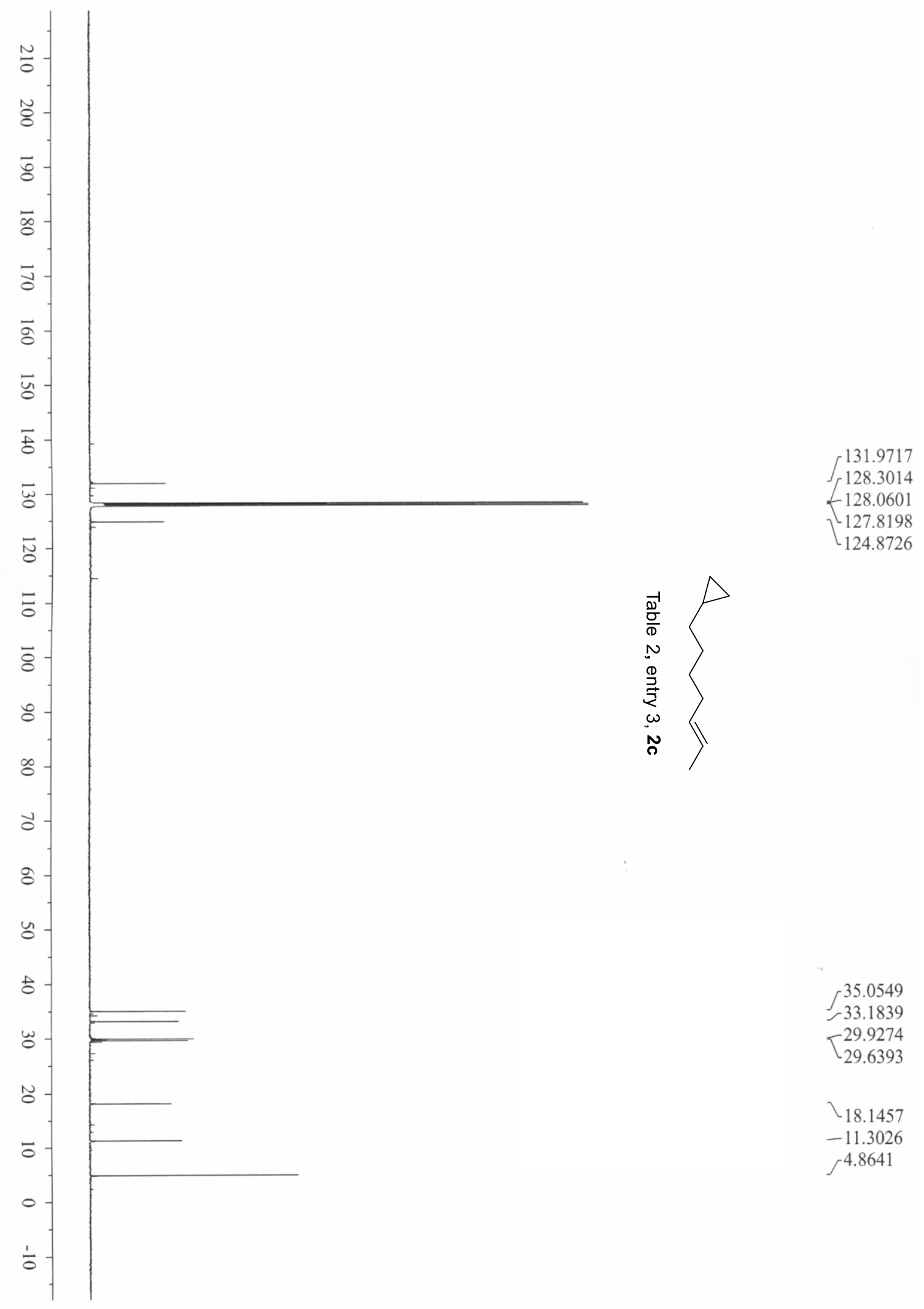



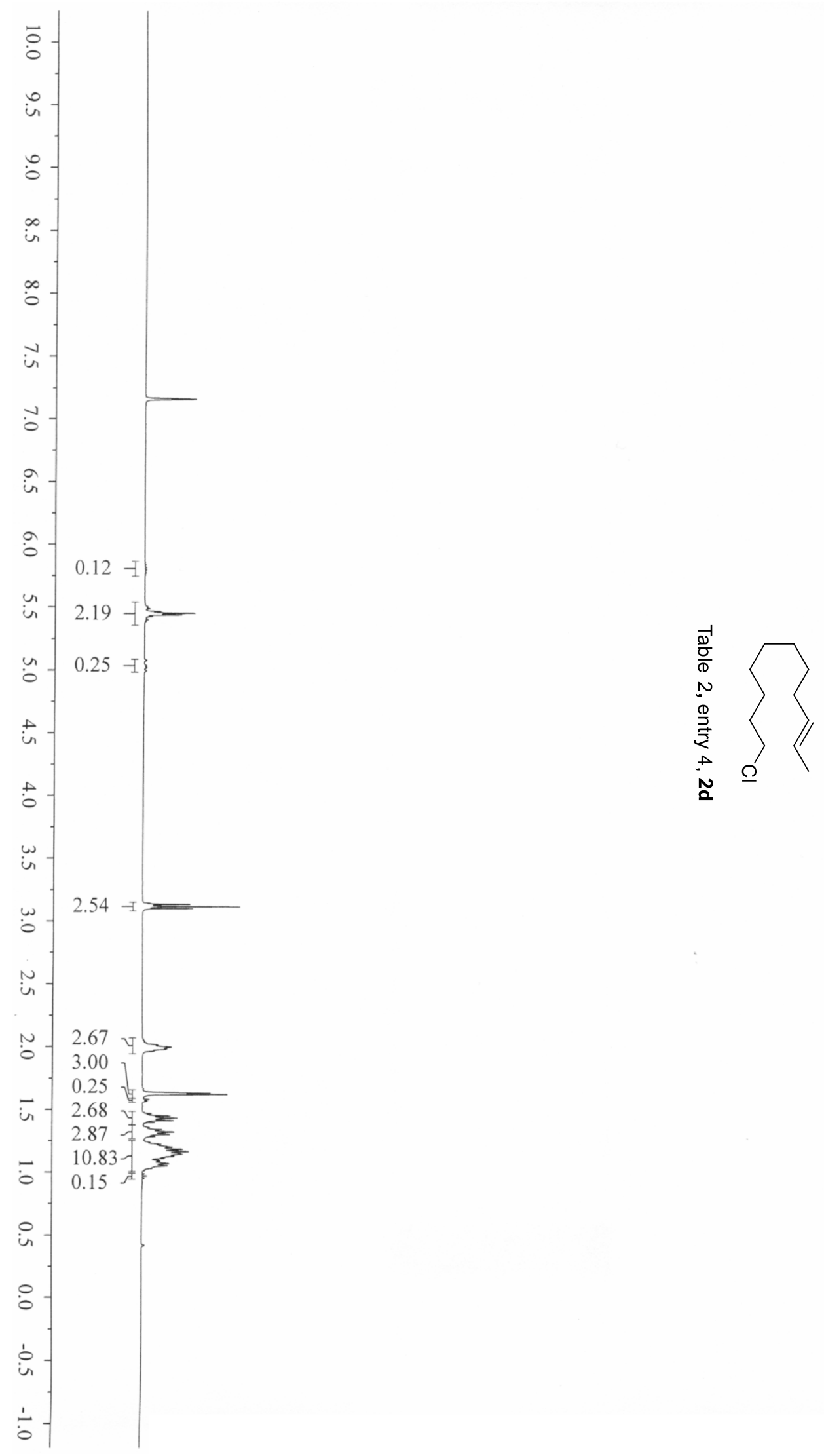

$\left[\begin{array}{l}5.5079 \\ 5.5023 \\ 5.4948 \\ 5.4888 \\ 5.4639 \\ 5.4508 \\ 5.4442 \\ 5.4383 \\ 5.4254 \\ 5.4130 \\ 5.4004 \\ 5.3873\end{array}\right.$

3.1340

$-3.1172$

3.1003

2.0136

1.9990

$-1.9855$

$-1.9702$

1.6355

1.6243

1.4687

1.4518

1.4344

1.4147

1.3978

$\left\{\begin{array}{l}1.3413 \\ 1.3228\end{array}\right.$

1.3032

1.2854

1.2385

$-1.2233$

$-1.2026$

$-1.2016$

$-1.1834$

$-1.1660$

$-1.1507$

$-1.1448$

$-1.0700$ 


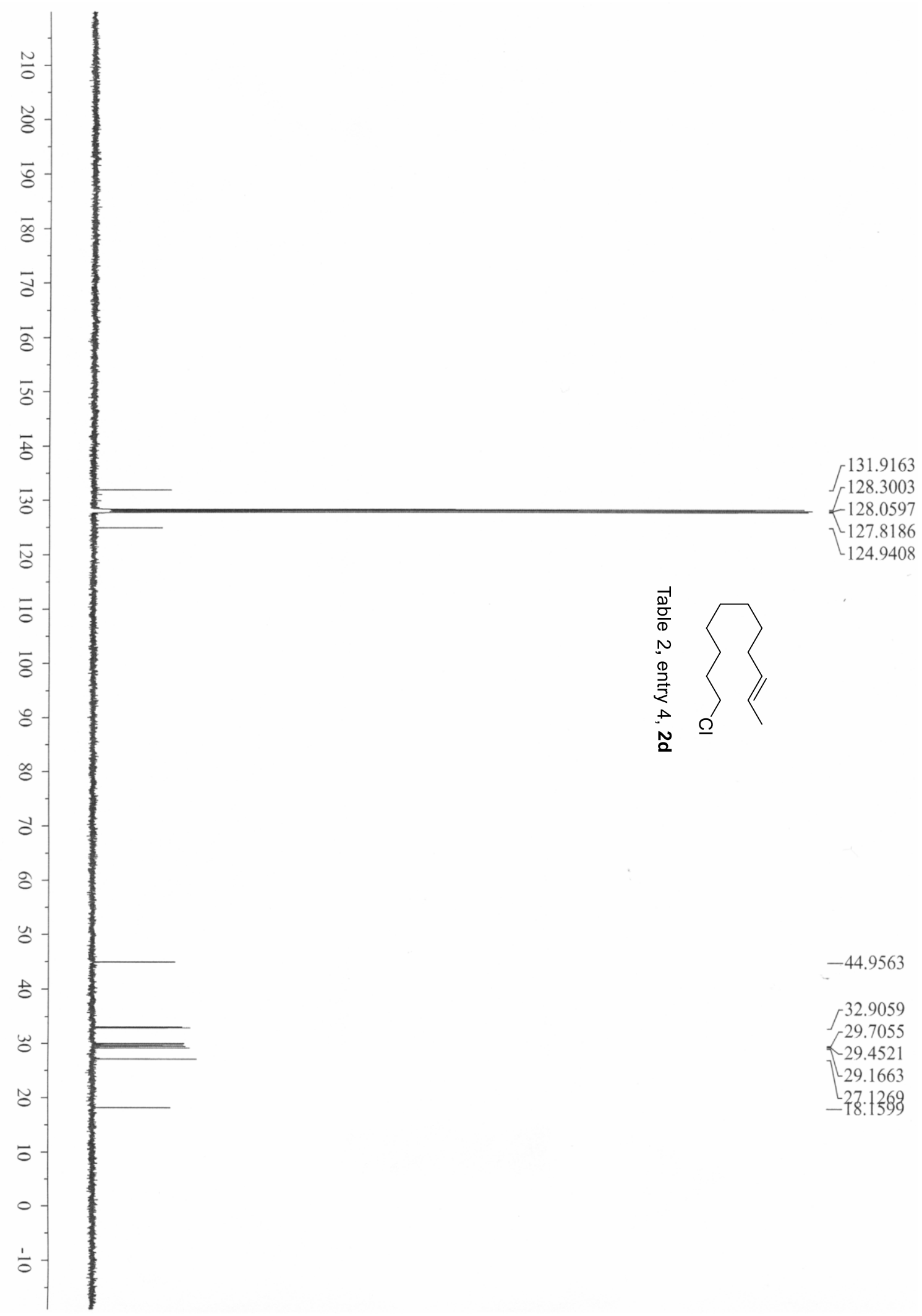




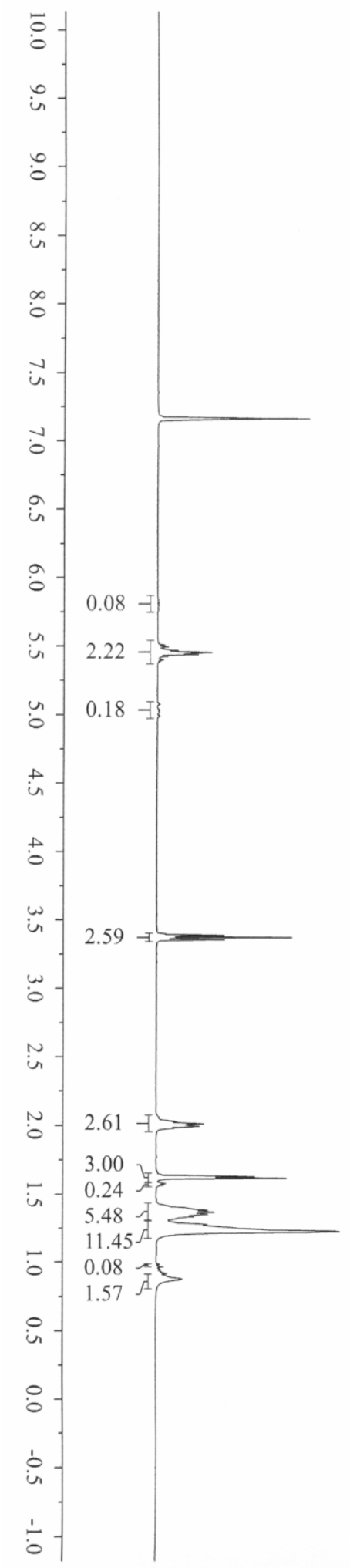

$-5.4236$

5.3990

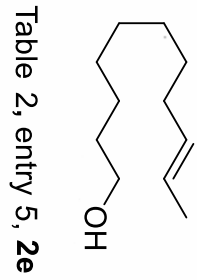

$\int_{-3.3710}^{3.3871}$

3.3547

2.0281

2.0124

1.9947

1.9810

1.6301

1.6184

1.5788

1.5663

$-1.3848$

1.3684

1.3528

1.3357

$-1.3192$

$-1.2774$

1.2278 


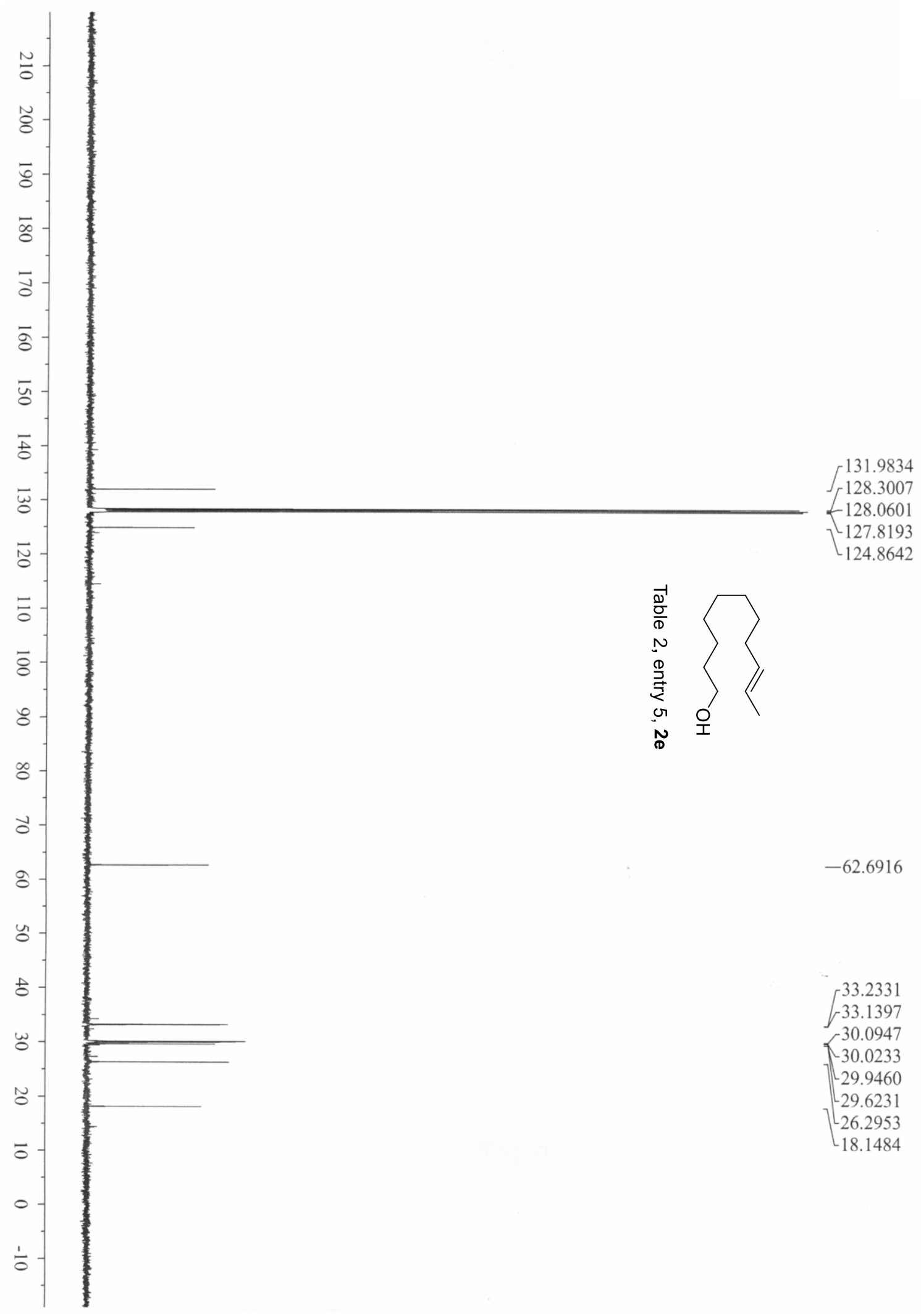



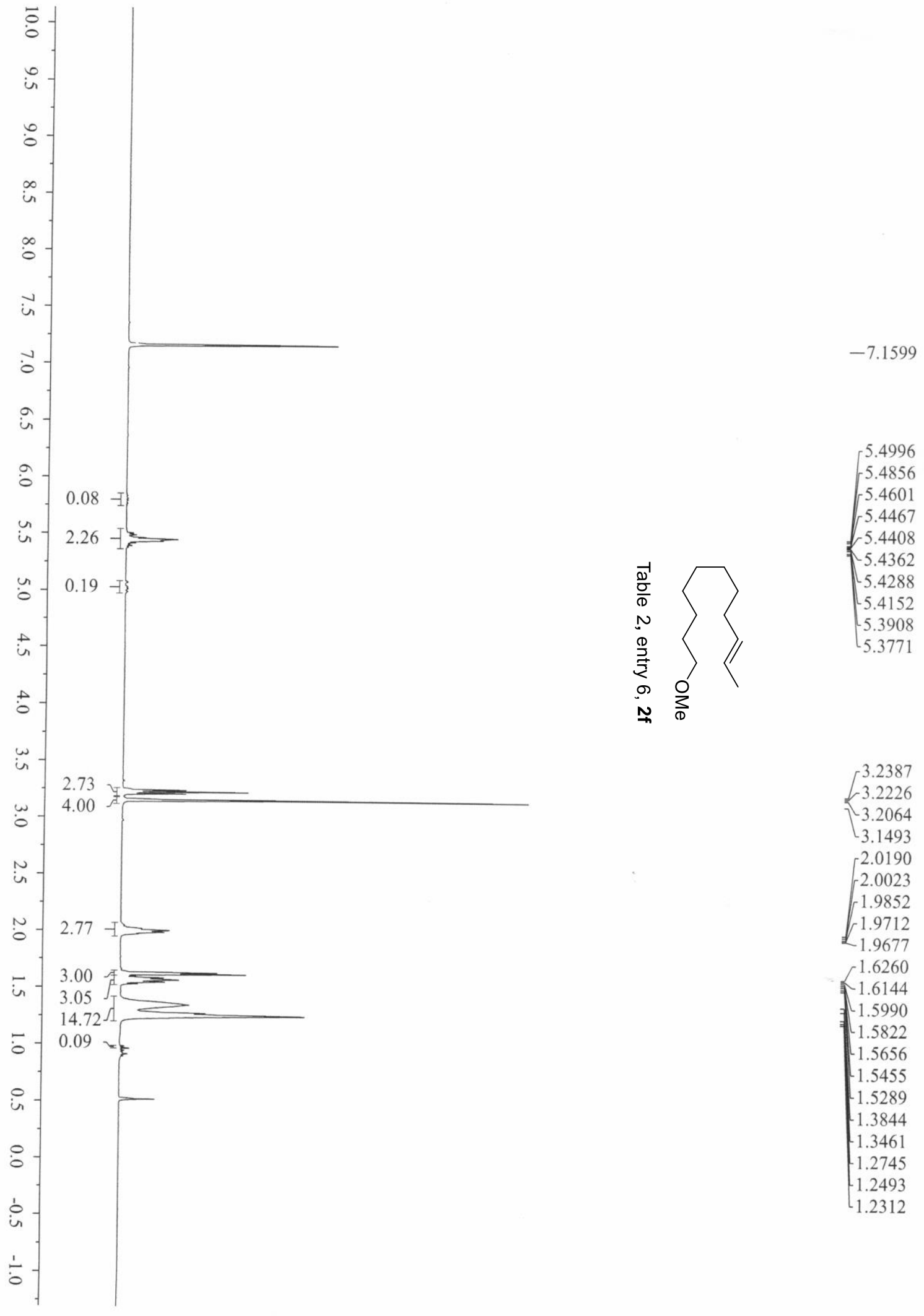

3.2387

3.2226

3.2064

3.1493

2.0190

2.0023

1.9852

1.9712
-1.9677

1.6260

1.6144

1.5990

1.5822

1.5656

1.5455

$-1.5289$

$-1.3844$

1.3461

$-1.2745$

$-1.2493$

1.2312 


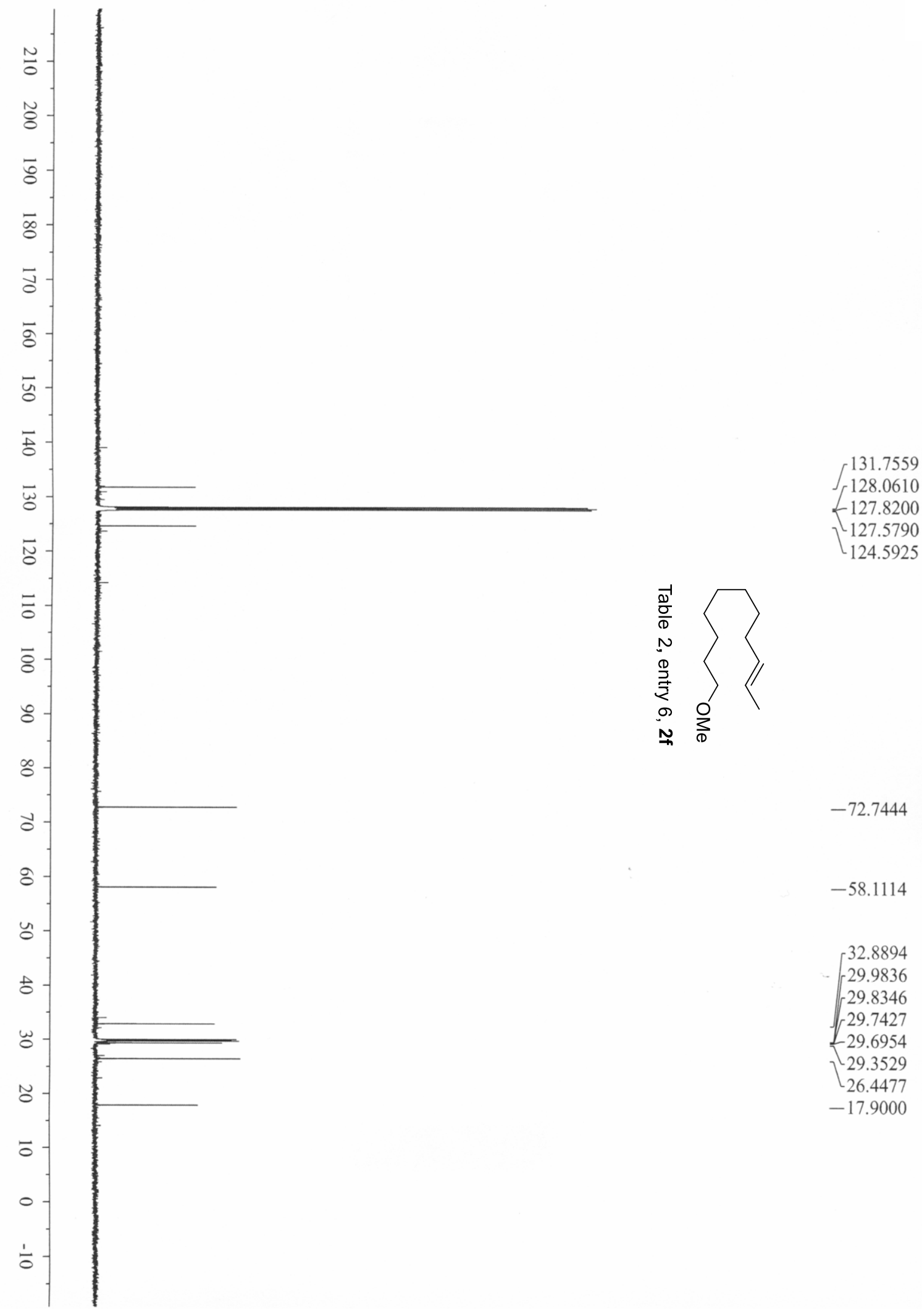




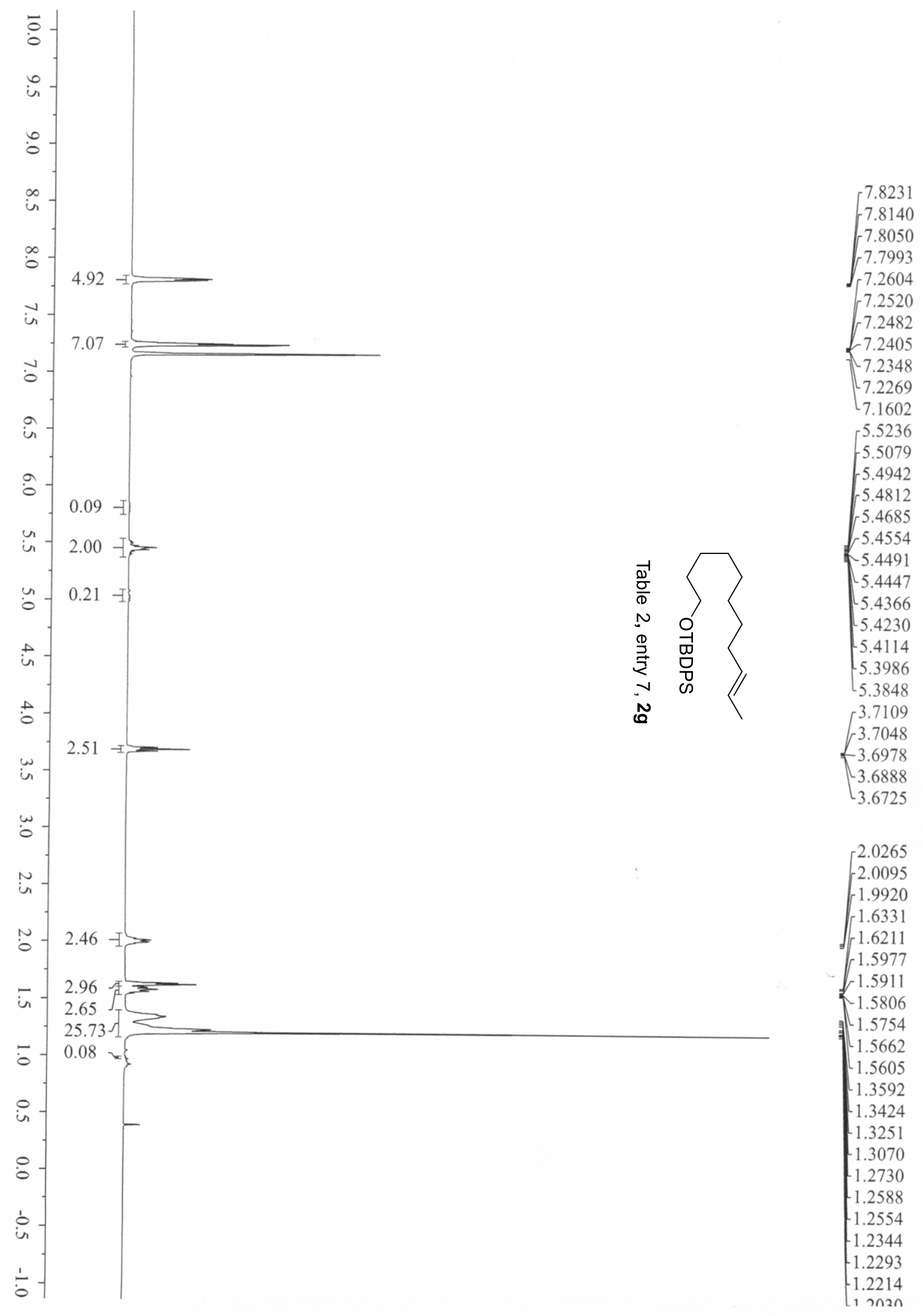




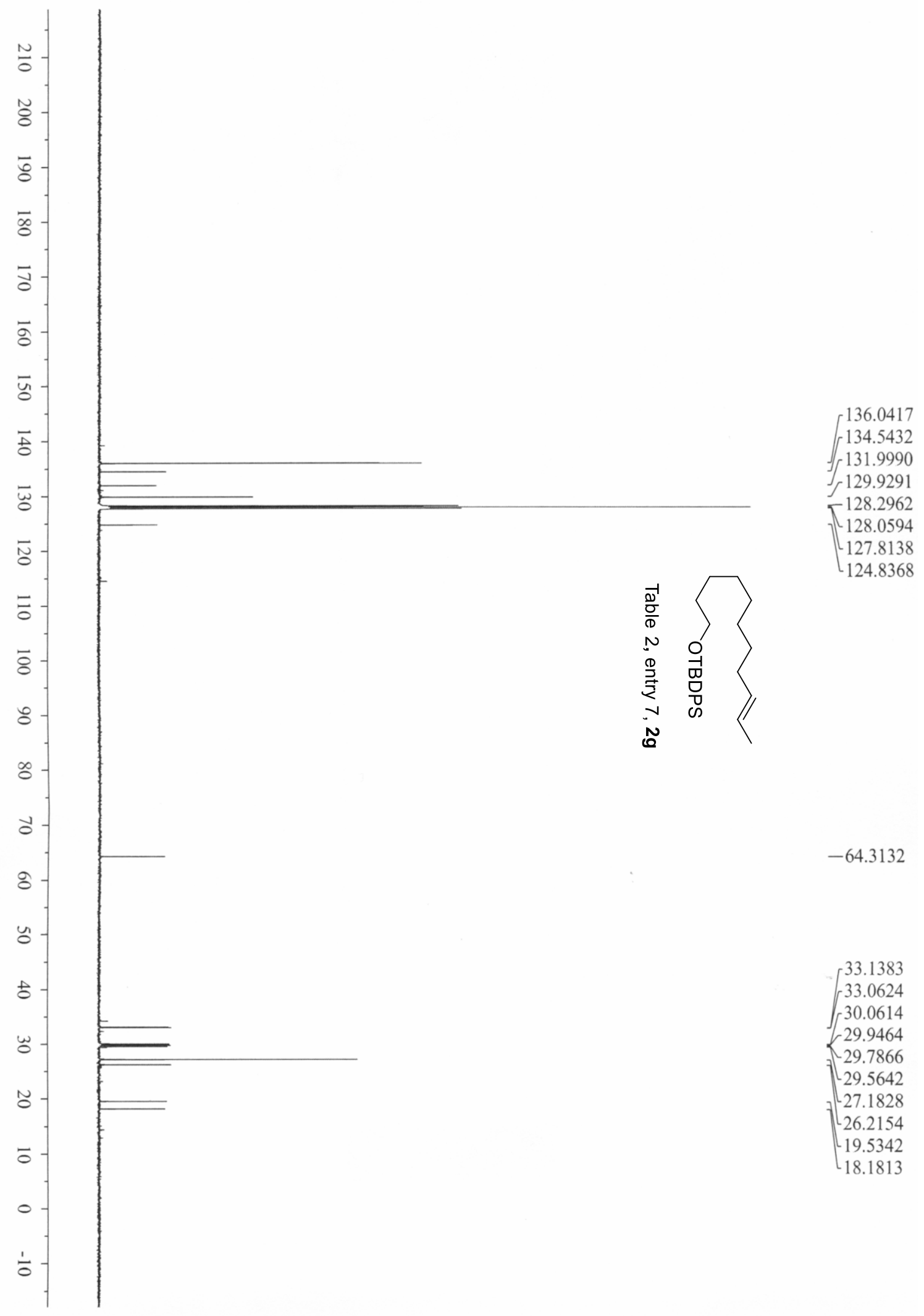




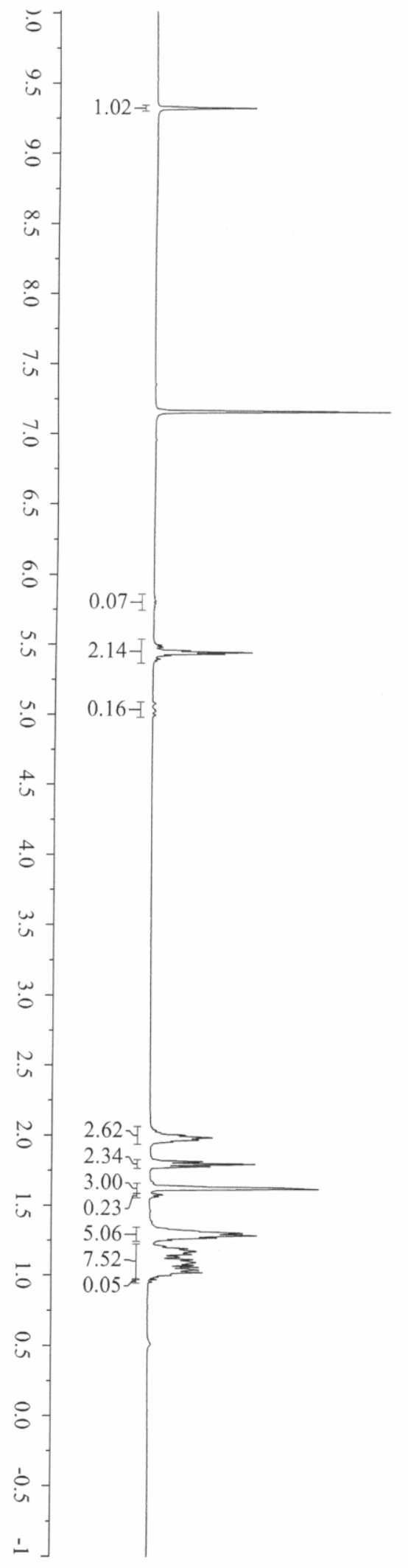




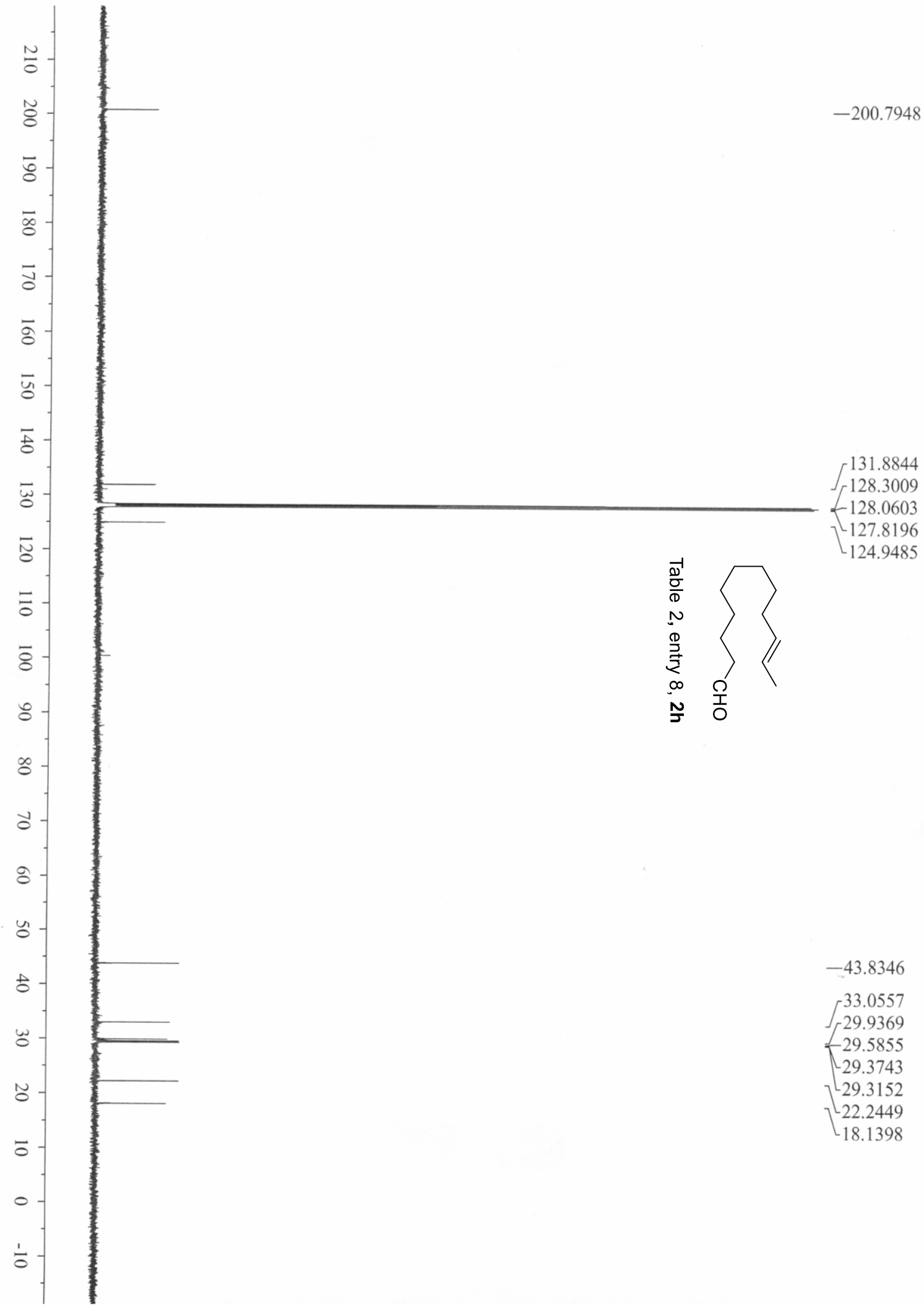




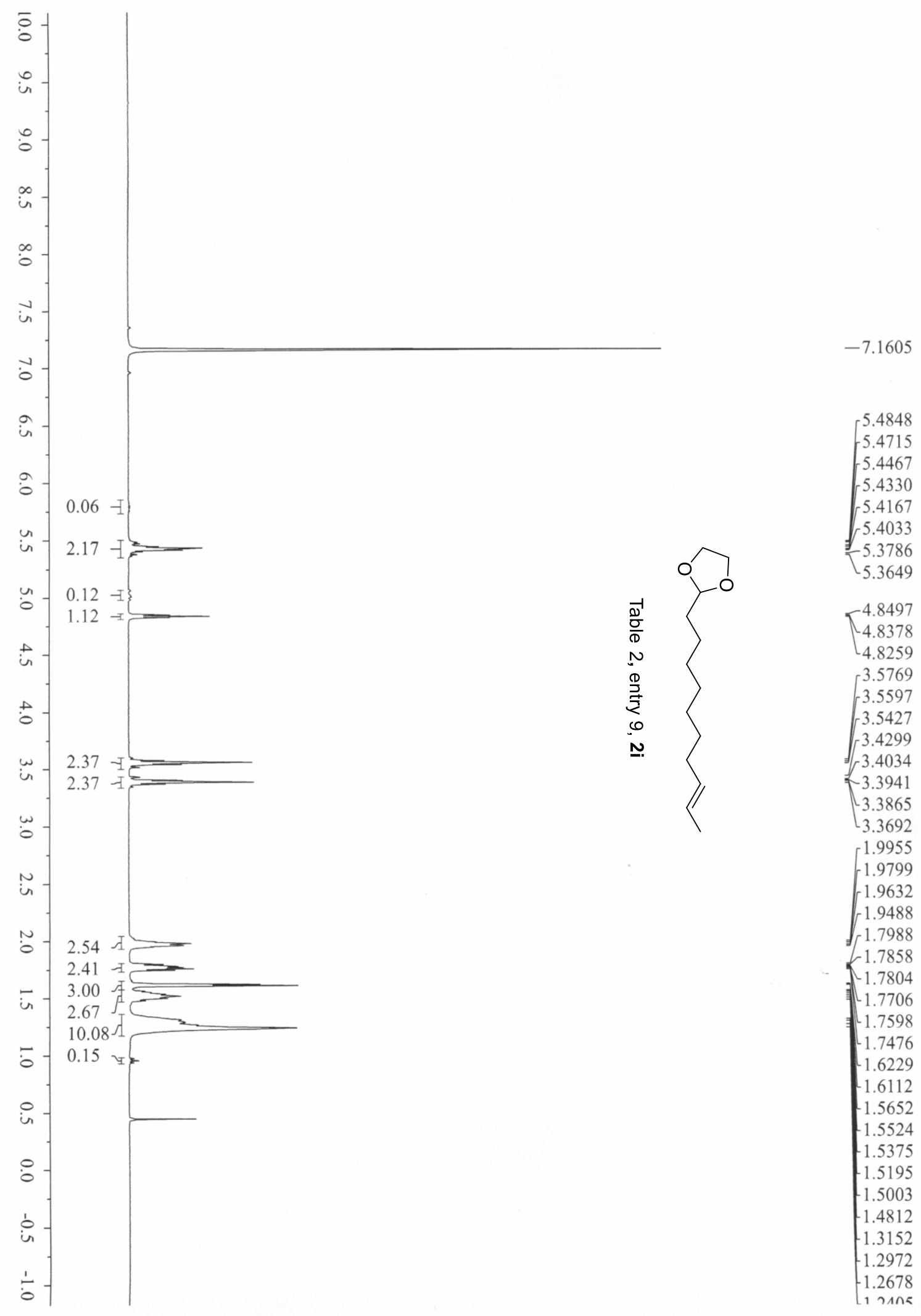




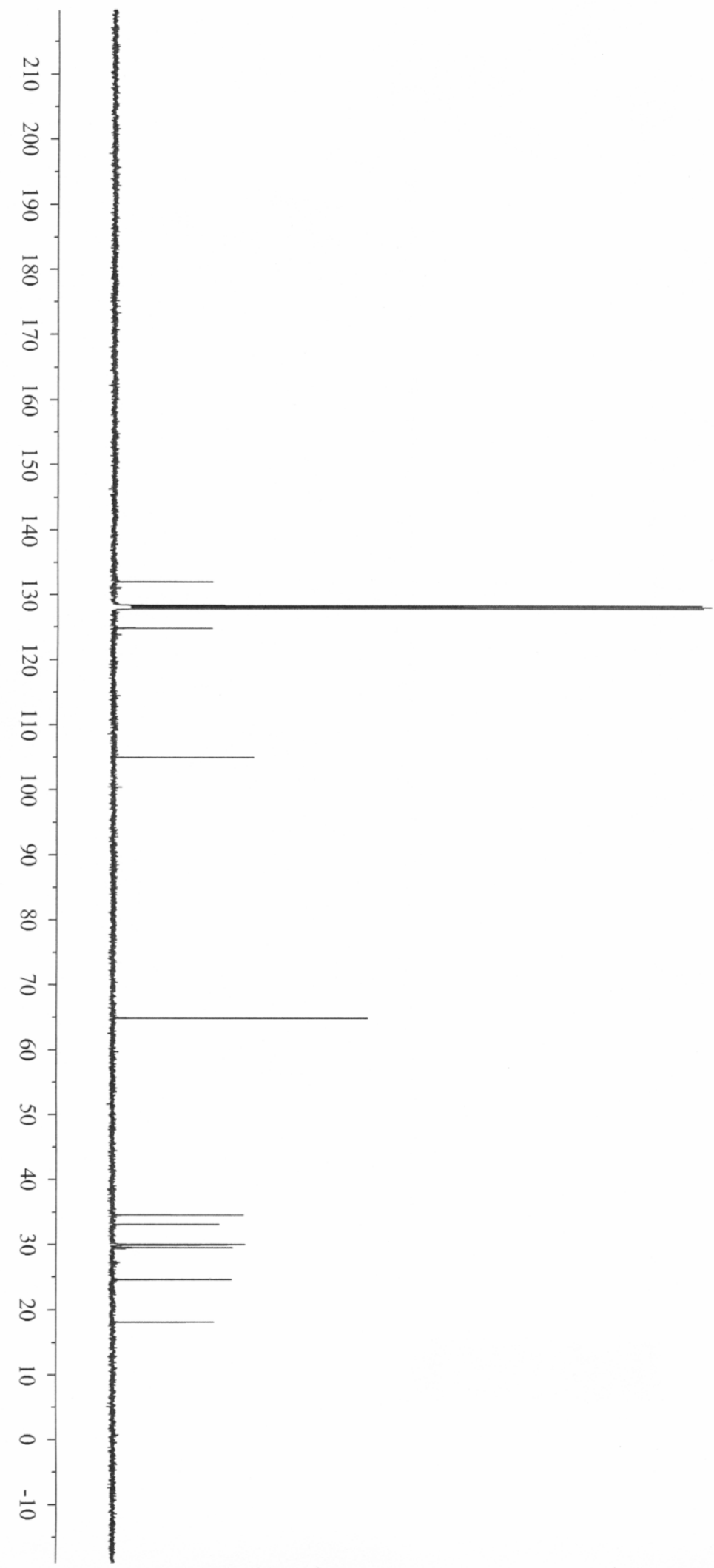

132.0023

128.3010

$-128.0604$

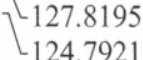

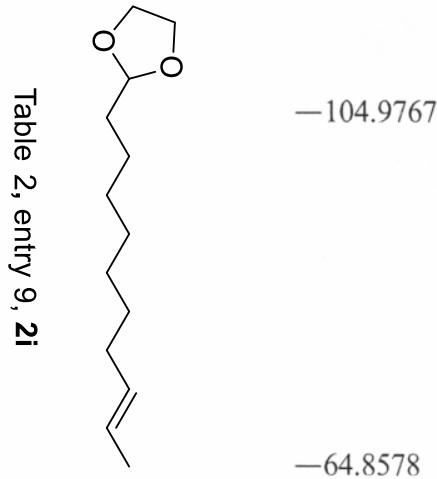

$-64.8578$

34.5598

33.0930

30.0245

29.9809

29.9083

$-29.5097$

-24.5856

18.1421 


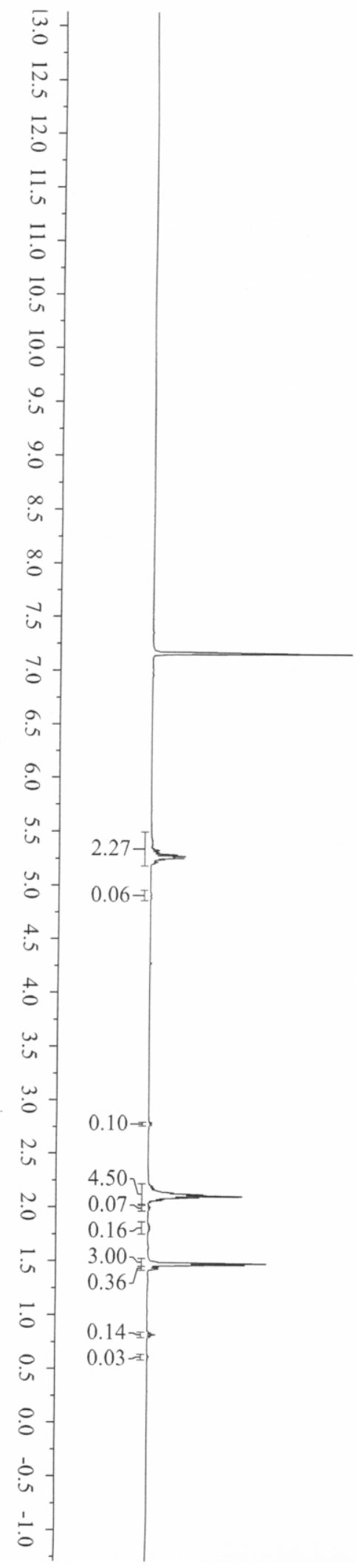

$-7.1601$

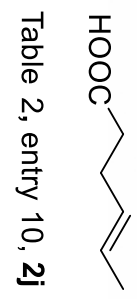

$\left\{\begin{array}{l}5.4665 \\ 5.4176 \\ 5.3531 \\ 5.3271 \\ 5.2887 \\ 5.2750 \\ 5.2568 \\ 5.2430 \\ 5.2042\end{array}\right.$

2.2129

$-2.1941$

$-2.1364$

2.1210

2.1043

2.0930

2.0669

2.0466

1.4808

$-1.4678$

1.4463

1.4294 


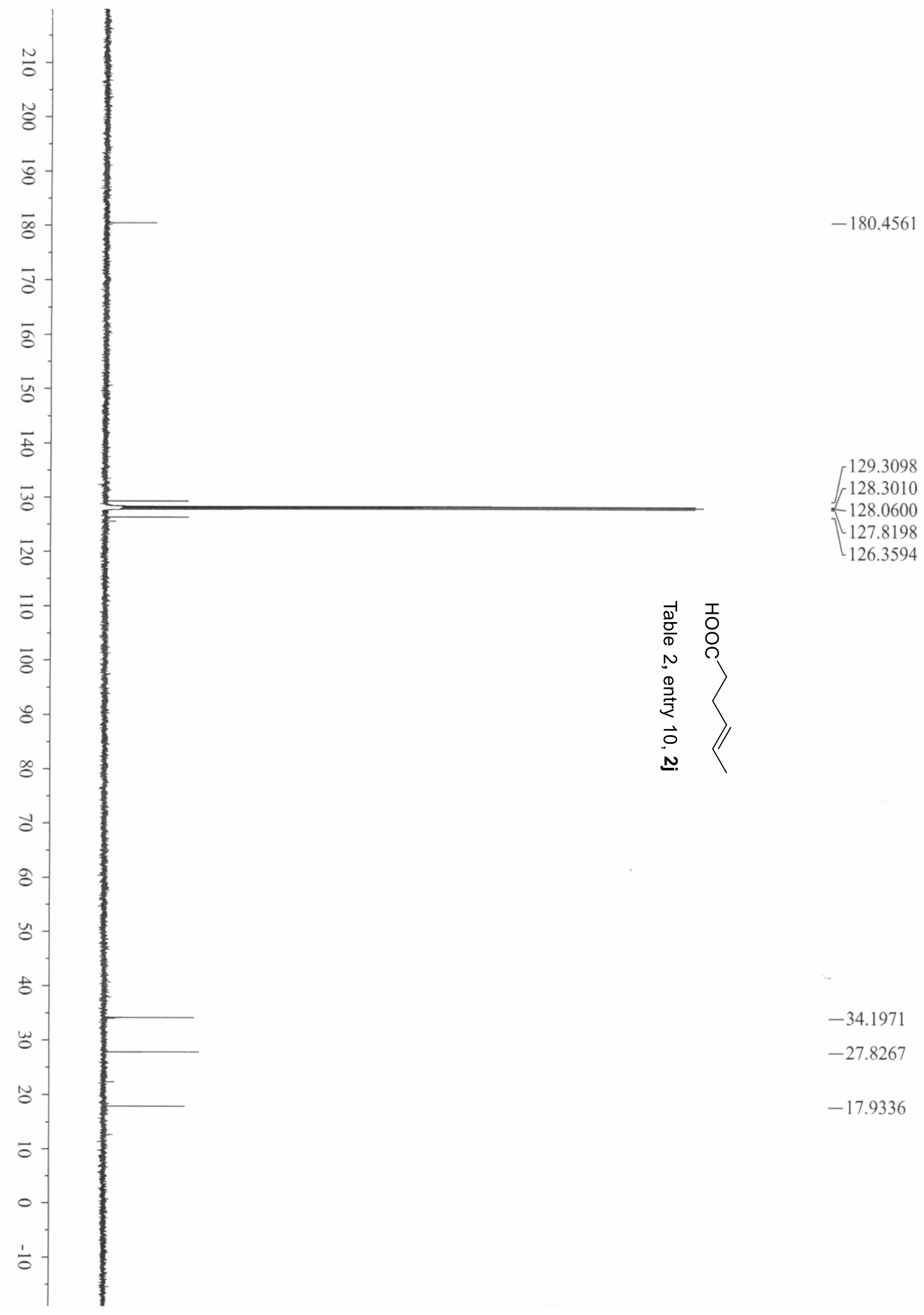



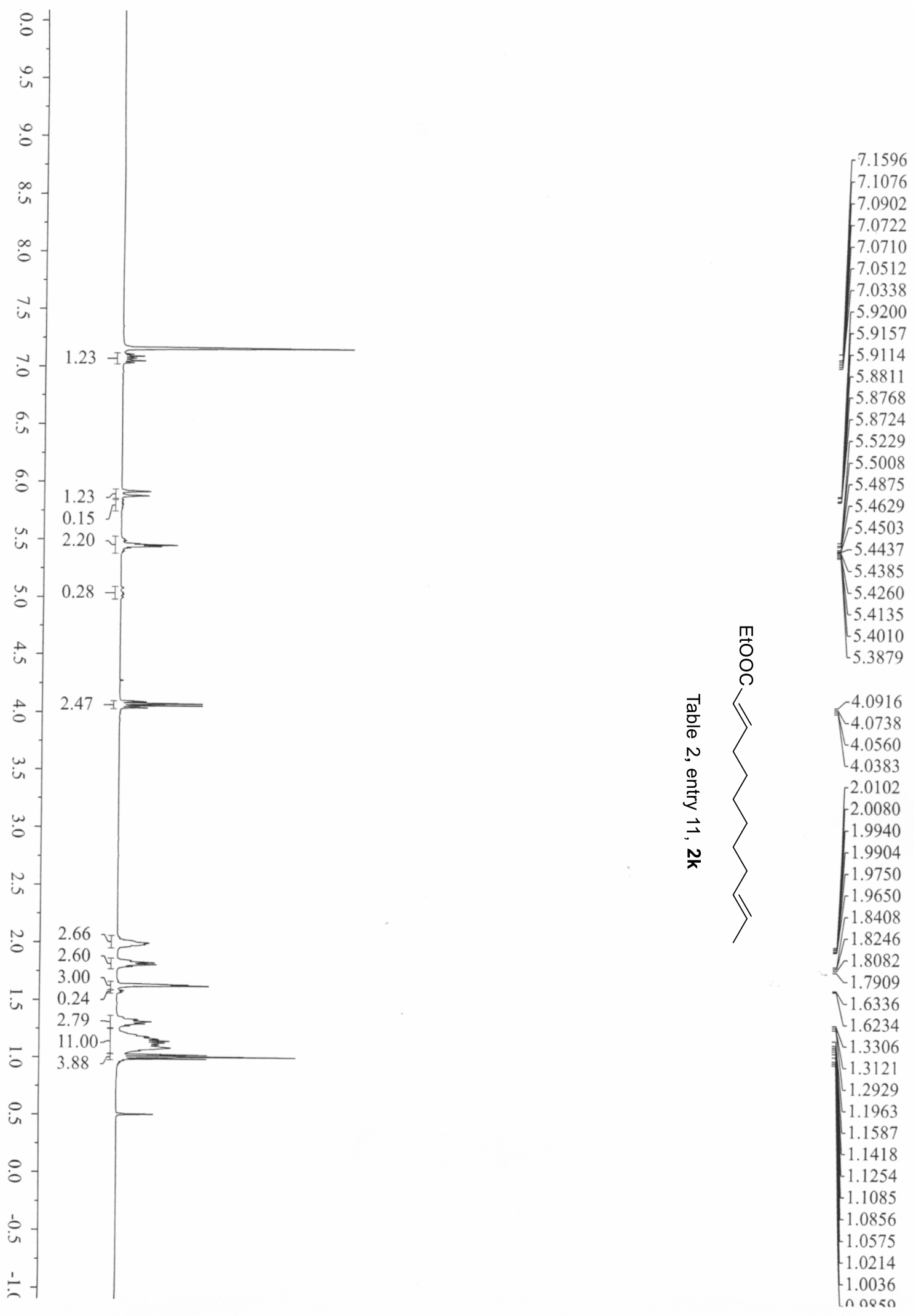


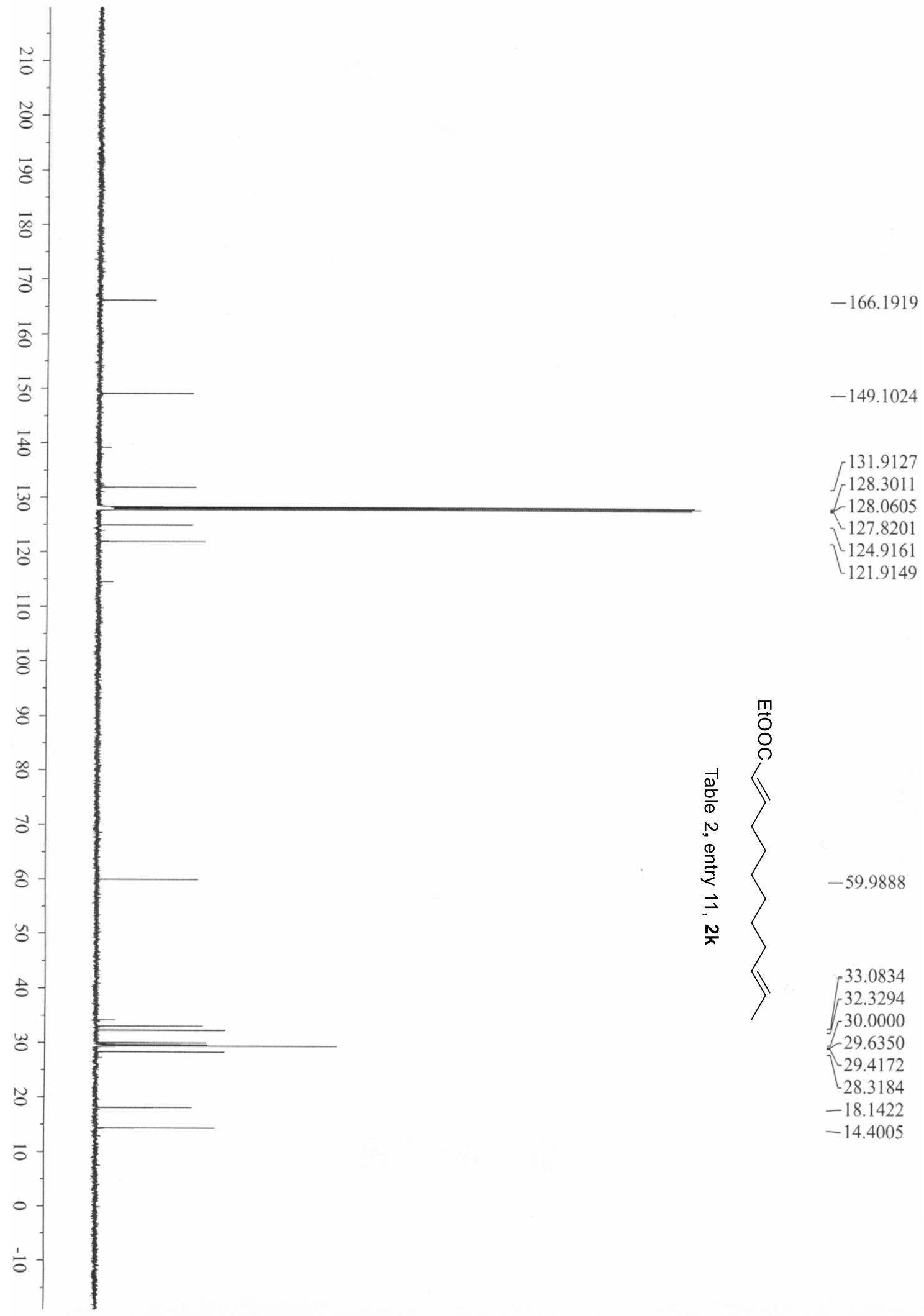




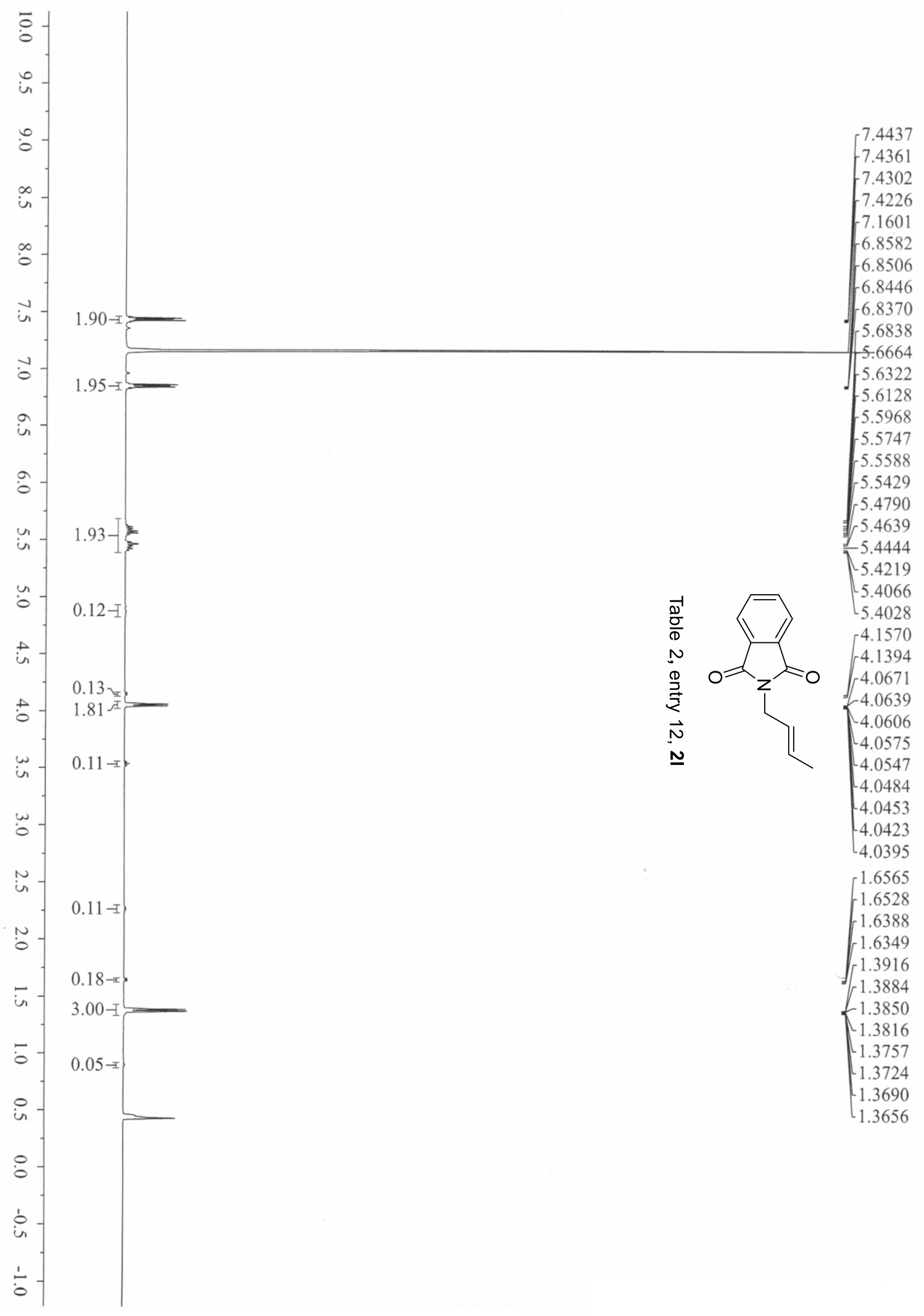




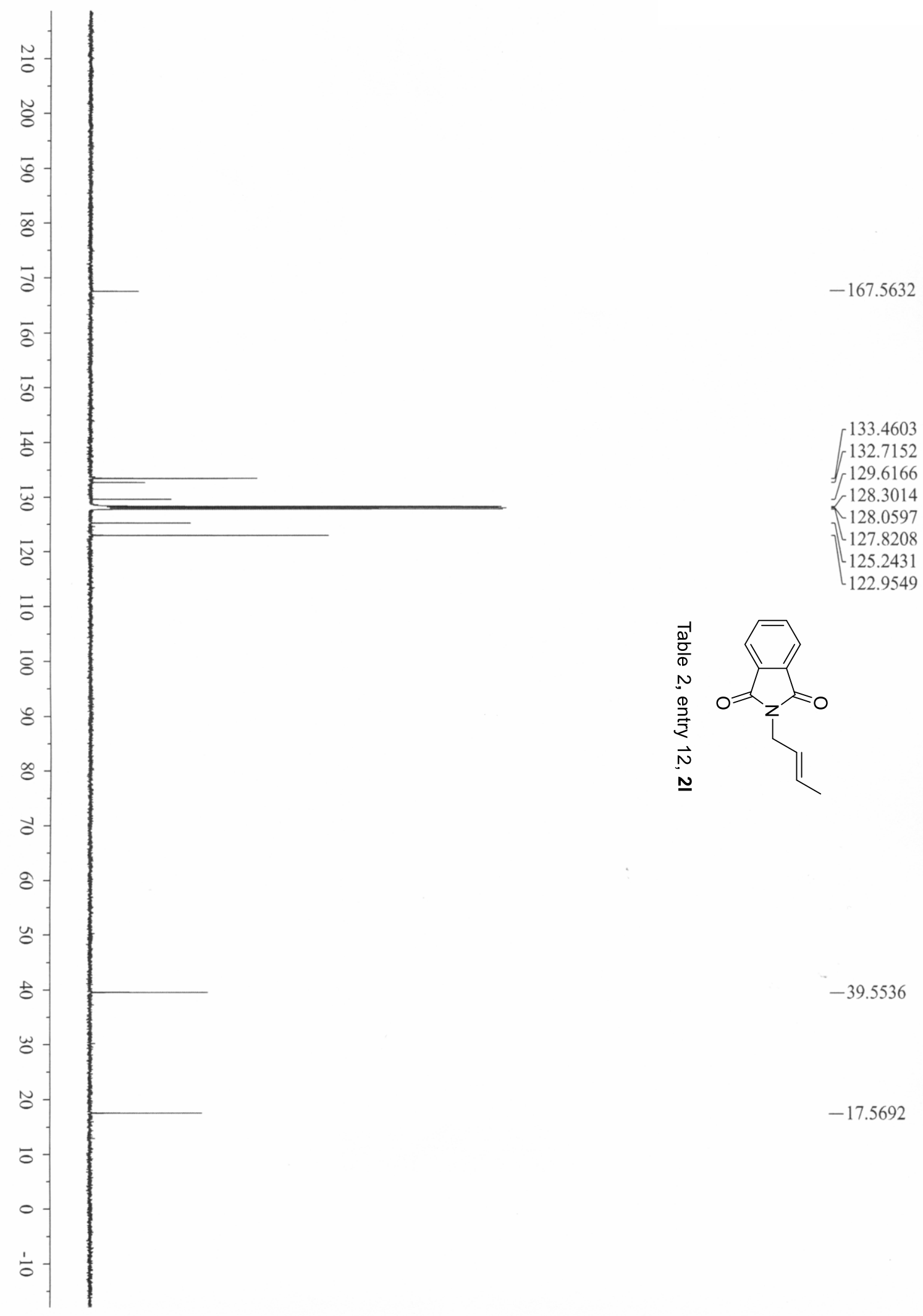




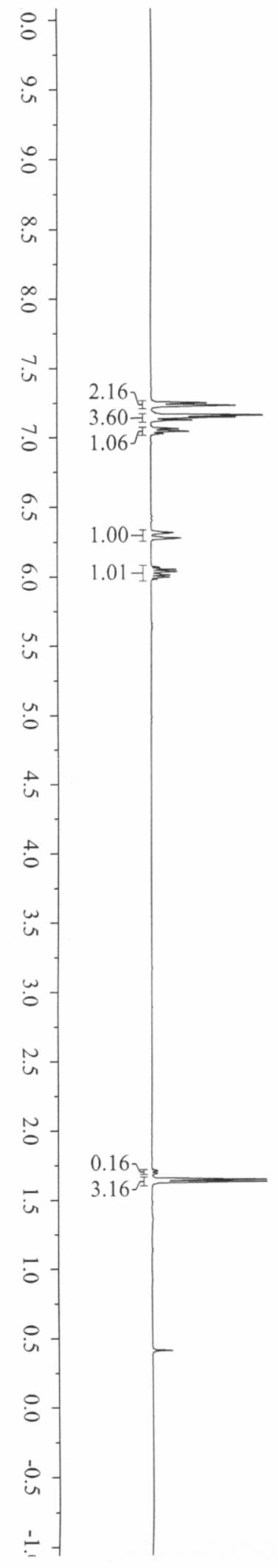

7.2490

7.2304

7.1602

$-7.1442$

7.1249

7.0621

7.0440

7.0257

6.3167

6.2773

6.0691

6.0526

$-6.0360$

6.0300

$-6.0192$

$-6.0133$

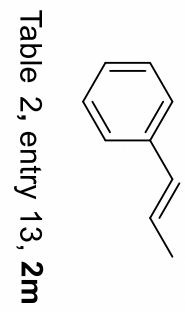

$-5.9967$

5.9802

1.7140

1.7099

1.6961

1.6920

1.6501

1.6467

1.6335

1.6301 


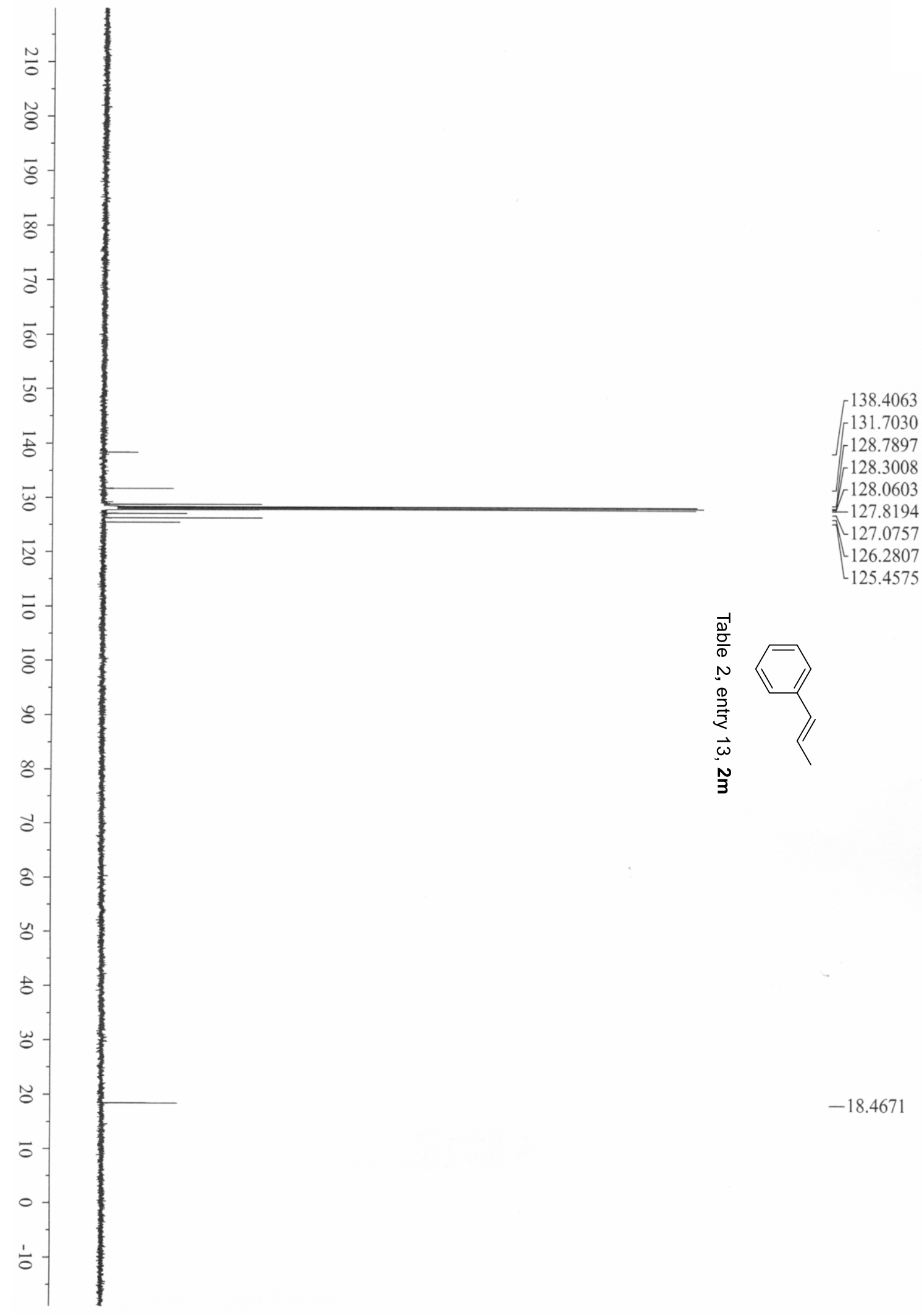




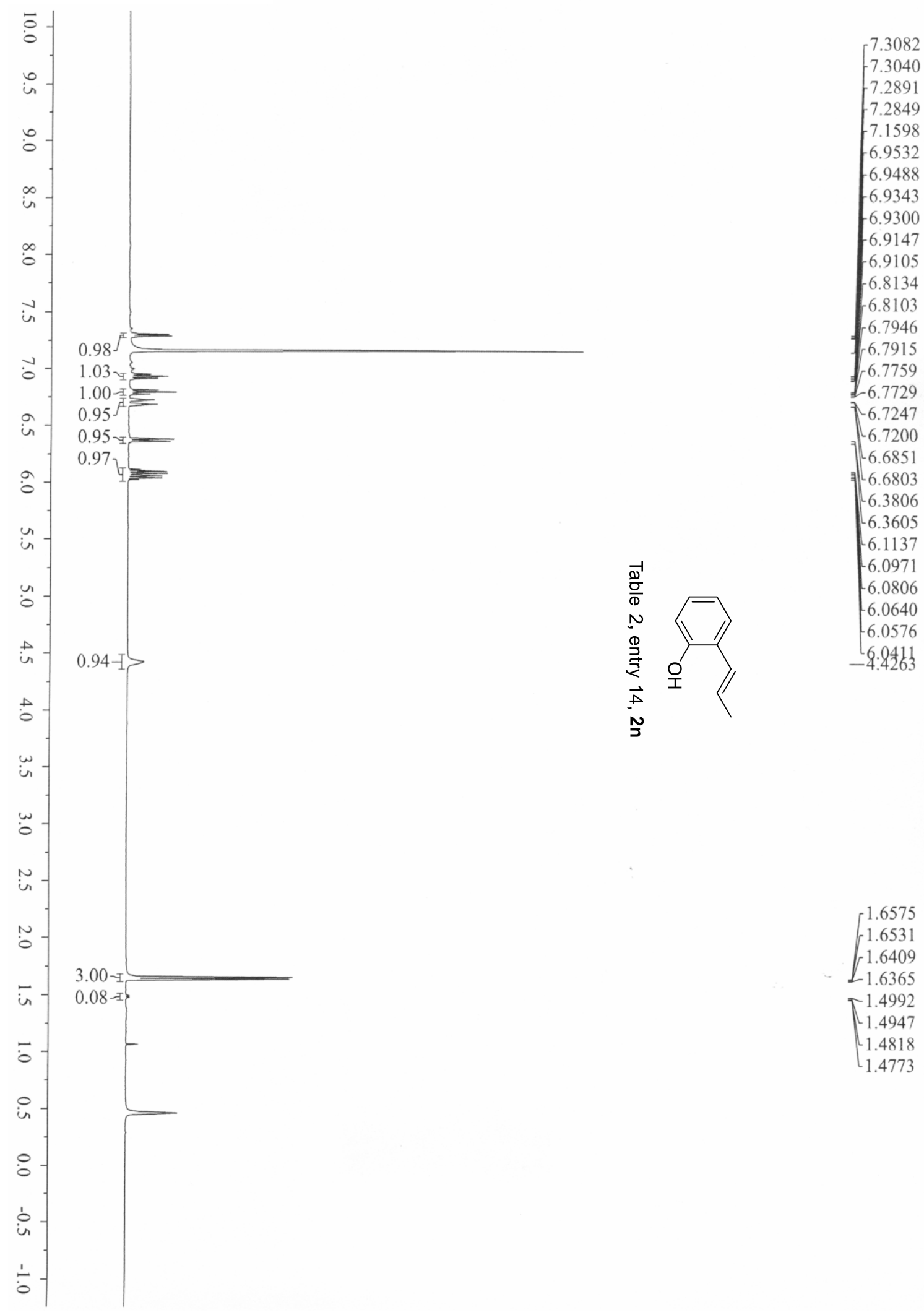




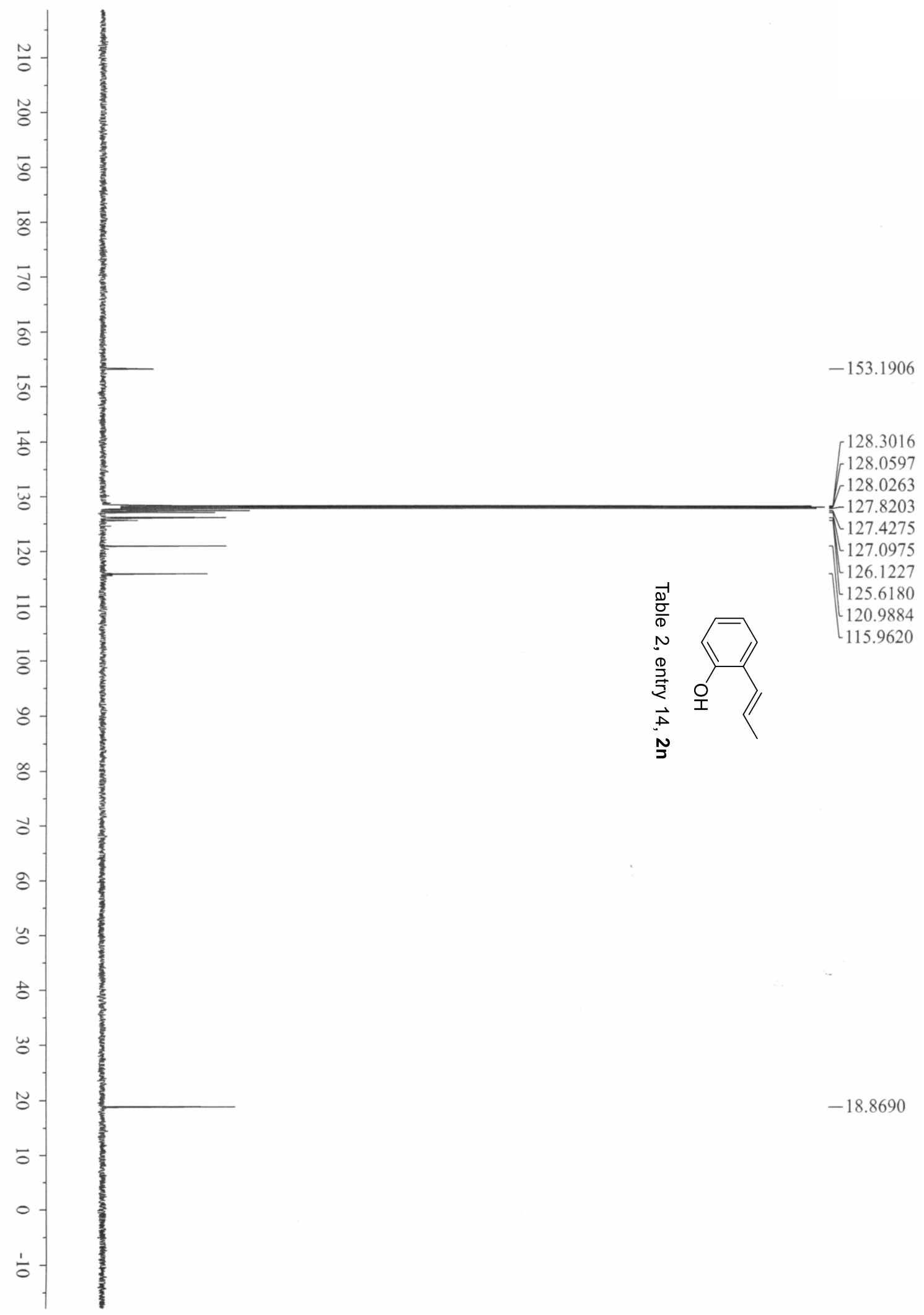




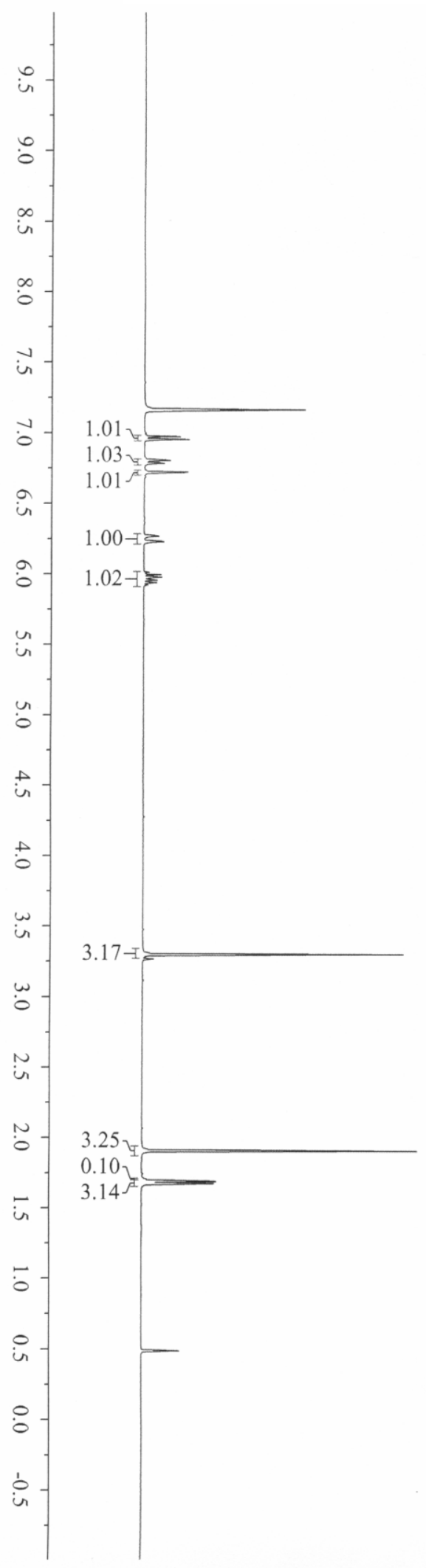




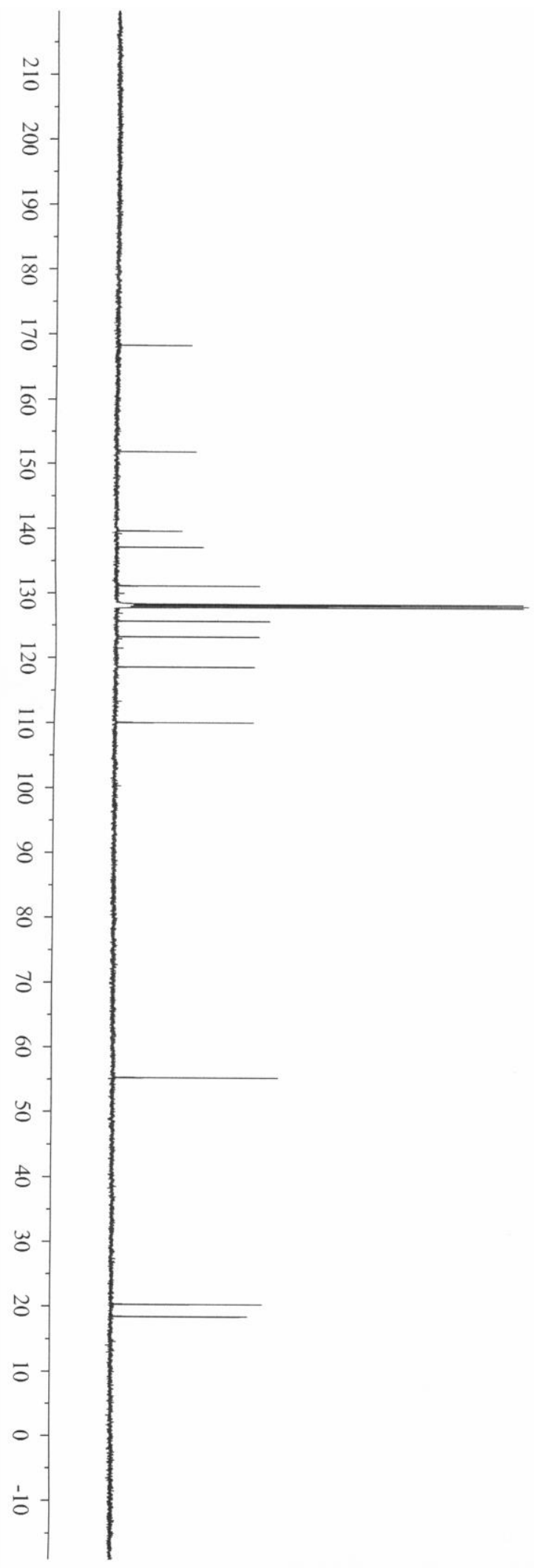

$-168.2798$

$-151.8481$

139.6455

137.1375

131.1426

128.3010

128.0602

$<_{127.8195}$

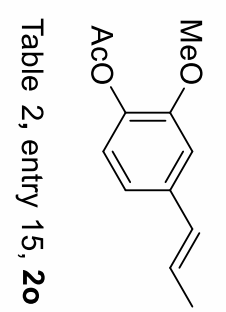

125.6492

$-123.2304$

118.5882

$-110.0744$

$-55.2756$

$-20.3123$

$\sim 18.4357$ 

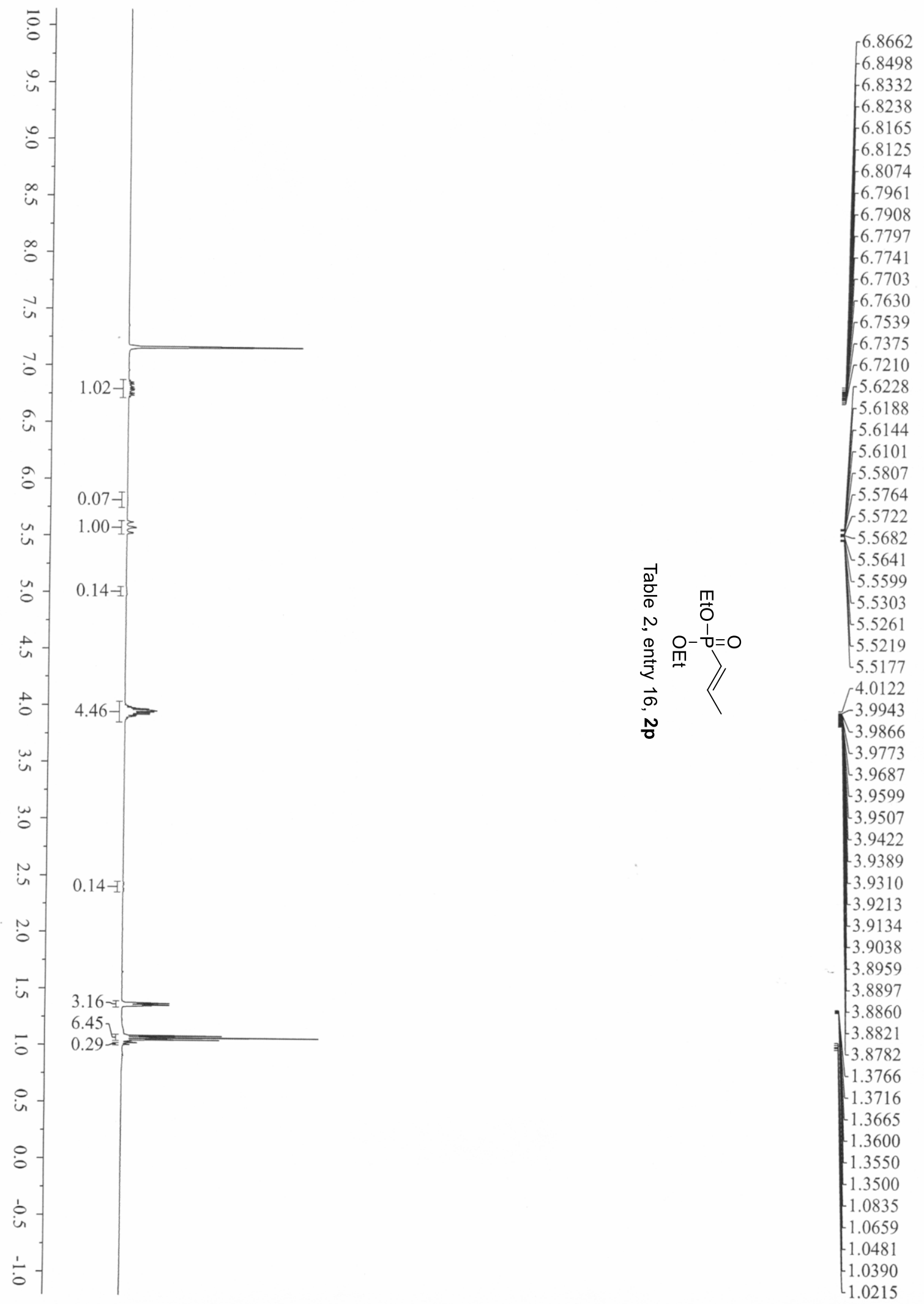


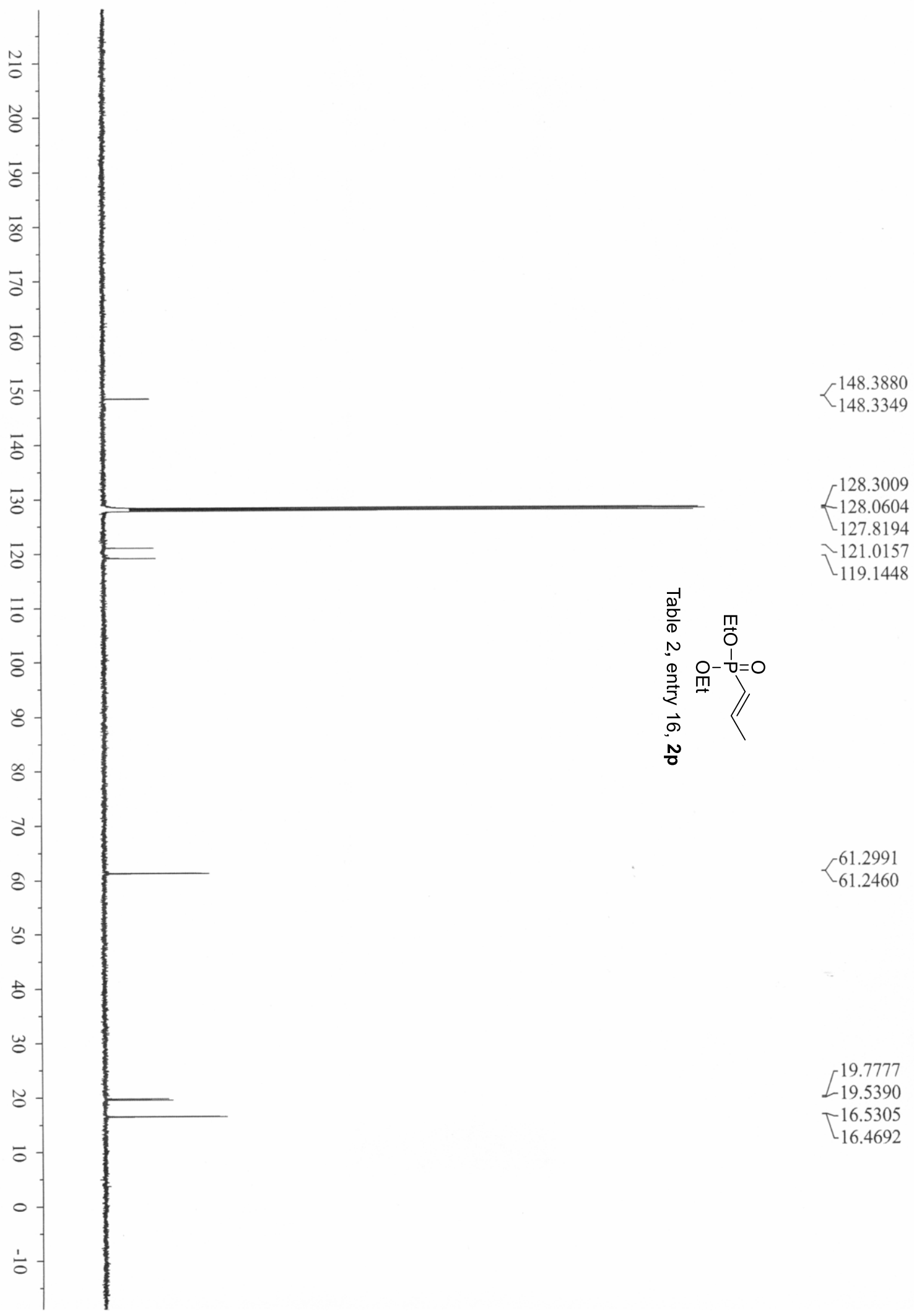




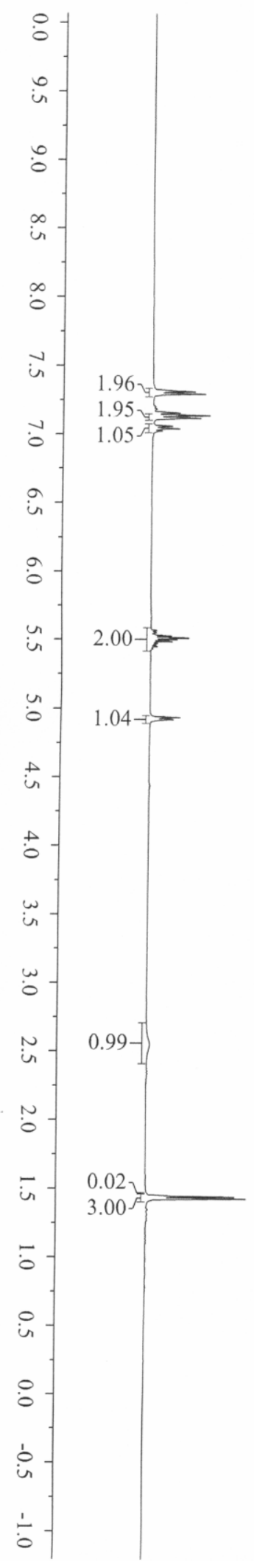




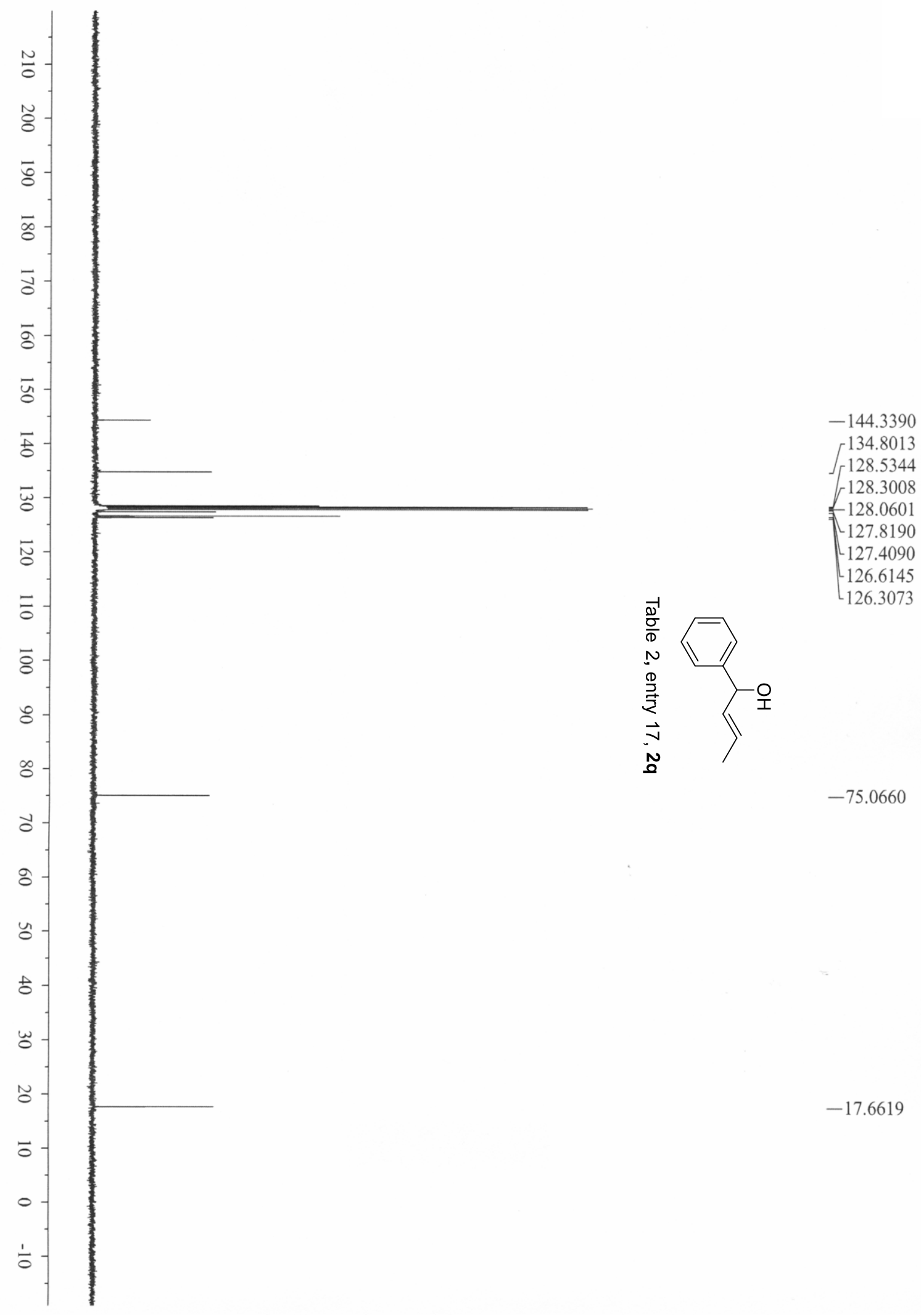



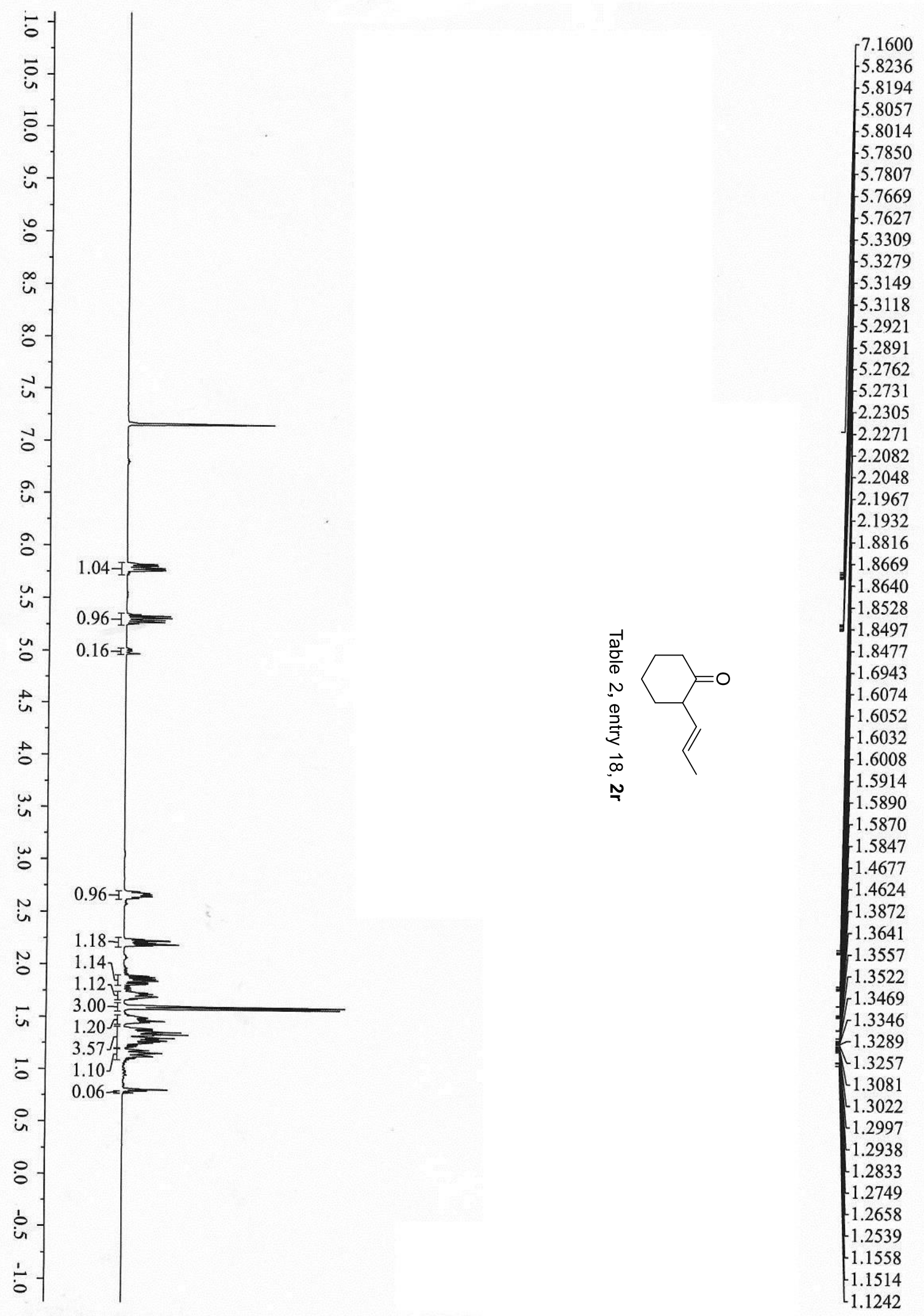


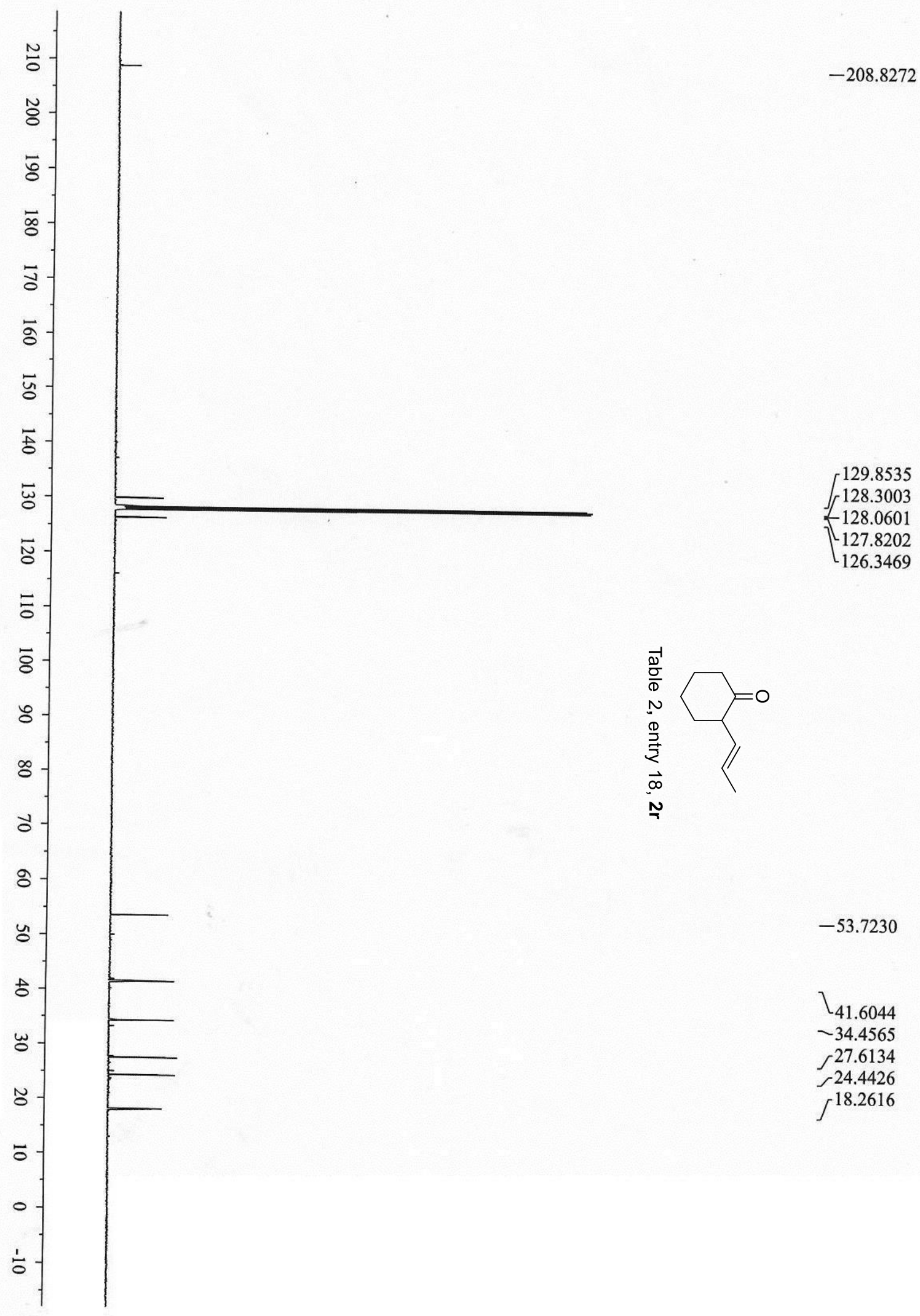




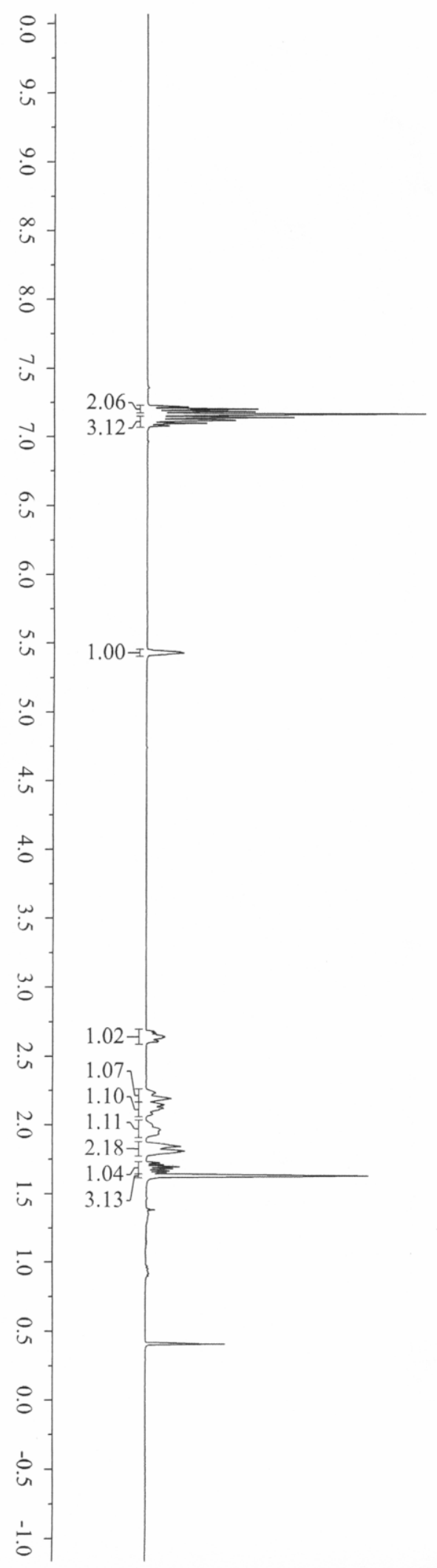



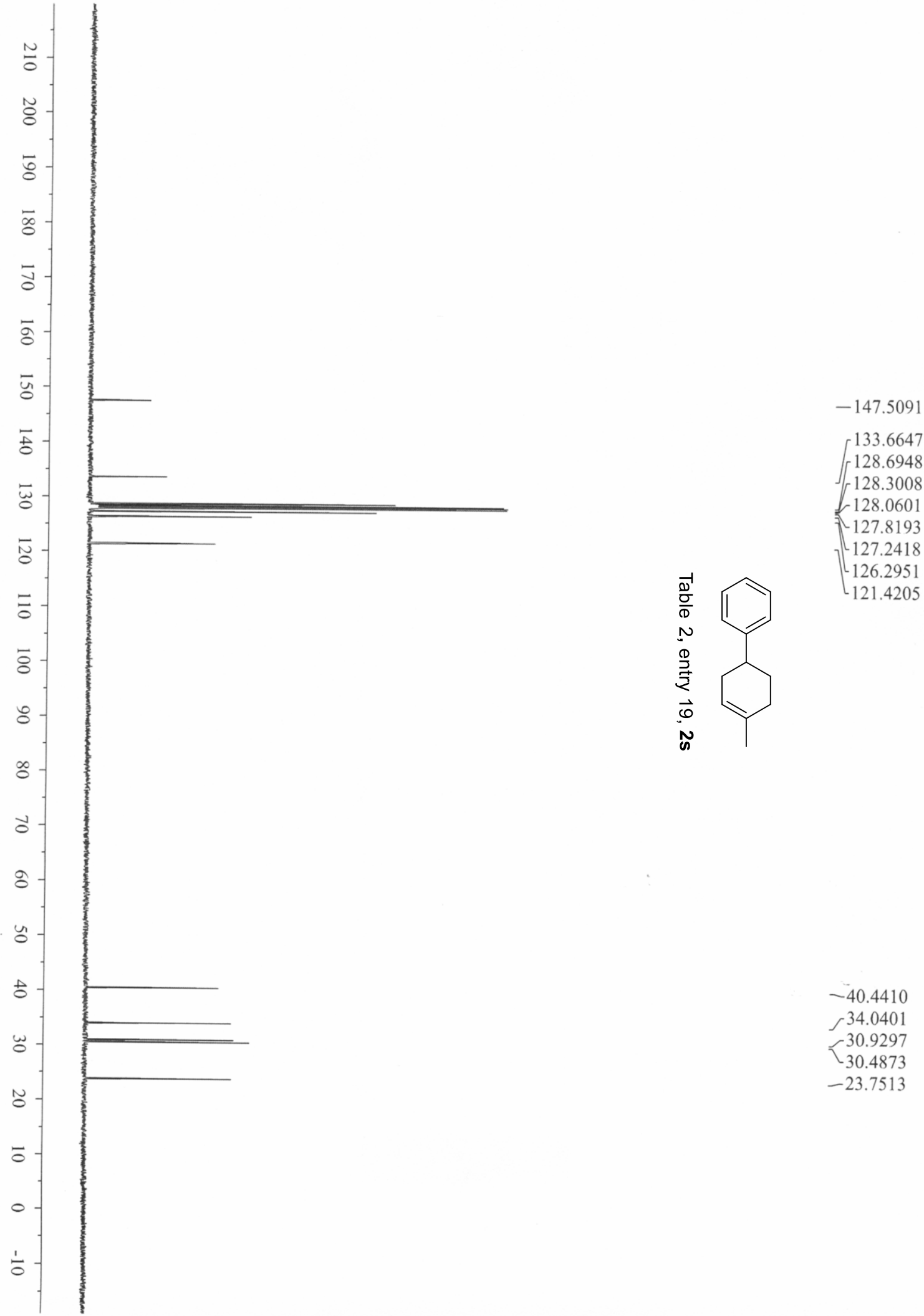

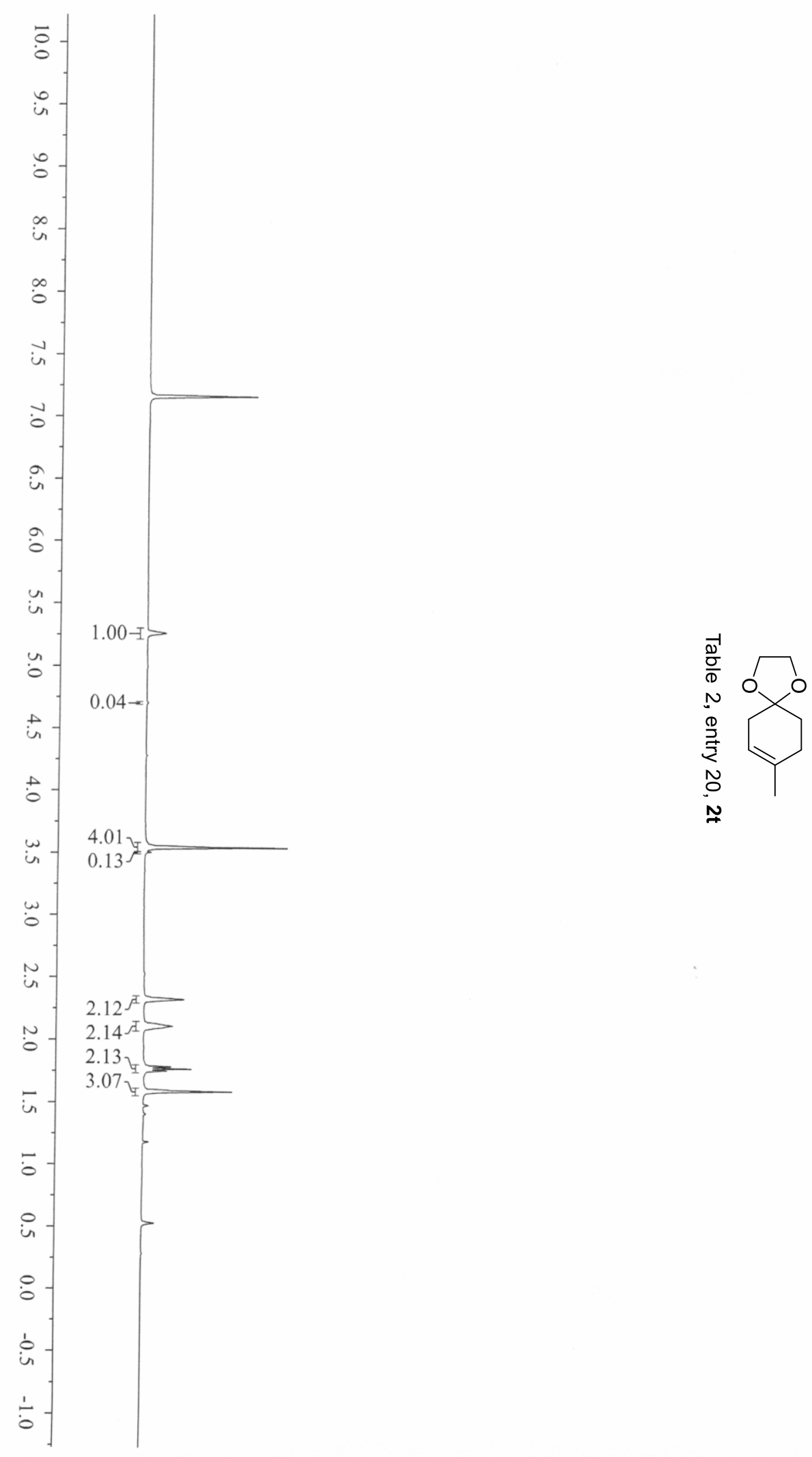

1.7665

1.7499

1.5874 


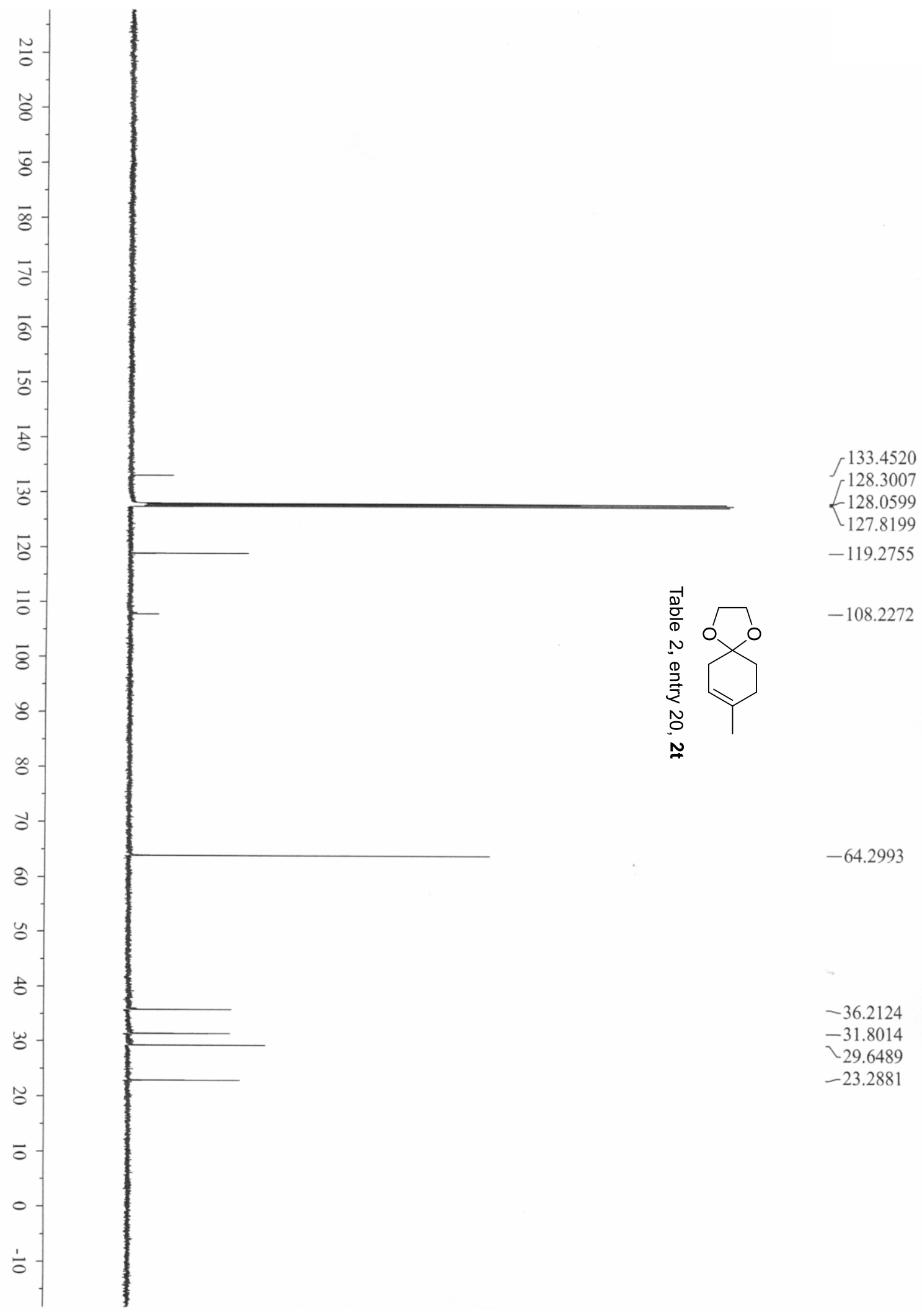




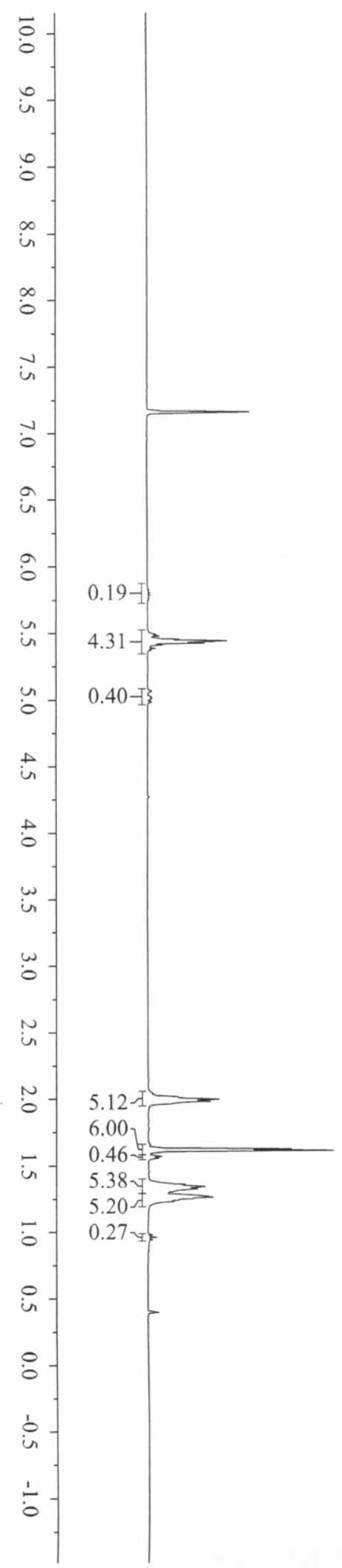




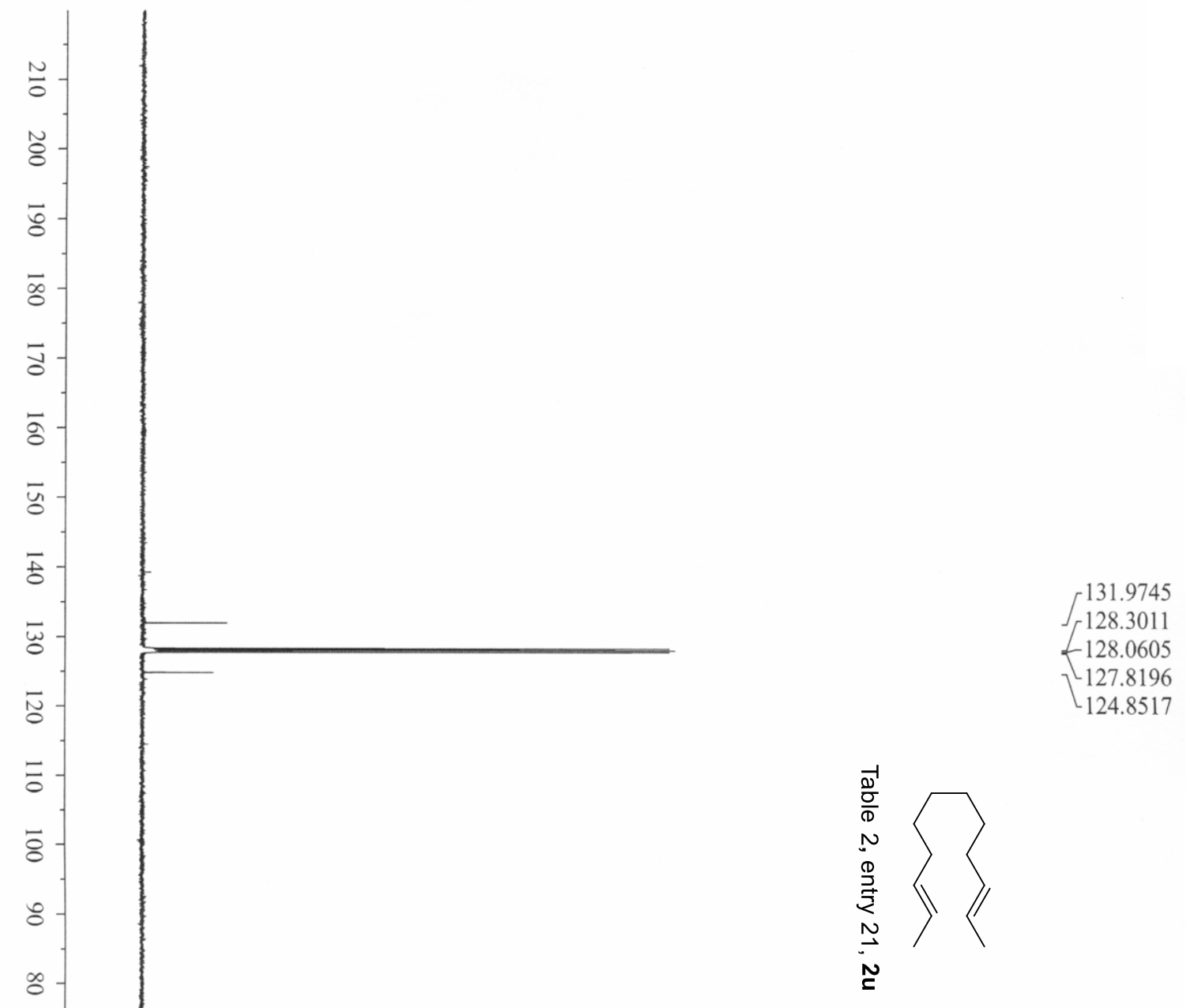

33.1134

$-30.0461$

29.5073

$-18.1454$ 


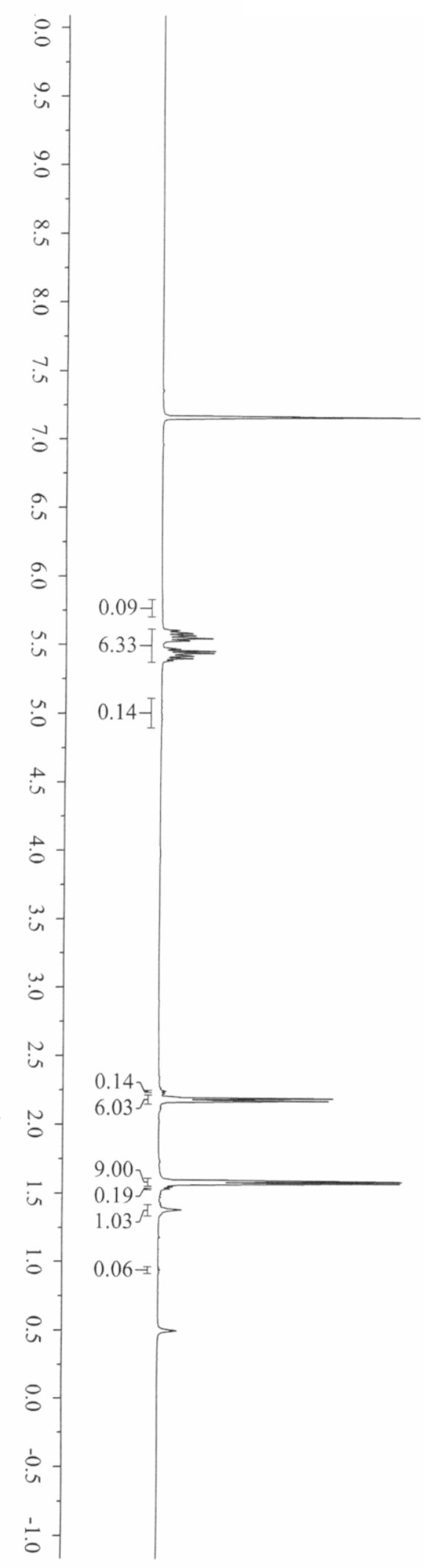




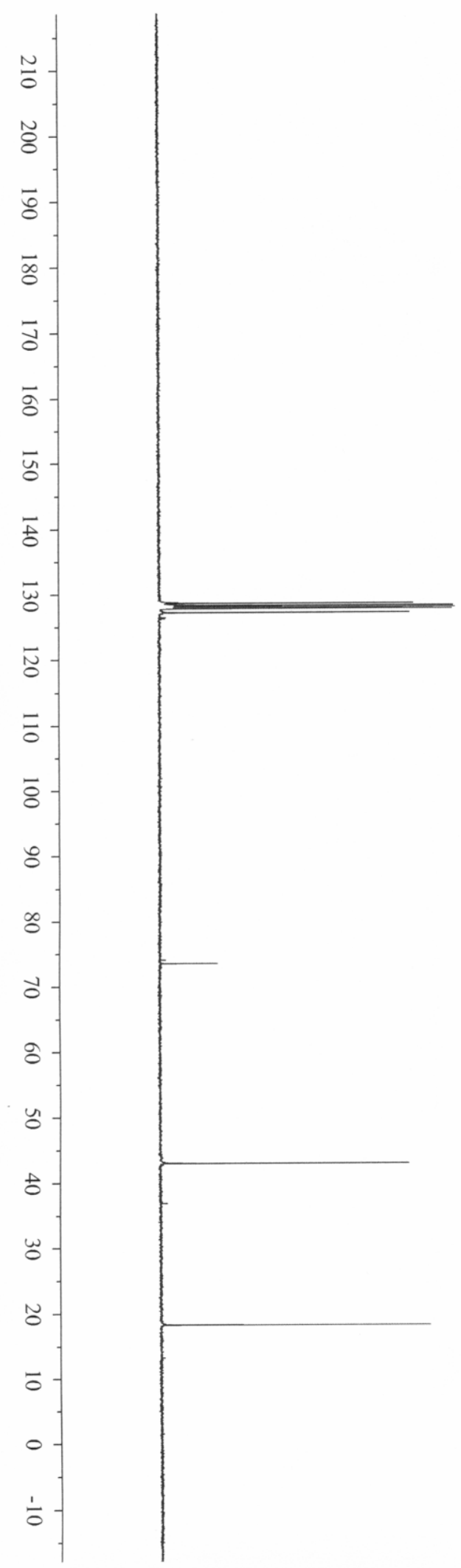

128.6484

128.3020

$-128.0601$

127.8197

127.2157

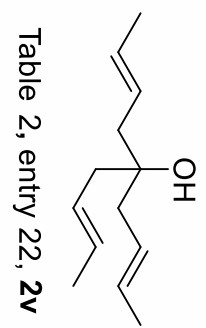

$-73.5422$

$-43.0002$

$-18.2776$ 


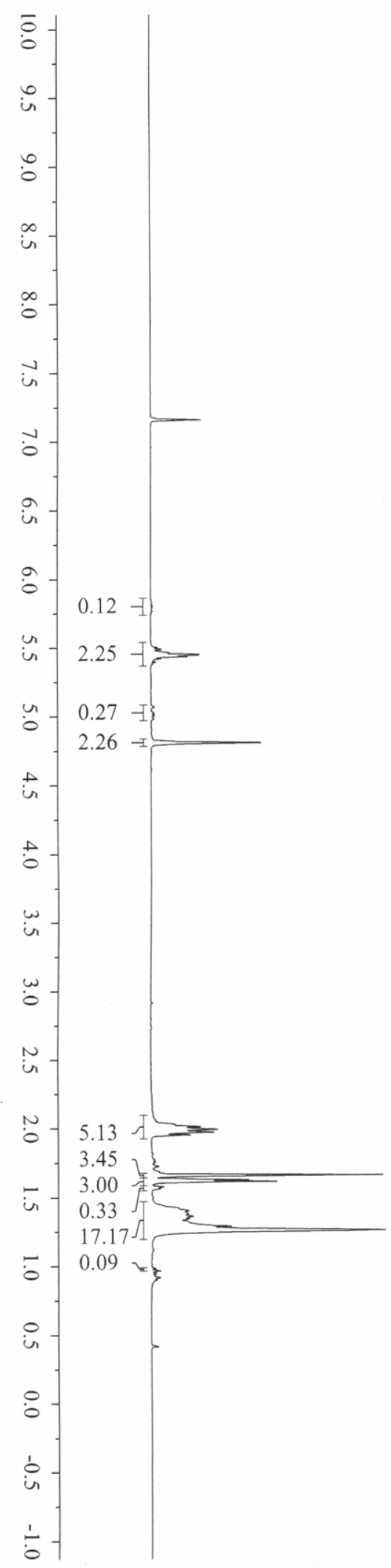

$-7.1600$ 5.8519 $-5.8353$ $-5.8096$ $-5.7926$ 5.7671 5.7503 5.5266 5.5010 5.4877 5.4511

$-5.4336$ 5.4192

$-5.3949$ $-5.3809$ $-5.0699$ $-5.0264$ $-5.0093$ $-4.9843$ 4.8097

2.0310

$-2.0140$ $-1.9938$ $-1.9734$ 1.9535 1.6623 1.6298 政 1.6179 1.5786 - 1.5663 $-1.4428$ 1.4066 1.3631 1.3027 1.2917 1.2611 $-0.9852$ $-0.9667$ $-0.9480$ $-0.9327$ $-0.9176$ 0.9014 


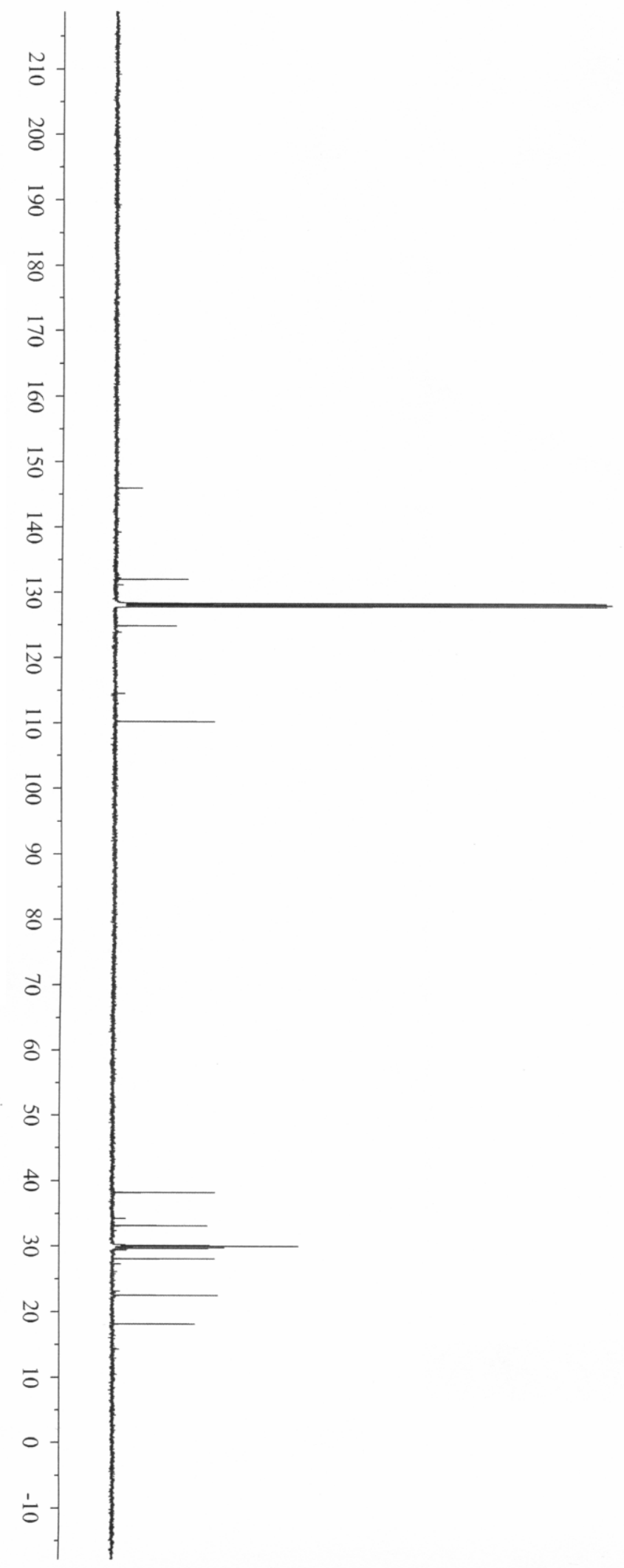

$-145.9526$

131.9936 128.3001

$-128.0597$

127.8205

124.8726

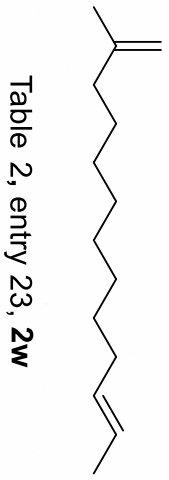

$-110.2456$

38.2327

33.1520

30.1001

29.9955

29.7615

29.6566

28.0732

22.5139

18.1567 


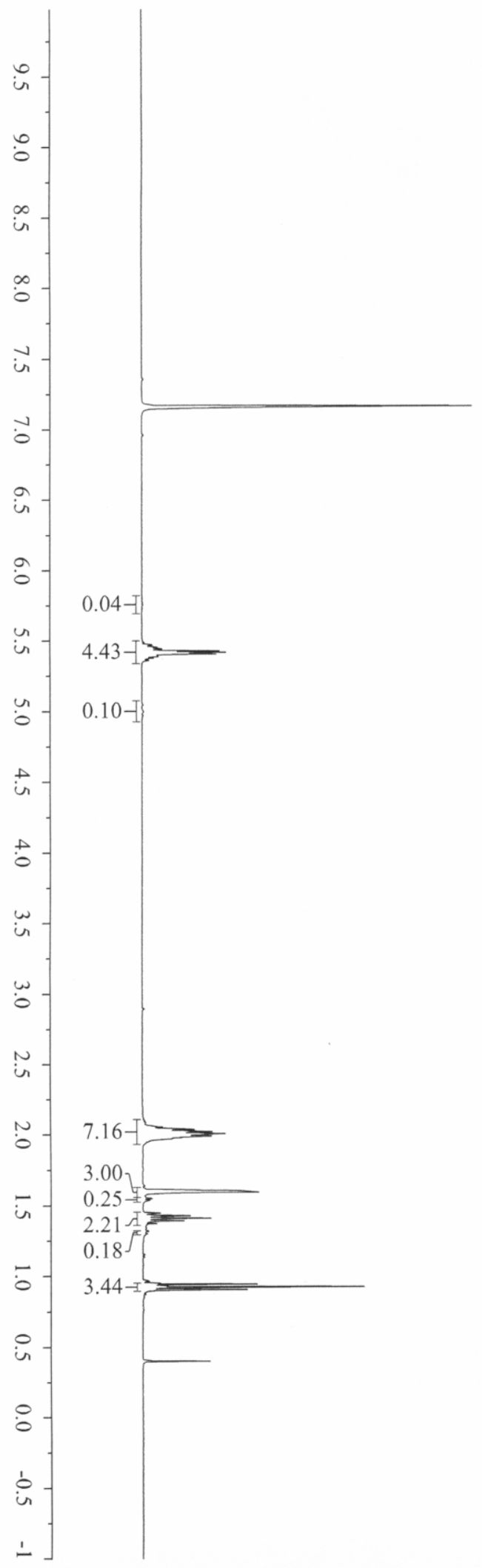




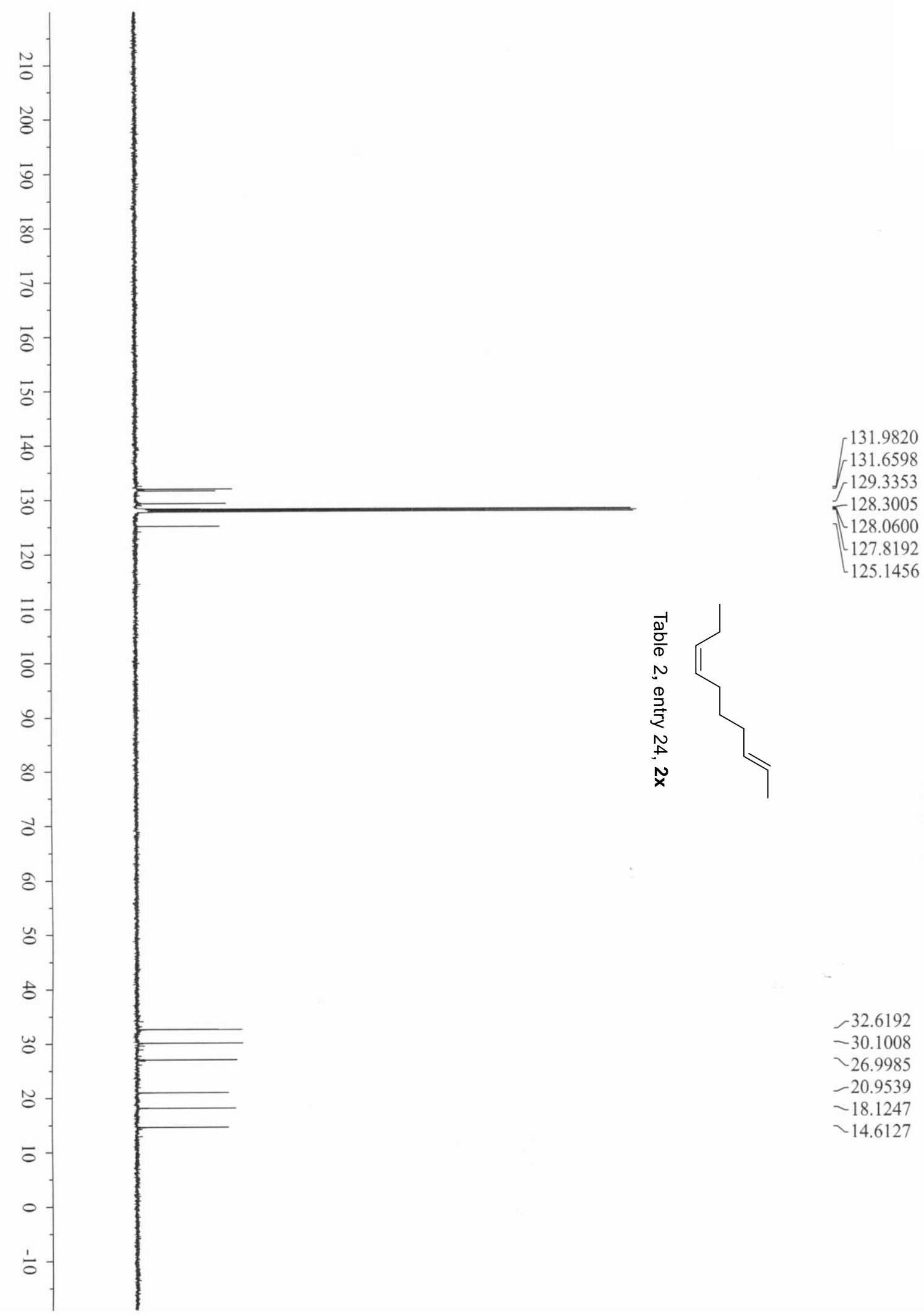



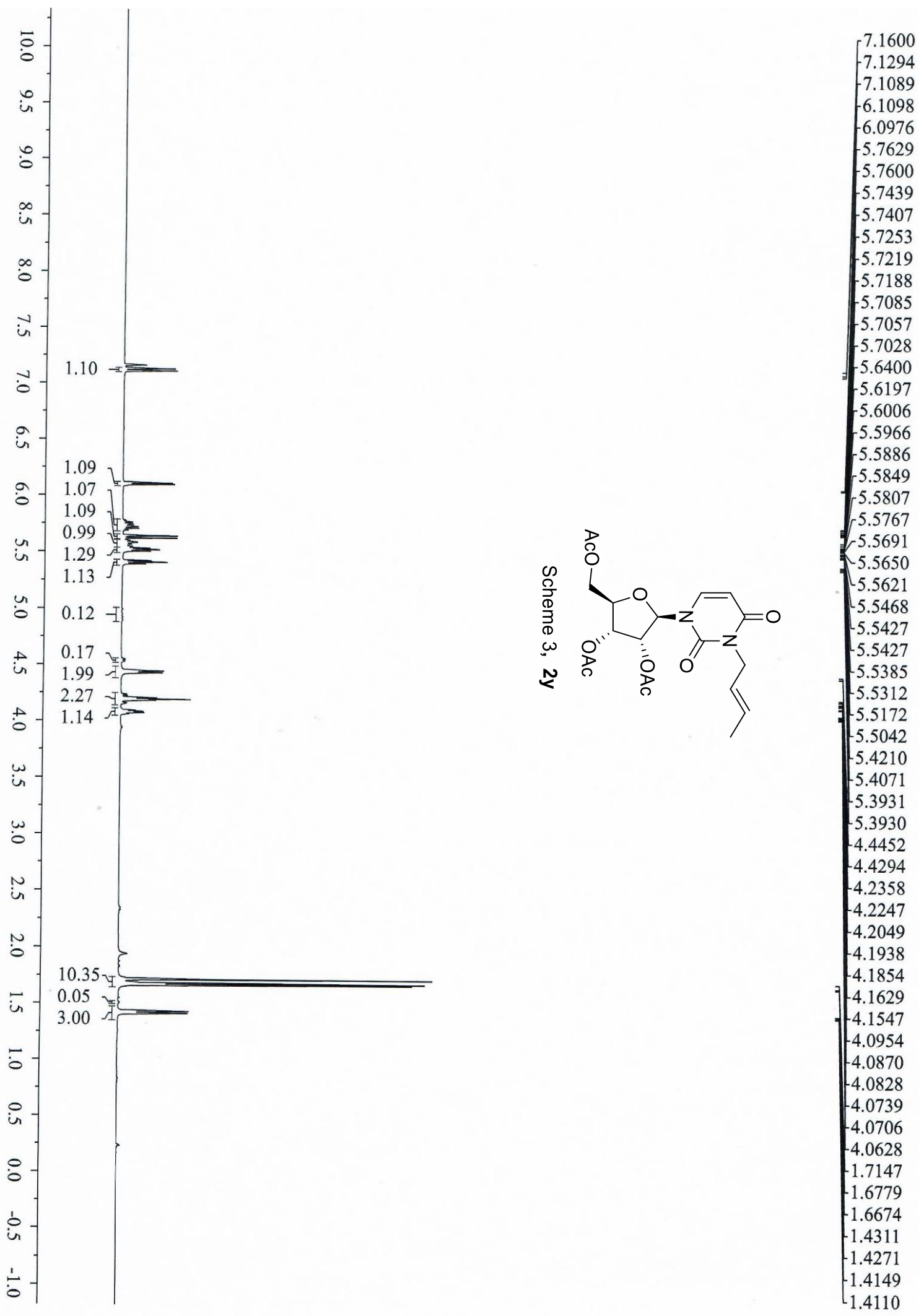


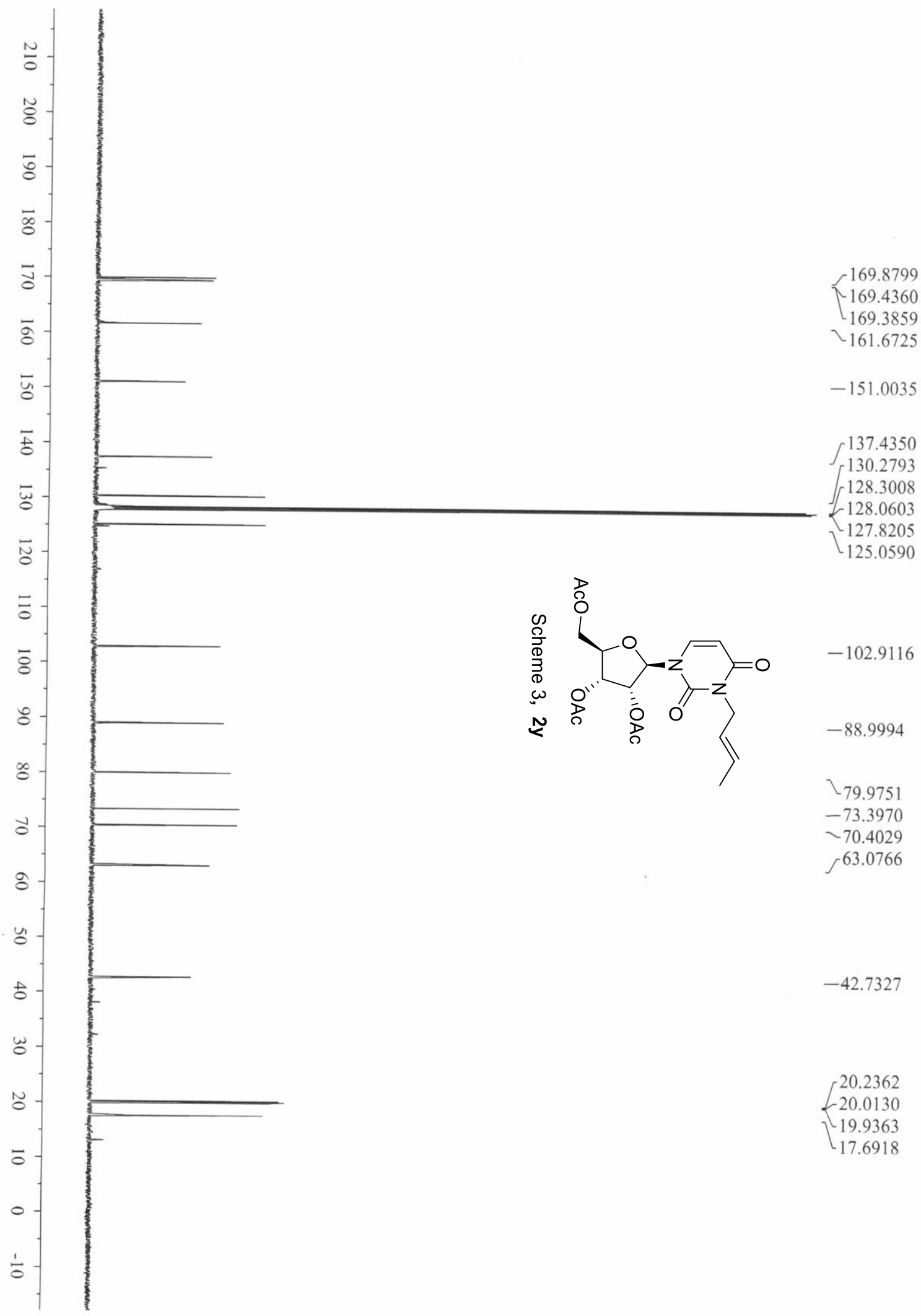




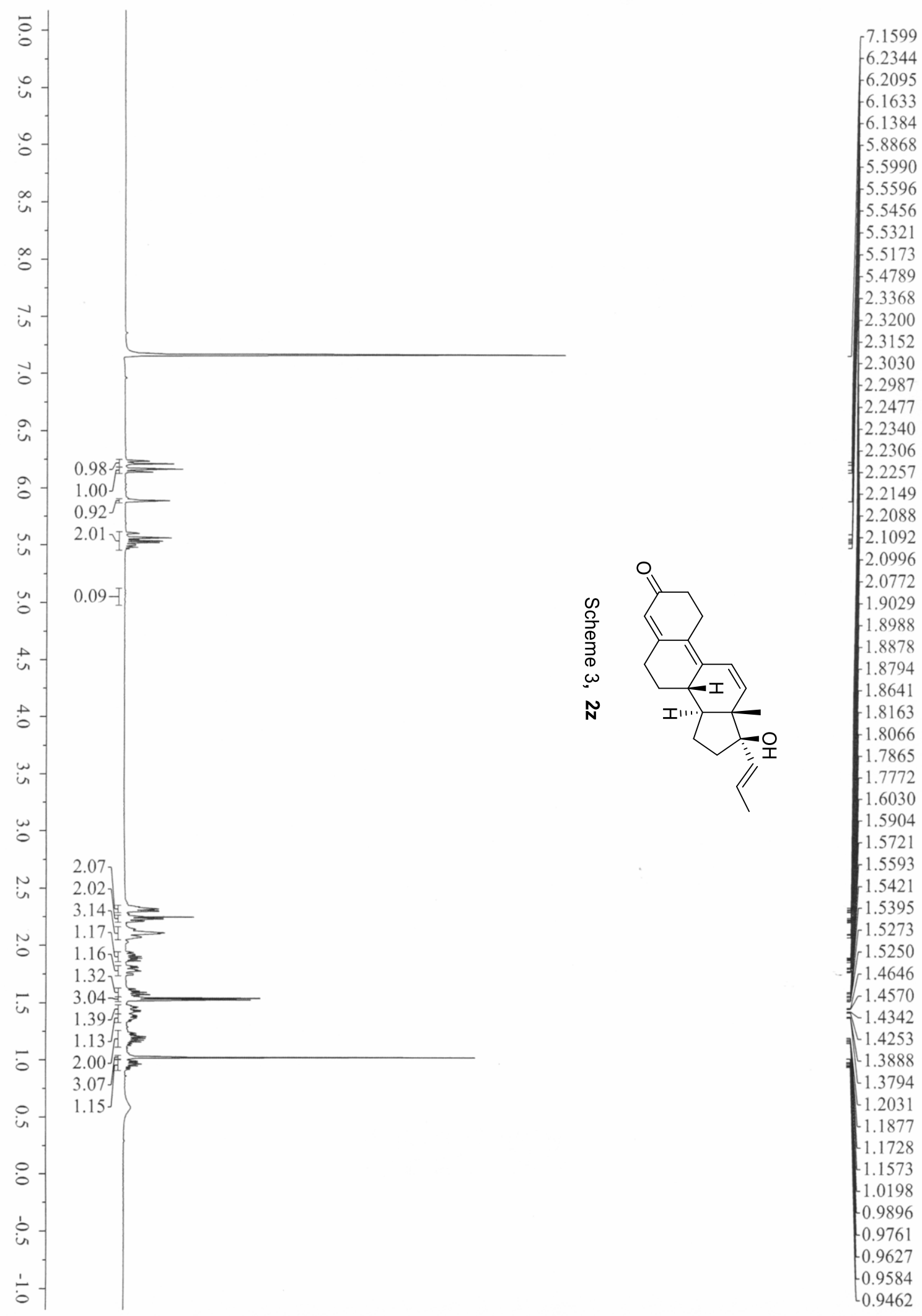




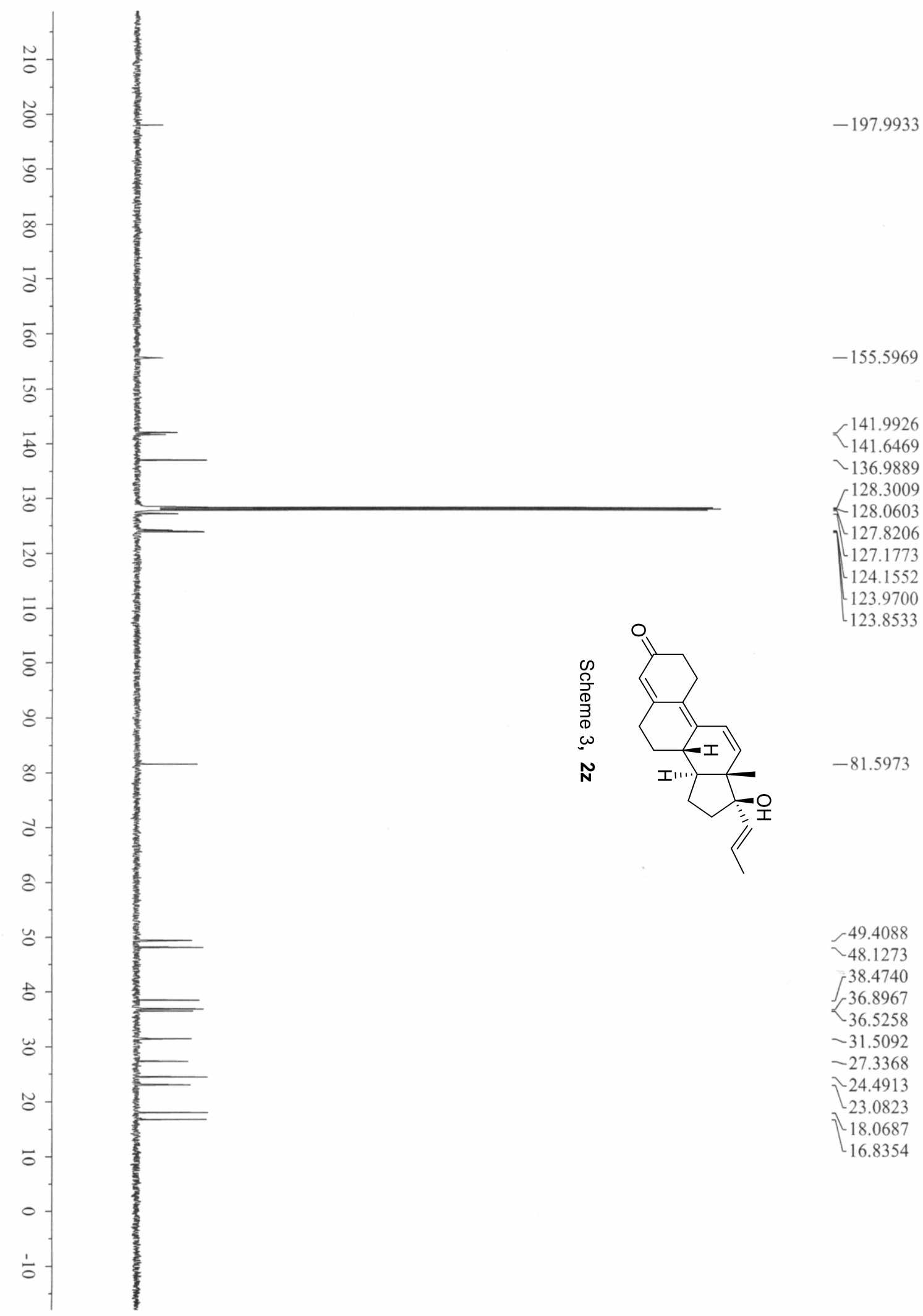

